\title{
Visible-light-promoted biomimetic reductive functionalization of quaternary benzophenanthridine alkaloids
}

Lin Wang a, ${ }^{\S}$, Xinhao Wang ${ }^{\text {a, }}$, Wei Wang ${ }^{\text {a }}$, Wei Liu ${ }^{\text {a, b }}$, Yisong Liu ${ }^{\text {a, b }}$, Hongqi Xie ${ }^{\text {a, b }}$, Oliver Reiser c, Jianguo Zeng a,b*, Pi Cheng a,b*

a Hunan Key Laboratory of Traditional Chinese Veterinary Medicine, Hunan Agricultural University, Changsha, Hunan 410128, China

b College of Veterinary Medicine, Hunan Agricultural University, Changsha, Hunan 410128, China

c Institut für Organische Chemie, Universität Regensburg, Universitätsstr. 31, 93053 Regensburg, Germany

*corresponding authors: picheng@hunau.edu.cn; picheng55@126.com (P.C.) zengjianguo@hunau.edu.cn (J. Z.)

$\S$ These authors contribute equally to this work. 
1. HPLC-HR-MS analysis of blue-light promoted reduction of sanguinarine by NADH..................................................................

2. Photoreactor and general procedure for photoreaction ....................................

3. HPLC-HR-MS analysis of blue-light promoted reduction of sanguinarine by DHP 6j 4

4. NMR spectra data of target compounds.....................................

5. NMR spectra of target compounds...................................... 15 


\section{Blue-light mediated reduction of sanguinarine by NADH}

A solution of sanguinarine $(0.2 \mathrm{mmol})$ and NADH $(0.2 \mathrm{mmol})$ in DMSO $(2 \mathrm{~mL})$ was stirred under the irradiation of $455 \mathrm{~nm}$ blue light. After 12 hours, the reaction solution was analyzed by Agilent 6530 HPLC-Q-TOF/MS system. Results was shown in Figure S1 and S2.
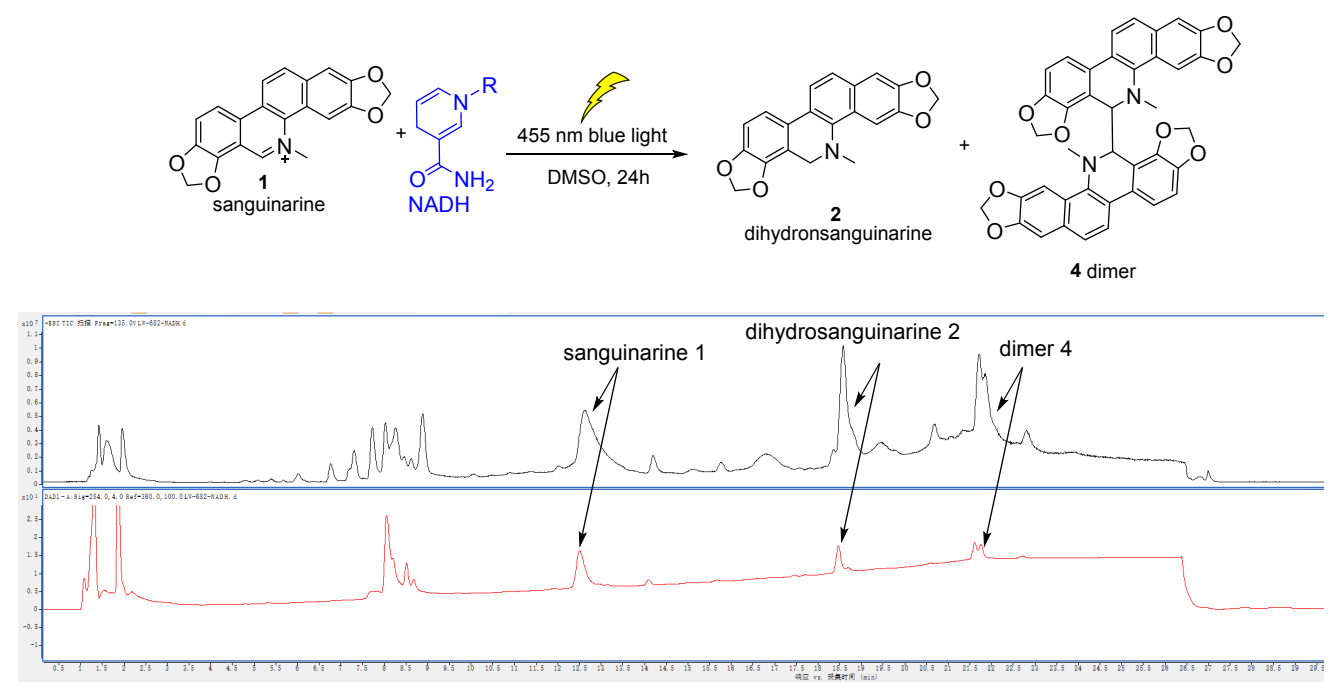

Figure S1. HPLC-HRMS results of light-mediated reduction of sanguinarine 1 by NADH

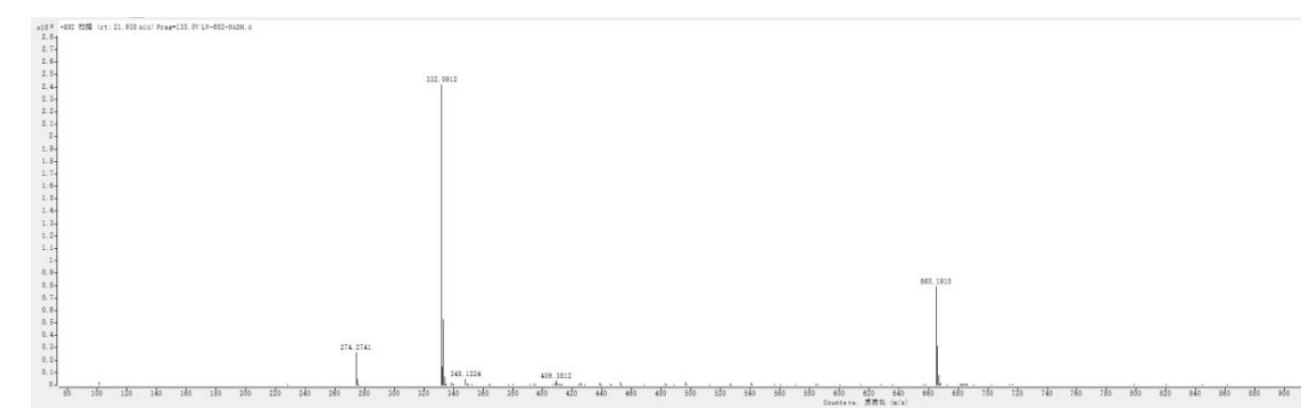

Figure S2. HRMS of dimer 4, calculated 665.1918 for $\mathrm{C}_{40} \mathrm{H}_{29} \mathrm{~N}_{2} \mathrm{O}_{8}{ }^{+}[\mathrm{M}+\mathrm{H}]^{+}$, found 665.1910

2. Photoreactor and general procedure for photoreaction

A Schlenk tube equipped with a magnetic stir bar (Figure S3) was charged with 0.2 mmol quaternary benzophenanthridine 1 or 8, 2.0 equivalent of a 6-substituted DHP 6 and $2 \mathrm{~mL}$ DMSO under $\mathrm{N}_{2}$ atmosphere. The flask was sealed by a plastic screw-cap with a Teflon sealed inlet for a glass rod. A high power LED $(\lambda=455 \mathrm{~nm})$ was attached to the top of the glass rod, which then could act as an optical fiber. After irradiation at room temperature for $24 \mathrm{~h}$, the LED was removed, the solvent was poured in $20 \mathrm{~mL} \mathrm{H}_{2} \mathrm{O}$ and extracted with $20 \mathrm{~mL}$ EtOAc for three times. The combined 
organic layer was then washed with $20 \mathrm{~mL} \mathrm{H}_{2} \mathrm{O}$ for three times and evaporated, to give a crude product, which was purified by silica gel chromatography and eluted with PE/EA to give target compounds 7.

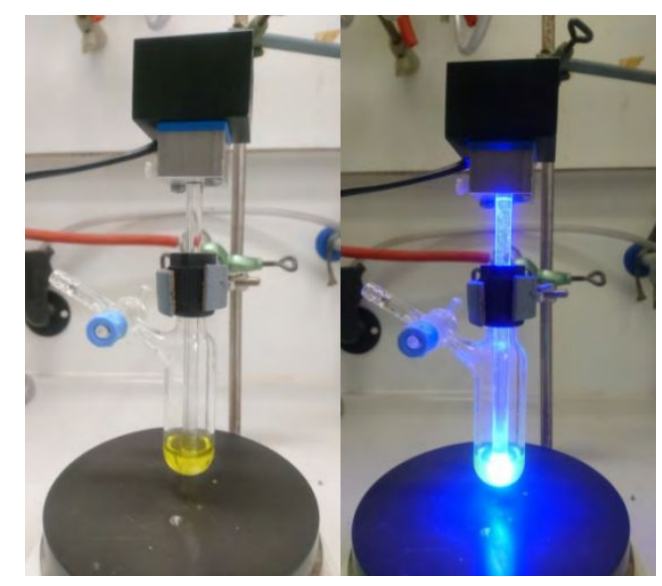

Figure S3. Photoreactor

3. Using primary 4-alkyl DHP $\mathbf{6 j}$ as reductant and alkylation reagent

We also evaluated the reaction efficacy with primary C-radicals as coupling partners. While the desired compounds were not detected, unexpectedly methylation to 19 apparently utilizing DMSO as the methyl source, had taken place instead. This hypothesis was confirmed with the generation of $6-\mathrm{CD}_{3}$ substituted dihydrosanguinarine under "standard" reaction conditions when deuterated DMSO (DMSO-d6) was used as solvent (Figure S4).
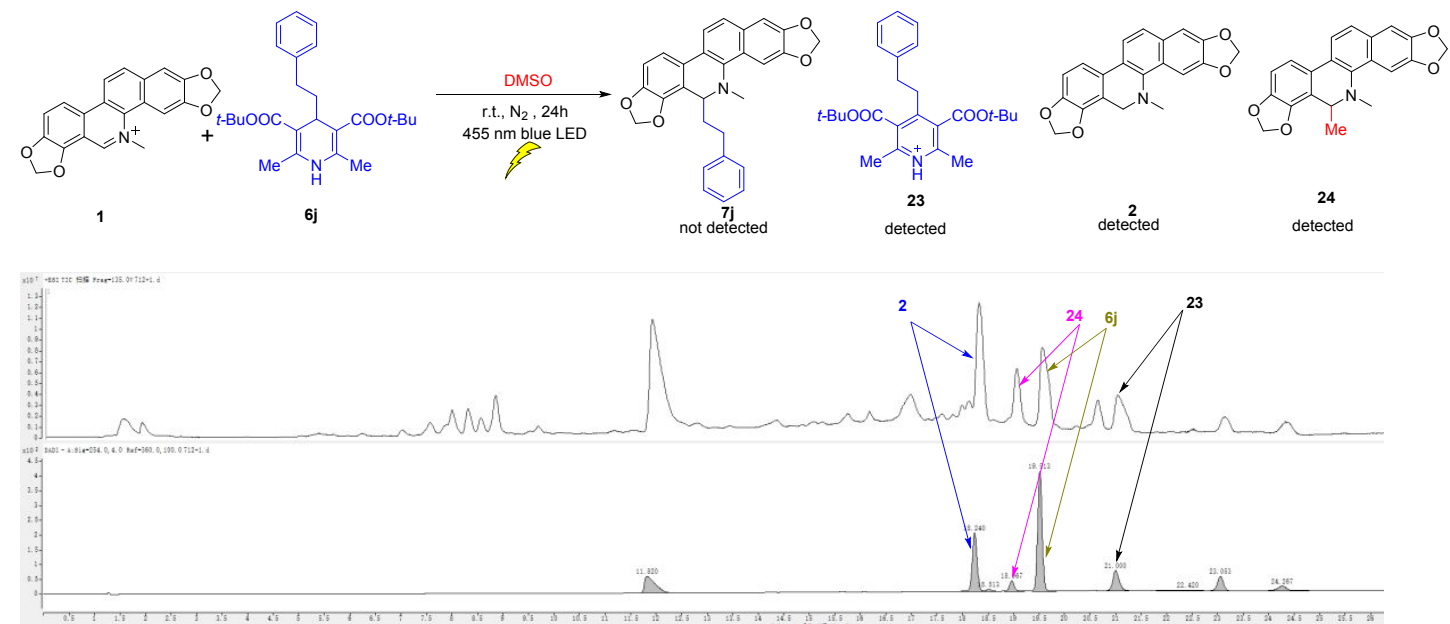


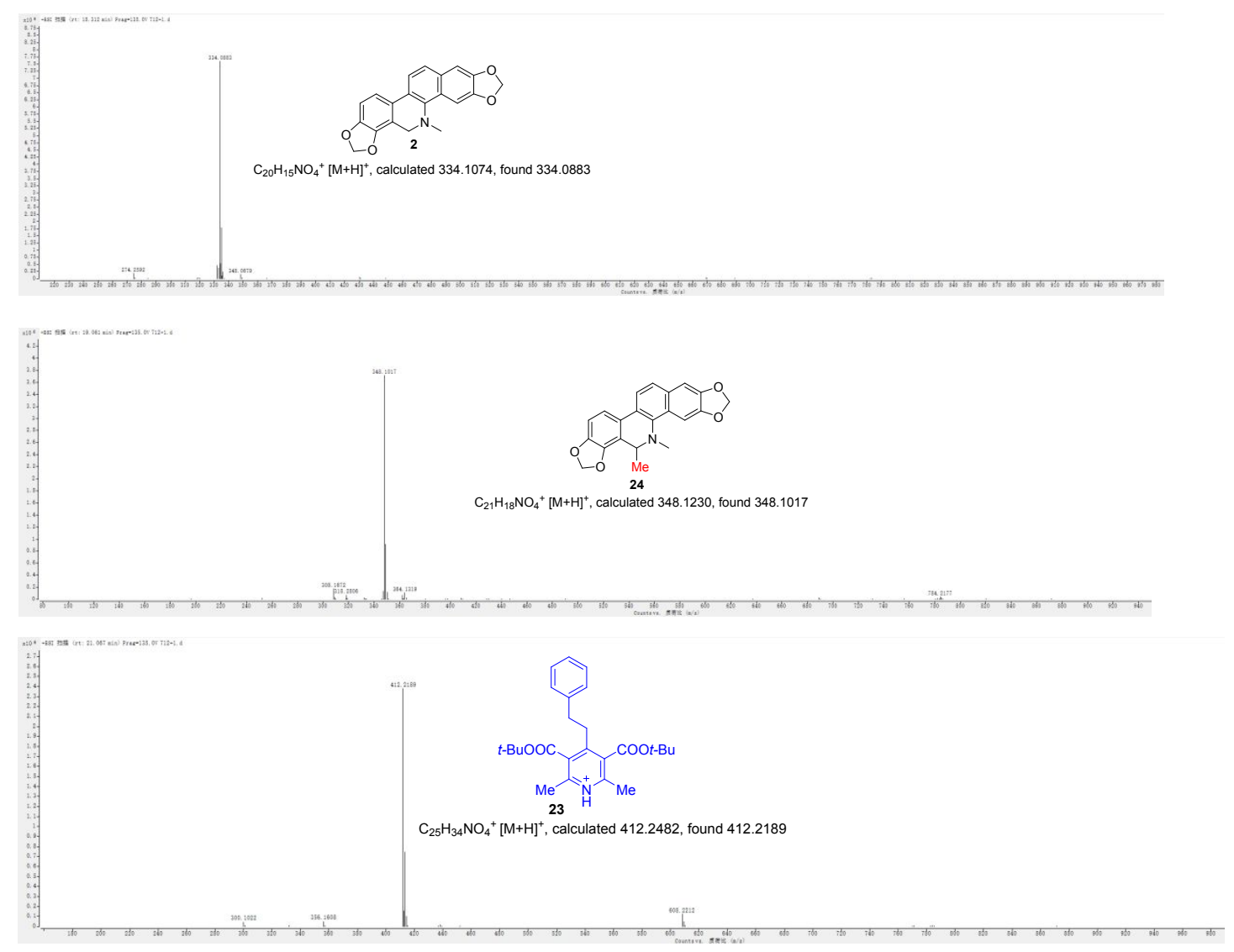

Figure S4. HPLL-MS results for 4-alkyl DHP 6j as reductant and alkylation reagent

4. NMR spectra data of target compounds

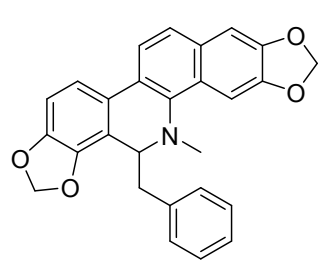

Compound 7a, obtained as pale yellow solid, ${ }^{1} \mathrm{H}$ NMR $(400 \mathrm{MHz}$, $\left.\mathrm{CDCl}_{3}\right): \delta 7.80(\mathrm{~d}, J=8.4 \mathrm{~Hz}, 1 \mathrm{H}), 7.59(\mathrm{~s}, 1 \mathrm{H}), 7.55(\mathrm{~d}, J=8.4$ $\mathrm{Hz}, 1 \mathrm{H}), 7.41(\mathrm{~d}, J=8.0 \mathrm{~Hz}, 1 \mathrm{H}), 7.28-7.24(\mathrm{~m}, 3 \mathrm{H}), 7.18(\mathrm{~s}, 1 \mathrm{H})$, $7.08(\mathrm{~d}, J=6.4 \mathrm{~Hz}, 2 \mathrm{H}), 6.90(\mathrm{~d}, J=8.4 \mathrm{~Hz}, 1 \mathrm{H}), 6.10(\mathrm{dd}, J=$ 15.2, 1.2 Hz, 2H), 5.98 (d, $J=1.6 \mathrm{~Hz}, 1 \mathrm{H}), 5.81(\mathrm{~d}, J=1.2 \mathrm{~Hz}, 1 \mathrm{H}), 4.46$ (dd, $J=8.8$, $6.0 \mathrm{~Hz}, 1 \mathrm{H}), 2.79-2.67(\mathrm{~m}, 2 \mathrm{H}), 2.61(\mathrm{~s}, 3 \mathrm{H}) .{ }^{13} \mathrm{C} \mathrm{NMR}\left(100 \mathrm{MHz}, \mathrm{CDCl}_{3}\right): \delta 148.0$, $147.5,146.9,144.7,140.0,139.3,131.1,129.8(\times 2), 127.8(2), 127.6,125.9,125.7$, $123.7,123.7,120.1,117.3,116.3,107.3,104.3,101.3(\times 2), 101.0,60.1,43.1,40.2$. HRMS (ESI ${ }^{+}$): calcd 424.1543 for $\mathrm{C}_{27} \mathrm{H}_{22} \mathrm{NO}_{4}{ }^{+}[\mathrm{M}+\mathrm{H}]^{+}$; found, 424.1543 .

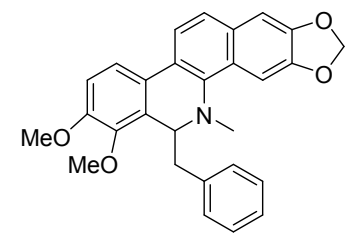

Compound 7aa, obtained as pale yellow solid, ${ }^{1} \mathrm{H}$ NMR (400MHz, $\left.\mathrm{CDCl}_{3}\right): \delta 7.81(\mathrm{~d}, J=8.4 \mathrm{~Hz}, 1 \mathrm{H}), 7.62(\mathrm{~d}, J=8.4$ $\mathrm{Hz}, 1 \mathrm{H})$, 7.56-7.53 (m, 2H), 7.33-7.27 (m, 3H), $7.18(\mathrm{~s}, 1 \mathrm{H})$, 
7.15-7.13 (m, 2H), $7.01(\mathrm{~d}, J=8.4 \mathrm{~Hz}, 1 \mathrm{H}), 6.12(\mathrm{~d}, J=1.2 \mathrm{~Hz}, 1 \mathrm{H}), 6.07$ (d, $J=1.2$ $\mathrm{Hz}, 1 \mathrm{H}), 4.68$ (dd, $J=10.8,6.8 \mathrm{~Hz}, 1 \mathrm{H}), 3.97$ (d, $J=4.8 \mathrm{~Hz}, 6 \mathrm{H}), 2.74-2.59$ (m, 2H), 2.56(s, 3H). ${ }^{13} \mathrm{C}$ NMR (100 MHz, $\left.\mathrm{CDCl}_{3}\right): \delta 152.2,147.9,147.5,145.9,140.2,140.1$, $131.2,129.8(\times 2), 129.8,127.8(\times 2), 127.4,125.8,125.0,123.6,123.5,119.8,118.7$, 111.5, 104.3, 101.4, 100.9, 60.8, 60.3, 55.9, 42.9, 40.3. HRMS (ESI $\left.{ }^{+}\right)$: calcd 440.1856 for $\mathrm{C}_{28} \mathrm{H}_{26} \mathrm{NO}_{4}{ }^{+}[\mathrm{M}+\mathrm{H}]^{+}$; found, 440.1857 .

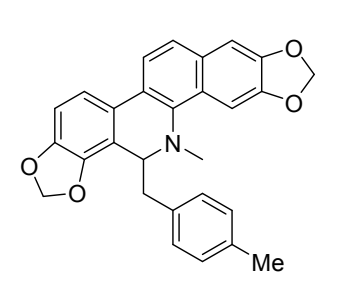

Compound 7b, obtained as white solid, ${ }^{1} \mathrm{H}$ NMR $(400 \mathrm{MHz}$, $\left.\mathrm{CDCl}_{3}\right): \delta 7.79(\mathrm{~d}, J=8.4 \mathrm{~Hz}, 1 \mathrm{H}), 7.61(\mathrm{~s}, 1 \mathrm{H}), 7.54(\mathrm{~d}, J=8.8$ $\mathrm{Hz}, 1 \mathrm{H}), 7.41$ (d, $J=8.0 \mathrm{~Hz}, 1 \mathrm{H}), 7.17(\mathrm{~s}, 1 \mathrm{H}), 7.10(\mathrm{~d}, J=8.0$ $\mathrm{Hz}, 2 \mathrm{H}), 6.98(\mathrm{~d}, J=8.0 \mathrm{~Hz}, 2 \mathrm{H}), 6.89(\mathrm{~d}, J=8.0 \mathrm{~Hz}, 1 \mathrm{H}), 6.12(\mathrm{~d}$, $J=1.2 \mathrm{~Hz}, 1 \mathrm{H}), 6.08(\mathrm{~d}, J=0.8 \mathrm{~Hz}, 1 \mathrm{H}), 5.99(\mathrm{~d}, J=1.2 \mathrm{~Hz}, 1 \mathrm{H}), 5.84(\mathrm{~d}, J=1.2 \mathrm{~Hz}$, $1 \mathrm{H}), 4.42(\mathrm{dd}, J=9.2,6.0 \mathrm{~Hz}, 1 \mathrm{H}), 2.73-2.65(\mathrm{~m}, 2 \mathrm{H}), 2.60(\mathrm{~s}, 3 \mathrm{H}), 2.38(\mathrm{~s}, 3 \mathrm{H}) .{ }^{13} \mathrm{C}$ NMR $\left(100 \mathrm{MHz}, \mathrm{CDCl}_{3}\right): \delta$ 148.0, 147.5, 146.9, 144.7, 140.1, 136.5, 135.2, 131.1(×2), $129.7(\times 2), 128.5(\times 2), 127.6,125.7,123.7,120.1,117.4,116.3,107.2$, 104.3, $101.3(\times 2), 101.0,60.2,43.2,39.8,21.0$. HRMS $\left(\mathrm{ESI}^{+}\right)$: calcd 438.1700 for $\mathrm{C}_{28} \mathrm{H}_{24} \mathrm{NO}_{4}^{+}[\mathrm{M}+\mathrm{H}]^{+}$; found, 438.1695 .

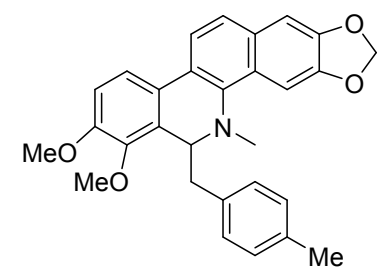

Compound $7 \mathbf{b b}$, obtained as white powder, ${ }^{1} \mathrm{H}$ NMR (400 $\left.\mathrm{MHz}, \mathrm{CDCl}_{3}\right): \delta 7.82(\mathrm{~d}, J=8.4 \mathrm{~Hz}, 1 \mathrm{H}), 7.62(\mathrm{~d}, J=8.4 \mathrm{~Hz}$, $1 \mathrm{H}), 7.60(\mathrm{~s}, 1 \mathrm{H}), 7.56(\mathrm{~d}, J=8.4 \mathrm{~Hz}, 1 \mathrm{H}), 7.20(\mathrm{~s}, 1 \mathrm{H})$, 7.15(d, $J=7.6 \mathrm{~Hz}, 2 \mathrm{H}), 7.07(\mathrm{~d}, J=8.0 \mathrm{~Hz}, 2 \mathrm{H}), 7.01(\mathrm{~d}, J=$ $8.8 \mathrm{~Hz}, 1 \mathrm{H}), 6.14(\mathrm{~d}, J=0.8 \mathrm{~Hz}, 1 \mathrm{H}), 6.09(\mathrm{~d}, J=0.8 \mathrm{~Hz}, 1 \mathrm{H}), 4.67$ (dd, $J=10.8,8$ $\mathrm{Hz}, 1 \mathrm{H}), 3.99$ (s, 6H), 2.71-2.66 (m, 2H), 2.58(s, 3H), 2.41 (s, 3H). ${ }^{13} \mathrm{C}$ NMR (100 $\left.\mathrm{MHz}, \mathrm{CDCl}_{3}\right): \delta 152.2,147.9,147.5,145.7,140.1,137.2,135.2,131.1,130.0,129.7$ $(\times 2), 128.6(\times 2), 127.4,124.8,123.7,123.6,119.9,118.8,111.2,104.3,101.4,101.0$, 60.9, 60.4, 55.8, 43.0, 39.8, 21.2. HRMS $\left(\mathrm{ESI}^{+}\right)$: calcd 454.2013 for $\mathrm{C}_{29} \mathrm{H}_{28} \mathrm{NO}_{4}{ }^{+}$ $[\mathrm{M}+\mathrm{H}]^{+}$; found, 454.2014 .

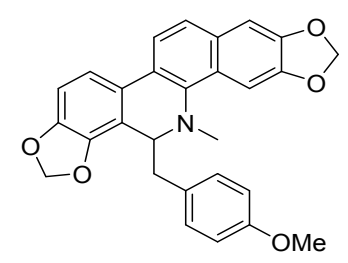

Compound 7c, obtained as white solid, ${ }^{1} \mathrm{H}$ NMR $(400 \mathrm{MHz}$, $\left.\mathrm{CDCl}_{3}\right): \delta 7.79(\mathrm{~d}, J=9.2 \mathrm{~Hz}, 1 \mathrm{H}), 7.61(\mathrm{~s}, 1 \mathrm{H}), 7.54(\mathrm{~d}, J=8.8$ $\mathrm{Hz}, 1 \mathrm{H}), 7.40$ (d, $J=8.0 \mathrm{~Hz}, 1 \mathrm{H}), 7.28(\mathrm{~s}, 1 \mathrm{H}), 7.17$ (s, 1H), 
$7.00(\mathrm{~d}, J=8.4 \mathrm{~Hz}, 2 \mathrm{H}), 7.00(\mathrm{~d}, J=8.4 \mathrm{~Hz}, 2 \mathrm{H}), 6.89$ (d, $J=8.0 \mathrm{~Hz}, 1 \mathrm{H}), 6.83$ (d, $J$ $=8.4 \mathrm{~Hz}, 2 \mathrm{H}), 6.12(\mathrm{~d}, J=1.2 \mathrm{~Hz}, 1 \mathrm{H}), 6.08(\mathrm{~d}, J=0.8 \mathrm{~Hz}, 1 \mathrm{H}), 5.99(\mathrm{~d}, J=1.2 \mathrm{~Hz}$, $1 \mathrm{H}), 5.85$ (d, $J=1.2 \mathrm{~Hz}, 1 \mathrm{H}), 4.40$ (dd, $J=8.8,6.0 \mathrm{~Hz}, 1 \mathrm{H}), 3.84(\mathrm{~s}, 3 \mathrm{H}), 2.72-2.63$ (m, 2H), $2.61(\mathrm{~s}, 3 \mathrm{H}) .{ }^{13} \mathrm{C}$ NMR (100 MHz, $\left.\mathrm{CDCl}_{3}\right): \delta 157.9,148.0,147.5,146.8$, $144.7,140.0,131.5,131.1,130.8(\times 2), 127.5,125.6,123.7(\times 2), 120.1,117.4,116.3$, 113.2(×2), 107.3, 104.3, 101.4, 101.3, 101.0, 60.3, 55.3, 43.2, 39.3. HRMS $\left(\mathrm{ESI}^{+}\right)$: calcd 454.1649 for $\mathrm{C}_{28} \mathrm{H}_{24} \mathrm{NO}_{5}{ }^{+}[\mathrm{M}+\mathrm{H}]^{+}$; found, 454.1649 .

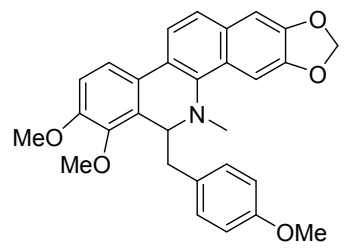

Compound 7cc, obtained as white solid, ${ }^{1} \mathrm{H}$ NMR $(400 \mathrm{MHz}$, $\left.\mathrm{CDCl}_{3}\right): \delta 7.80(\mathrm{~d}, J=8.8 \mathrm{~Hz}, 1 \mathrm{H}), 7.61(\mathrm{~d}, J=8.4 \mathrm{~Hz}, 1 \mathrm{H})$, $7.56(\mathrm{~s}, 1 \mathrm{H}), 7.53(\mathrm{~d}, J=8.8 \mathrm{~Hz}, 1 \mathrm{H}), 7.18(\mathrm{~s}, 1 \mathrm{H}), 7.06(\mathrm{~d}, J=$ $8.4 \mathrm{~Hz}, 2 \mathrm{H}), 7.00(\mathrm{~d}, J=8.4 \mathrm{~Hz}, 1 \mathrm{H}), 6.86(\mathrm{~d}, J=8.8 \mathrm{~Hz}, 2 \mathrm{H})$, $6.12(\mathrm{~d}, J=1.2 \mathrm{~Hz}, 1 \mathrm{H}), 6.07(\mathrm{~d}, J=1.2 \mathrm{~Hz}, 1 \mathrm{H}), 4.62(\mathrm{dd}, J=10.4,4 \mathrm{~Hz}, 1 \mathrm{H})$, 3.97(s, 6H), 3.85(s, 3H), 2.68-2.53 (m, 2H), 2.57 (s, 3H). ${ }^{13} \mathrm{C}$ NMR (100 MHz, $\left.\mathrm{CDCl}_{3}\right): \delta 157.9,152.1,147.9,147.5,145.7,140.1,132.4,131.1,130.7(\times 2), 130.0$, $127.3,124.8,123.6,123.5,119.9,118.8,113.3(\times 2), 111.2,104.3,101.4,101.0,60.9$, 60.4, 55.8, 55.3, 43.0, 39.3. HRMS $\left(\mathrm{ESI}^{+}\right)$: calcd 470.1962 for $\mathrm{C}_{29} \mathrm{H}_{28} \mathrm{NO}_{5}{ }^{+}[\mathrm{M}+\mathrm{H}]^{+}$; found, 470.1963 .

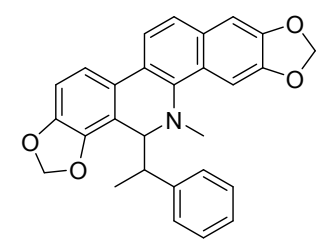

Compound 7d , obtained as white solid, diastereomeric ratio is $4: 3,{ }^{1} \mathrm{H}$ NMR $\left(400 \mathrm{MHz}, \mathrm{CDCl}_{3}\right): \delta 7.84(\mathrm{~s}, 1 \mathrm{H}), 7.81(\mathrm{~d}, J=8.4$ $\mathrm{Hz}, 1 \mathrm{H}), 7.76(\mathrm{~d}, J=8.4 \mathrm{~Hz}, 1 \mathrm{H}), 7.55(\mathrm{~d}, J=8.4 \mathrm{~Hz}, 1 \mathrm{H}), 7.50$ $(\mathrm{d}, J=8.4 \mathrm{~Hz}, 1 \mathrm{H}), 7.41(\mathrm{~d}, J=8.4 \mathrm{~Hz}, 1 \mathrm{H}), 7.36(\mathrm{~d}, J=8.4 \mathrm{~Hz}$, 1H), 7.27-7.25 (m, 3H), 7.19-7.13 (m, 4H), 7.03-6.94 (m, 6H), 6.78(d, $J=8.0 \mathrm{~Hz}$, $1 \mathrm{H}), 6.14-6.09(\mathrm{~m}, 4 \mathrm{H}), 6.01(\mathrm{~d}, J=1.2 \mathrm{~Hz}, 1 \mathrm{H}), 5.74(\mathrm{~d}, J=1.2 \mathrm{~Hz}, 1 \mathrm{H}), 5.07(\mathrm{~d}, J=$ $1.2 \mathrm{~Hz}, 1 \mathrm{H}), 4.36$ (d, $J=10.4 \mathrm{~Hz}, 1 \mathrm{H}), 4.14(\mathrm{~d}, J=10 \mathrm{~Hz}, 1 \mathrm{H}), 2.74(\mathrm{~s}, 3 \mathrm{H}), 2.6-2.60$ $(\mathrm{m}, 2 \mathrm{H}), 2.48(\mathrm{~s}, 3 \mathrm{H}), 1.47(\mathrm{~d}, J=6.8 \mathrm{~Hz}, 3 \mathrm{H}), 1.22(\mathrm{~d}, J=7.6 \mathrm{~Hz}, 3 \mathrm{H}) .{ }^{13} \mathrm{C} \mathrm{NMR}$ $\left(100 \mathrm{MHz}, \mathrm{CDCl}_{3}\right): \delta 148.2,147.6,147.5,147.4,146.7,146.3,145.9,1145.8,144.1$, $140.2,139.8,131.0(\times 2), 128.6,127.8(\times 2), 127.4(\times 2), 127.2,126.0(\times 2), 125.9(\times 2)$, $125.9,125.5,124.2,123.9,123.7,123.4,120.1,119.9,116.8,116.3,116.2,107.5$, 107.0, 104.5, 104.0, 101.7, 101.2, 101.1, 101.0, 100.9, 64.7, 64.1, 43.2, 43.0, 42.9, 
42.8, 18.6, 18.0. HRMS (ESI ${ }^{+}$): calcd 438.1700 for $\mathrm{C}_{28} \mathrm{H}_{24} \mathrm{NO}_{4}{ }^{+}[\mathrm{M}+\mathrm{H}]^{+}$; found, 438.1704 .

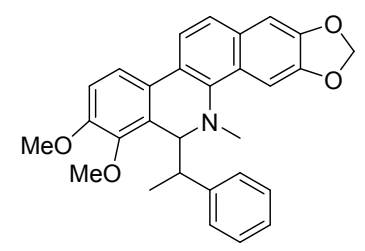

Compound 7dd, obtained as pale yellow solid, obtained as white solid, diastereomeric ratio is 5:4, ${ }^{1} \mathrm{H}$ NMR (400 MHz, $\left.\mathrm{CDCl}_{3}\right): \delta 7.83-7.76(\mathrm{~m}, 2 \mathrm{H}), 7.60-7.48(\mathrm{~m}, 2 \mathrm{H}), 7.22-7.13(\mathrm{~m}$, 4H), 7.06-6.91 (m, 3H), 6.10-6.01 (m, 2H), 4.58-4.48 (m, 1H), 4.04-3.42 (6H) including $4.04(\mathrm{~s}), 4.00(\mathrm{~s}), 3.83(\mathrm{~s})$ and 3.41(s), 2.78-2.48 (m. 1H), 2.74-2.48 (3H) including 2.74(s) and 2.48(s), 1.28-1.15 (3H) including $1.27(\mathrm{~d}, J=$ $7.8 \mathrm{~Hz})$ and $1.16(\mathrm{~d}, J=7.6 \mathrm{~Hz}) \cdot{ }^{13} \mathrm{C} \mathrm{NMR}\left(100 \mathrm{MHz}, \mathrm{CDCl}_{3}\right): \delta 151.9,151.8,148.0$, $147.5,147.4(\times 2), 147.0,146.5,146.3,144.9,140.6,140.3,131.0(\times 2), 128.5(\times 2)$, $127.8,127.7(\times 6), 127.1,127.0,125.9,125.7,125.2,125.1,124.4,123.9,123.3,123.2$, $119.8,119.7,119.1,118.3,111.6,111.2,104.5,140.1,101.6,101.0(\times 2), 100.9$, 63.8(×2), 60.9, 60.1, 55.9, 55.8, 43.8, 42.9, 42.6, 42.4, 18.7, 17.6. HRMS $\left(\mathrm{ESI}^{+}\right)$: calcd 454.2013 for $\mathrm{C}_{29} \mathrm{H}_{28} \mathrm{NO}_{4}^{+}[\mathrm{M}+\mathrm{H}]^{+}$; found, 454.2016.

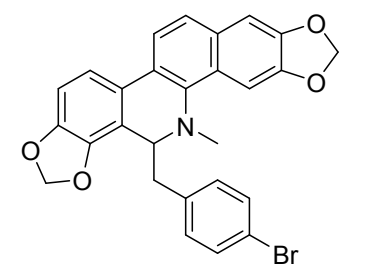

Compound 7e, obtained as yellow white solid, ${ }^{1} \mathrm{H}$ NMR (400 $\left.\mathrm{MHz}, \mathrm{CDCl}_{3}\right): \delta 7.76(\mathrm{~d}, J=8.8 \mathrm{~Hz}, 1 \mathrm{H}), 7.55-7.52(\mathrm{~m}, 2 \mathrm{H})$, 7.40-7.37 (m, 3H), $7.16(\mathrm{~s}, 1 \mathrm{H}), 6.94-6.88(\mathrm{~m}, 3 \mathrm{H}), 6.12$ (d, $J=$ $0.8 \mathrm{~Hz}, 1 \mathrm{H}), 6.08(\mathrm{~d}, J=1.2 \mathrm{~Hz}, 1 \mathrm{H}), 5.99(\mathrm{~d}, J=1.2 \mathrm{~Hz}, 1 \mathrm{H})$, $5.85(\mathrm{~d}, J=1.2 \mathrm{~Hz}, 1 \mathrm{H}), 4.39(\mathrm{dd}, J=9.2,6.0 \mathrm{~Hz}, 1 \mathrm{H}), 2.71-2.63(\mathrm{~m}, 2 \mathrm{H}), 2.60$ (s, 3H). $\left.{ }^{13} \mathrm{C} \mathrm{NMR} \mathrm{(100} \mathrm{MHz,} \mathrm{CDCl}_{3}\right): \delta 148.1,147.6,146.9,144.7,139.7,138.3,131.6$ $(\times 2), 131.1,130.8(\times 2), 127.4,125.6,123.9,123.6,120.1,119.8,116.8,116.4,107.4$, 104.4, 101.4, 101.1 $(\times 2), 59.9,43.2$, 39.5. HRMS $\left(\mathrm{ESI}^{+}\right)$: calcd 502.0648 for $\mathrm{C}_{27} \mathrm{H}_{21} \mathrm{BrNO}_{4}^{+}[\mathrm{M}+\mathrm{H}]^{+}$; found, 502.0643.

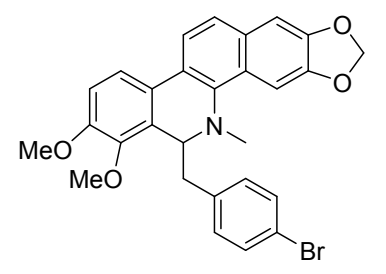

Compound 7ee, obtained as white solid, ${ }^{1} \mathrm{H}$ NMR (400 MHz, $\left.\mathrm{CDCl}_{3}\right): \delta 7.78(\mathrm{~d}, J=8.8 \mathrm{~Hz}, 1 \mathrm{H}), 7.60(\mathrm{~d}, J=8.4 \mathrm{~Hz}, 1 \mathrm{H})$, $7.53(\mathrm{~d}, J=8.8 \mathrm{~Hz}, 1 \mathrm{H}), 7.50$ (s, 1H), 7.41 (d, $J=8.4 \mathrm{~Hz}, 2 \mathrm{H})$, $7.17(\mathrm{~s}, 1 \mathrm{H}), 7.00(\mathrm{dd}, J=8.0,2.4 \mathrm{~Hz}, 3 \mathrm{H}), 6.13(\mathrm{~s}, 1 \mathrm{H}), 6.08$ (s, 1H), $4.61(\mathrm{dd}, J=10.4,3.6 \mathrm{~Hz}, 1 \mathrm{H}), 3.97$ (s, 3H), 3.97 (s, 3H), 2.67-2.51 (m, 2H), 
$2.55(\mathrm{~s}, 3 \mathrm{H}) .{ }^{13} \mathrm{C} \mathrm{NMR}\left(100 \mathrm{MHz}, \mathrm{CDCl}_{3}\right): \delta 152.1,148.0,147.5,145.8,139.8,139.1$, $131.5(\times 2), 131.2,130.8(\times 2), 129.4,127.3,124.9,123.6(\times 2), 119.8,119.7,118.7$, 111.6, 104.4, 101.1, 101.0, 60.8, 60.1, 55.9, 42.9, 39.6. HRMS (ESI $\left.{ }^{+}\right)$: calcd 518.0961 for $\mathrm{C}_{28} \mathrm{H}_{25} \mathrm{BrNO}_{4}^{+}[\mathrm{M}+\mathrm{H}]^{+}$; found, 518.0964.

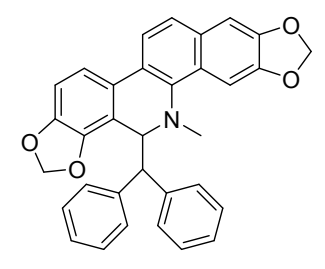

Compound 7f, obtained as pale yellow solid, ${ }^{1} \mathrm{H}$ NMR $(400 \mathrm{MHz}$, $\left.\mathrm{CDCl}_{3}\right): \delta 7.82(\mathrm{~d}, J=8.8 \mathrm{~Hz}, 1 \mathrm{H}), 7.51(\mathrm{~d}, J=8.4 \mathrm{~Hz}, 1 \mathrm{H}), 7.40$ $(\mathrm{d}, J=8.0 \mathrm{~Hz}, 2 \mathrm{H}), 7.29$ (s, 3H), 7.25-7.23 (m, 3H), 7.11-7.08 (m, 3H), 7.01-6.99 (m, 2H), $6.79(\mathrm{~d}, J=6.0 \mathrm{~Hz}, 1 \mathrm{H}), 5.98(\mathrm{dd}, J=$ 14.0, 1.2 Hz, 2H), 5.78 (d, $J=1.6 \mathrm{~Hz}, 1 \mathrm{H}), 5.10$ (d, $J=1.6 \mathrm{~Hz}, 1 \mathrm{H}), 4.93$ (d, $J=11.2$ $\mathrm{Hz}, 1 \mathrm{H}), 3.81(\mathrm{~d}, J=11.2 \mathrm{~Hz}, 1 \mathrm{H}), 2.64(\mathrm{~s}, 3 \mathrm{H}) .{ }^{13} \mathrm{C} \mathrm{NMR}\left(100 \mathrm{MHz}, \mathrm{CDCl}_{3}\right): \delta$ $147.7,147.4,146.4,145.5,142.4,141.8,139.5,131.0,129.4(\times 2), 128.7(\times 2), 127.9$ $(\times 2), 127.6,127.4(\times 2), 125.9(\times 2), 125.8,123.9,123.5,119.8,116.3,115.5,107.2$, 103.9, 101.2, 100.9, 100.7, 61.4, 54.8, 42.4. HRMS $\left(\mathrm{ESI}^{+}\right)$: calcd 500.1856 for $\mathrm{C}_{33} \mathrm{H}_{26} \mathrm{NO}_{4}^{+}[\mathrm{M}+\mathrm{H}]^{+}$; found, 500.1858 .

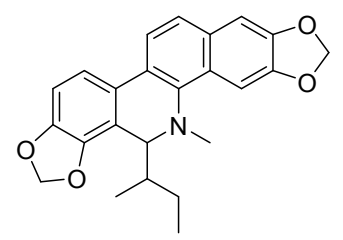

Compound 7e, obtained as white solid, diastereomeric ratio is $1: 1,{ }^{1} \mathrm{H}$ NMR (400 MHz, $\left.\mathrm{CDCl}_{3}\right): \delta 7.76-7.71(\mathrm{~m}, 2 \mathrm{H}), 7.48(\mathrm{~d}, J$ $=8.4 \mathrm{~Hz}, 1 \mathrm{H}), 7.34(\mathrm{~d}, J=8.0 \mathrm{~Hz}, 1 \mathrm{H}), 7.13(\mathrm{~s}, 1 \mathrm{H}), 6.88(\mathrm{dd}, J$ $=8.4,1.2 \mathrm{~Hz}, 1 \mathrm{H}), 6.09-6.01(\mathrm{~m}, 4 \mathrm{H}), 3.93-3.87(2 \mathrm{H})$, including $3.92(\mathrm{~d}, J=9.6 \mathrm{~Hz})$ and $3.98(\mathrm{~d}, J=9.2 \mathrm{~Hz}), 2.67-2.77(3 \mathrm{H})$ including $2.67(\mathrm{~s})$ and $2.66(\mathrm{~s}), 1.45-1.15(\mathrm{~m}, 3 \mathrm{H}), 0.97-0.77(\mathrm{~m}, 6 \mathrm{H}) .{ }^{13} \mathrm{C} \mathrm{NMR}\left(100 \mathrm{MHz}, \mathrm{CDCl}_{3}\right): \delta 148.0$ $(\times 2), 147.4,146.7,145.6,145.5,140.9,140.6,131.0,127.3(\times 2), 126.2,126.1,124.4$, $124.3,123.4,120.1,120.0,117.2,116.6,107.0,104.4,101.0(\times 2), 100.9(\times 2), 63.1$, 62.4, 43.1, 42.9, 38.4, 37.0, 25.8, 25.6, 16.0, 15.5, 11.5, 10.2. HRMS (ESI $\left.{ }^{+}\right)$: calcd 390.1700 for $\mathrm{C}_{24} \mathrm{H}_{24} \mathrm{NO}_{4}{ }^{+}[\mathrm{M}+\mathrm{H}]^{+}$; found, 390.1704 .

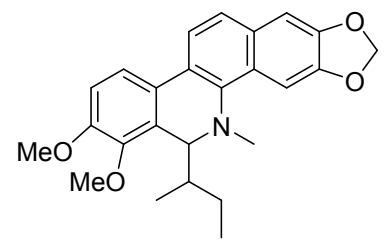

Compound 7ee, obtained as white solid, diastereomeric ratio is $1: 1,{ }^{1} \mathrm{H}$ NMR $\left(400 \mathrm{MHz}, \mathrm{CDCl}_{3}\right): \delta 7.77-7.73(\mathrm{~m}, 2 \mathrm{H})$, 7.54(dd, $J=8.4,2.0 \mathrm{~Hz}, 1 \mathrm{H}), 7.48(\mathrm{~d}, J=8.4 \mathrm{~Hz}, 1 \mathrm{H}), 7.14$ (s, 1H), $6.97(\mathrm{dd}, J=8.4,1.2 \mathrm{~Hz}, 1 \mathrm{H}), 6.08(\mathrm{~s}, 2 \mathrm{H}), 4.16-4.10$ 
$(1 \mathrm{H})$ including $4.14(\mathrm{~d}, \mathrm{~J}=9.2 \mathrm{~Hz})$ and $4.11(\mathrm{~d}, \mathrm{~J}=8.4 \mathrm{~Hz}), 3.96(\mathrm{~s}, 3 \mathrm{H}), 3.94(\mathrm{~s}, 3 \mathrm{H})$, 2.68-2.67 (3H) including $2.68(\mathrm{~s})$ and $2.67(\mathrm{~s}), 1.38-1.20(\mathrm{~m}, 3 \mathrm{H}), 0.93-0.74(\mathrm{~m}, 6 \mathrm{H})$. ${ }^{13} \mathrm{C}$ NMR $\left(100 \mathrm{MHz}, \mathrm{CDCl}_{3}\right): \delta 151.9,147.9(\times 2), 147.3(\times 2), 146.8(\times 2), 140.8,140.7$, $130.9,128.9,128.8,127.2,127.1,125.1,125.0,124.2,124.3,123.2,119.8(\times 2)$, $119.0(\times 2), 110.8,104.5,104.4,101.0,100.9(\times 2), 62.7,62.0,60.8,55.7,42.8,42.5$, 38.5, 36.9, 25.9, 25.7, 16.2, 15.7, 11.9, 10.5. HRMS $\left(\mathrm{ESI}^{+}\right)$: calcd 406.2013 for $\mathrm{C}_{25} \mathrm{H}_{28} \mathrm{NO}_{4}^{+}[\mathrm{M}+\mathrm{H}]^{+}$; found, 406.2017.

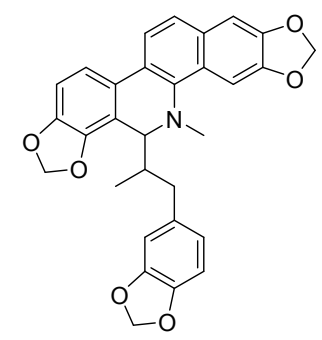

Compound 7f, obtained as pale yellow solid, diastereomeric ratio is $1: 1,{ }^{1} \mathrm{H}$ NMR $\left(400 \mathrm{MHz}, \mathrm{CDCl}_{3}\right): \delta 7.80-7.72(\mathrm{~m}, 2 \mathrm{H})$, $7.50(\mathrm{~m}, 1 \mathrm{H}), 7.36(\mathrm{~m}, 1 \mathrm{H}), 7.17-7.13(1 \mathrm{H})$ including $7.17(\mathrm{~s})$ and 7.13 (s), 6.90-6.87 (m, 1H), 6.66-6.34 (m, 1H), 6.49-6.40 (m, 2H), 6.09-6.00 (m, 4H), 5.88-5.86 (m, 2H), 3.98-3.89 (1H) including $3.97(\mathrm{~d}, J=8.8 \mathrm{~Hz})$ and $3.91(\mathrm{~d}, J=10.4 \mathrm{~Hz}), 2.84-2.61(\mathrm{~m}, 1 \mathrm{H}), 2.69(3 \mathrm{H})$ including $2.69(\mathrm{~s})$ and $2.68(\mathrm{~s}), 2.46-2.30(\mathrm{~m}, 1 \mathrm{H}), 1.74-1.56(\mathrm{~m}, 1 \mathrm{H}), 0.82-0.72(3 \mathrm{H})$ including $0.81(\mathrm{~d}, J=6.4 \mathrm{~Hz})$ and $0.73(\mathrm{~d}, J=6.8 \mathrm{~Hz}) \cdot{ }^{13} \mathrm{C} \mathrm{NMR}\left(100 \mathrm{MHz}, \mathrm{CDCl}_{3}\right): \delta 148.2$, $148.1,147.5,147.4,147.3,147.2,146.7(\times 2), 145.6,145.4,145.3,140.8,140.2$, 135.2, $135.0,131.1,127.3,127.2,126.2,126.0,124.5,124.3,123.7,123.5,122.3,121.8$, $120.1(\times 2), 116.8,116.7(\times 2), 109.8,109.4,107.8(\times 2), 107.3,107.2,104.5,104.4$, $101.1,101.0(\times 2), 100.9,100.8,100.6(\times 2), 100.5,62.8,62.6,43.4,42.6,39.6,39.5$, 39.3, 38.1, 16.2, 15.8. HRMS (ESI ${ }^{+}$): calcd 496.1755 for $\mathrm{C}_{30} \mathrm{H}_{26} \mathrm{NO}_{6}{ }^{+}[\mathrm{M}+\mathrm{H}]^{+}$; found, 496.1757.

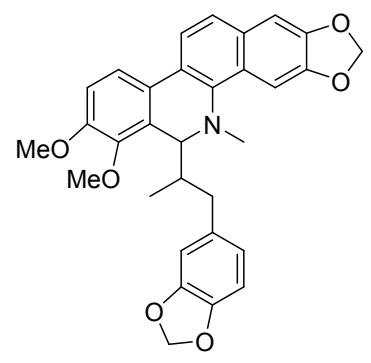

Compound 7ff, obtained as white powder, diastereomeric ratio is 5:4, ${ }^{1} \mathrm{H}$ NMR $\left(400 \mathrm{MHz}, \mathrm{CDCl}_{3}\right): \delta 7.81-7.75(\mathrm{~m}, 2 \mathrm{H})$, 7.59-7.47(m, 2H), 7.18-7.16 (1H) including $7.18(\mathrm{~s})$ and 7.14 (s), 6.9-6.96 (m, 1H), 6.69-6.55 (m, 2H), 6.40-6.31 (m, 1H), 6.09-6.08 (m, 2H), 5.89-5.84 (m, 2H), 4.19-4.15 (m, 1H), 3.98-3.89 (m, 6H), 2.77-2.57(m, 4H), 2.43-2.30 (m, 1H), 1.80-1.50 (m, 1H), 0.71-0.68 $(\mathrm{m}, 3 \mathrm{H}) .{ }^{13} \mathrm{C} \mathrm{NMR}\left(100 \mathrm{MHz}, \mathrm{CDCl}_{3}\right): \delta 152.0,151.9,148.0(\times 2), 147.4(\times 2), 147.2$, $147.1,146.8,145.2(\times 2), 141.2,140.3,135.7,135.6,131.0,128.6,128.3,127.2,126.9$, 
$125.4,125.0,124.4,124.2,123.6,123.3, \quad 122.2,121.9,119.9,119.8,119.1,118.8$, $111.0(\times 2), 109.7,109.5,107.7(\times 2), 104.5(\times 2), 101.0(\times 2), 100.8,100.6(\times 2), 100.5$, $62.4,61.7,60.8,60.7,55.7,43.4,42.3,39.9,39.7,39.6,38.3,16.1,15.9$. HRMS $\left(\mathrm{ESI}^{+}\right)$: calcd 512.2068 for $\mathrm{C}_{31} \mathrm{H}_{30} \mathrm{NO}_{6}{ }^{+}[\mathrm{M}+\mathrm{H}]^{+}$; found, 512.2070 .

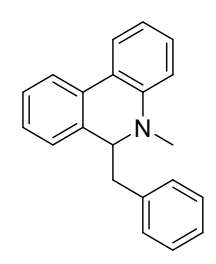

Compound 12, obtained as white powder, ${ }^{1} \mathrm{H}$ NMR (400 MHz, $\left.\mathrm{CDCl}_{3}\right): \delta$ $7.80(\mathrm{~m}, 2 \mathrm{H}), 7.34-7.29(\mathrm{~m}, 2 \mathrm{H}), 7.25-7.20$ (m, 3H), 7.08 (t, $J=7.6 \mathrm{~Hz}$, $1 \mathrm{H}), 7.00-6.90(\mathrm{~m}, 3 \mathrm{H}), 6.73(\mathrm{~d}, J=8.0 \mathrm{~Hz}, 1 \mathrm{H}), 6.60(\mathrm{~d}, J=7.2 \mathrm{~Hz}, 1 \mathrm{H})$, $4.43(\mathrm{dd}, J=8.0,2.0 \mathrm{~Hz}, 1 \mathrm{H}), 3.02-2.97(\mathrm{~m}, 1 \mathrm{H}), 2.99$ (s, 3H), 2.70-2.64 (m, $1 \mathrm{H})$. calcd 286.1590 for $\mathrm{C}_{21} \mathrm{H}_{20} \mathrm{~N}^{+}[\mathrm{M}+\mathrm{H}]^{+}$; found, 286.1583 .

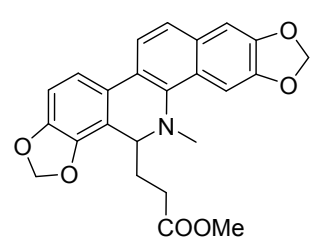

Compound 16a: obtained as white solid, ${ }^{1} \mathrm{H}$ NMR $(500 \mathrm{MHz}$, $\left.\mathrm{CDCl}_{3}\right): \delta 7.71(\mathrm{~d}, J=8.5 \mathrm{~Hz}, 1 \mathrm{H}), 7.71(\mathrm{~s}, 1 \mathrm{H}), 7.50(\mathrm{~d}, J=8.5$ $\mathrm{Hz}, 1 \mathrm{H}), 7.35$ (d, $J=8.0 \mathrm{~Hz}, 1 \mathrm{H}), 7.14(\mathrm{~s}, 1 \mathrm{H}), 6.88$ (d, $J=8.0 \mathrm{~Hz}$, $1 \mathrm{H}), 6.08(\mathrm{~s}, 3 \mathrm{H}), 6.05(\mathrm{~d}, J=1.5 \mathrm{~Hz}, 1 \mathrm{H}), 4.32(\mathrm{dd}, J=5.5,10.0$ $\mathrm{Hz}, 1 \mathrm{H}), 3.65$ (s, 3H), 2.67 (s, 3H), 2.59-2.46 (m, 2H), 1.83-1.68 (m, 2H). ${ }^{13} \mathrm{CNMR}$ (125 MHz, $\left.\mathrm{CDCl}_{3}\right): \delta 174.1,148.2,147.5,147.0,144.7,139.6,131.0,127.5,125.6$, 123.9, 123.6, 120.1, 117.3, 116.5, 107.4, 104.4, 101.4, 101.0, 100.7, 56.8, 51.5, 43.1, 30.4, 28.6. HRMS (ESI ${ }^{+}$): calcd 420.1442 for $\mathrm{C}_{24} \mathrm{H}_{22} \mathrm{NO}_{6}{ }^{+}[\mathrm{M}+\mathrm{H}]^{+}$; found, 420.1438 .

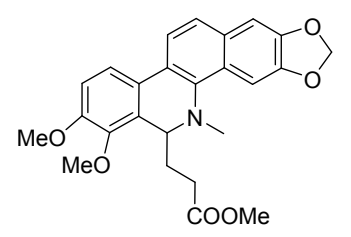

Compound 16aa: obtained as white solid, ${ }^{1} \mathrm{H}$ NMR $(400 \mathrm{MHz}$, $\left.\mathrm{CDCl}_{3}\right): \delta 7.73(\mathrm{~s}, 1 \mathrm{H}), 7.71(\mathrm{~s}, 1 \mathrm{H}), 7.55(\mathrm{~d}, J=8.8 \mathrm{~Hz}, 1 \mathrm{H})$, $7.49(\mathrm{~d}, J=8.8 \mathrm{~Hz}, 1 \mathrm{H}), 7.13(\mathrm{~s}, 1 \mathrm{H}), 6.96(\mathrm{~d}, J=8.8 \mathrm{~Hz}, 1 \mathrm{H})$, 6.07 (s, 2H), $4.50(\mathrm{dd}, J=10.4,4.8 \mathrm{~Hz}, 1 \mathrm{H}), 3.97$ (s, 3H), 3.95 $(\mathrm{s}, 3 \mathrm{H}), 3.64(\mathrm{~s}, 3 \mathrm{H}), 2.66(\mathrm{~s}, 3 \mathrm{H}), 2.62-2.47(\mathrm{~m}, 2 \mathrm{H}), 1.80-1.61(\mathrm{~m}, 2 \mathrm{H}) .{ }^{13} \mathrm{C} \mathrm{NMR}$ $\left(100 \mathrm{MHz}, \mathrm{CDCl}_{3}\right): \delta 174.2,152.2,148.1,147.5,146.0,139.6,131.0,129.7,127.4$, 124.8, 123.7, 123.6, 119.9, 118.8, 111.4, 104.4, 101.0, 100.7, 61.0, 56.9, 55.8, 51.3, 42.7, 30.8, 29.1. HRMS (ESI ${ }^{+}$): calcd 436.1755 for $\mathrm{C}_{25} \mathrm{H}_{26} \mathrm{NO}_{6}{ }^{+}[\mathrm{M}+\mathrm{H}]^{+}$; found, $436.1764,436.1759$.

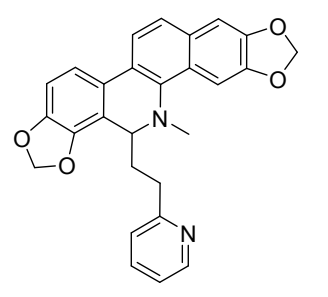

Compound 16b: obtained as white solid, ${ }^{1} \mathrm{H}$ NMR $(400 \mathrm{MHz}$, $11 / 42$ 
$\left.\mathrm{CDCl}_{3}\right): \delta 8.48(\mathrm{~d}, J=4.4 \mathrm{~Hz}, 1 \mathrm{H}), 7.78(\mathrm{~s}, 1 \mathrm{H}), 7.71(\mathrm{~d}, J=8.4 \mathrm{~Hz}, 1 \mathrm{H}), 7.53-7.47$ (m, 2H), $7.35(\mathrm{~d}, J=8.0 \mathrm{~Hz}, 1 \mathrm{H}), 7.13(\mathrm{~s}, 1 \mathrm{H}), 7.09-7.03(\mathrm{~m}, 2 \mathrm{H}), 6.86(\mathrm{~d}, J=8.0$ $\mathrm{Hz}, 1 \mathrm{H}), 6.06-6.01(\mathrm{~m}, 4 \mathrm{H}), 4.30-4.27(\mathrm{~m}, 1 \mathrm{H}), 3.10-2.92(\mathrm{~m}, 2 \mathrm{H}), 2.68(\mathrm{~s}, 3 \mathrm{H})$, 1.94-1.85 (m, 2H). ${ }^{13} \mathrm{C}$ NMR (100 MHz, $\left.\mathrm{CDCl}_{3}\right): \delta 162.0,149.1,148.1,147.5,147.0$, 144.6, 139.9, 136.1, 131.0, 127.6, 125.8, 123.8, 123.7, 122.8, 120.8, 120.2, 117.8, 116.4, 107.2, 104.4, 101.3, 101.0, 100.8, 57.4, 43.0, 34.9, 33.6. HRMS (ESI $\left.{ }^{+}\right)$: calcd 439.1652 for $\mathrm{C}_{27} \mathrm{H}_{23} \mathrm{~N}_{2} \mathrm{O}_{4}^{+}[\mathrm{M}+\mathrm{H}]^{+}$; found, 439.1654 .

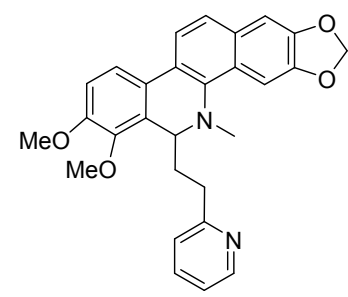

Compound 16bb: obtained as white solid, ${ }^{1} \mathrm{H}$ NMR (400 MHz, $\left.\mathrm{CDCl}_{3}\right): \delta 8.50(\mathrm{~d}, J=4.4 \mathrm{~Hz}, 1 \mathrm{H}), 7.79(\mathrm{~s}, 1 \mathrm{H}), 7.73(\mathrm{~d}, J=8.4$ $\mathrm{Hz}, 1 \mathrm{H}), 7.56-7.48$ (m, 3H), 7.13-7.12 (d, m, 2H), 7.06 (dd, $J=$ 6.8, $5.2 \mathrm{~Hz}, 1 \mathrm{H}), 6.94(\mathrm{~d}, J=8.8 \mathrm{~Hz}, 1 \mathrm{H}), 6.07$ (s, 2H), 4.44 (dd, $J=10.0,4.8 \mathrm{~Hz}, 1 \mathrm{H}), 3.93(\mathrm{~s}, 3 \mathrm{H}), 3.87(\mathrm{~s}, 3 \mathrm{H}), 3.04(\mathrm{t}, J=7.6 \mathrm{~Hz}, 2 \mathrm{H}), 2.66(\mathrm{~s}$, $3 \mathrm{H}), 1.88-1.79(\mathrm{~m}, 2 \mathrm{H}) .{ }^{13} \mathrm{C} \mathrm{NMR}\left(100 \mathrm{MHz}, \mathrm{CDCl}_{3}\right): \delta 162.4,152.2,149.0,148.0$, $147.4,145.9,140.0,136.0,131.0,130.3,127.5,124.9,123.8,123.5,122.9,120.7$, 119.9, 118.8, 111.2, 104.4, 101.0, 100.8, 60.8, 57.1, 55.8, 42.7, 34.9, 33.7. HRMS (ESI ${ }^{+}$): calcd 455.1965 for $\mathrm{C}_{28} \mathrm{H}_{27} \mathrm{~N}_{2} \mathrm{O}_{4}^{+}[\mathrm{M}+\mathrm{H}]^{+}$; found, 455.1961 .

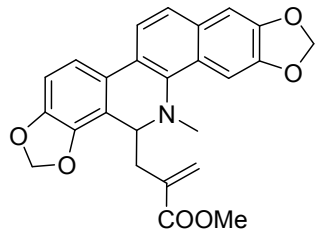

Compound 16c: Obtained as white solid, ${ }^{1} \mathrm{H}$ NMR $(500 \mathrm{MHz}$, $\left.\mathrm{CDCl}_{3}\right): \delta 7.71(\mathrm{~d}, J=8.5 \mathrm{~Hz}, 1), 7.6(\mathrm{~s}, 1 \mathrm{H}), 7.47(\mathrm{~d}, J=8.5 \mathrm{~Hz}$, $1 \mathrm{H}), 7.34(\mathrm{~d}, J=8.5 \mathrm{~Hz}, 1), 7.10(\mathrm{~s}, 1 \mathrm{H}), 6.85(\mathrm{~d}, \mathrm{~J}=8.5 \mathrm{~Hz}$, $1 \mathrm{H}), 6.14(\mathrm{~d}, \mathrm{~J}=1.0 \mathrm{~Hz}, 1 \mathrm{H}), 6.06-6.01(\mathrm{~m}, 4 \mathrm{H}), 5.15(\mathrm{~s}, 1 \mathrm{H})$, $4.57(\mathrm{dd}, \mathrm{J}=6.5,9.0 \mathrm{~Hz}, 1 \mathrm{H}), 3.76(\mathrm{~s}, 3 \mathrm{H}), 2.62$ (s, 3H), 2.44-2.34 (m, 2H). ${ }^{13} \mathrm{C} \mathrm{NMR}$ $\left(125 \mathrm{MHz}, \mathrm{CDCl}_{3}\right): \delta 167.6,147.9,147.4,146.8,144.8,139.6,136.7,130.9,128.0$, $127.4,125.4,123.7,123.4,120.0,116.9,116.2$, 107.2, 104.2, 101.2, 101.0, 56.6, 51.8, 43.0, 36.5. HRMS (ESI ${ }^{+}$): calcd 432.1442 for $\mathrm{C}_{25} \mathrm{H}_{22} \mathrm{NO}_{6}{ }^{+}[\mathrm{M}+\mathrm{H}]^{+}$; found, 432.1448 .

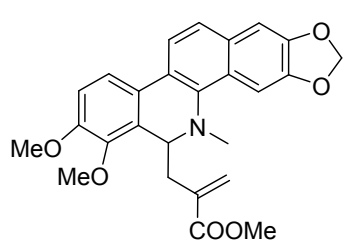

Compound 16ce: Obtained as white solid, ${ }^{1} \mathrm{H}$ NMR (400 MHz, $\left.\mathrm{CDCl}_{3}\right): \delta 7.75(\mathrm{~d}, J=8.4 \mathrm{~Hz}, 1 \mathrm{H}), 7.60(\mathrm{~s}, 1 \mathrm{H}), 7.56(\mathrm{~d}, J=$ $8.8 \mathrm{~Hz}, 1 \mathrm{H}), 7.49$ (d, $J=8.8 \mathrm{~Hz}, 1 \mathrm{H}), 7.13(\mathrm{~s}, 1 \mathrm{H}), 6.96$ (d, $J=$ $8.8 \mathrm{~Hz}, 1 \mathrm{H}), 6.17(\mathrm{~d}, J=1.6 \mathrm{~Hz}, 1 \mathrm{H}), 6.06(\mathrm{dd}, J=9.2,1.2 \mathrm{~Hz}$, 
2H), $5.15(\mathrm{~d}, J=1.2 \mathrm{~Hz}, 1 \mathrm{H}), 4.77$ (dd, $J=10,4.8 \mathrm{~Hz}, 1 \mathrm{H}), 3.99$ (s, 3H), 3.94(s, 3H), 3.77 (s, 3H), 2.62 (s, 3H), 2.48-2.27 (m, 2H). $\left.{ }^{13} \mathrm{C} \mathrm{NMR} \mathrm{(100} \mathrm{MHz,} \mathrm{CDCl}_{3}\right): \delta 167.7$, $152.2,147.8,147.4,146.1,139.8,137.3,131.0,129.4,127.5,127.3,124.8,123.5$, $123.5,119.8,118.7,111.4,104.3,101.1,101.0,60.9$, 56.9, 55.8, 51.6, 42.7, 36.6. HRMS $\left(\mathrm{ESI}^{+}\right)$: calcd 448.1755 for $\mathrm{C}_{26} \mathrm{H}_{26} \mathrm{NO}_{6}{ }^{+}[\mathrm{M}+\mathrm{H}]^{+}$; found, 448.1755.

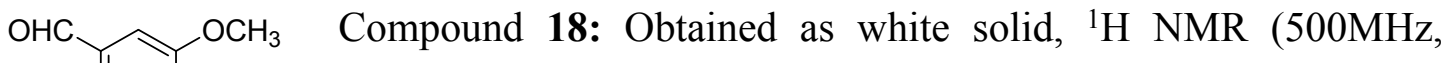
OAc $\quad \mathrm{CDCl} 3): \delta 9.87(\mathrm{~s}, 1 \mathrm{H}), 7.45(\mathrm{~s}, 1 \mathrm{H}), 7.42(\mathrm{~d}, \mathrm{~J}=7.5 \mathrm{~Hz}, 1 \mathrm{H}), 7.16$ $(\mathrm{d}, \mathrm{J}=7.5 \mathrm{~Hz}, 1 \mathrm{H}), 3.85$ (s, 3H), 2.29 (s, 3H). ${ }^{13} \mathrm{C} \mathrm{NMR}(125 \mathrm{MHz}, \mathrm{CDCl} 3): \delta 190.9$, $168.2,151.8,144.8,135.0,124.4,123.2,110.7,55.9,20.4$.

$\mathrm{OCH}_{3}$ Compound 19: Obtained as white solid, ${ }^{1} \mathrm{H}$ NMR $(500 \mathrm{MHz}$, OAc $\left.\mathrm{CDCl}_{3}\right): \delta 7.05(\mathrm{~s}, 1 \mathrm{H}), 7.02(\mathrm{~s}, 2 \mathrm{H}), 6.70(\mathrm{dd}, \mathrm{J}=17.0,11.0 \mathrm{~Hz}$, 1H), $5.74(\mathrm{~d}, \mathrm{~J}=17.5 \mathrm{~Hz}, 1 \mathrm{H}), 5.28(\mathrm{~d}, \mathrm{~J}=10.5 \mathrm{~Hz}, 1 \mathrm{H}), 3.86(\mathrm{~s}, 3 \mathrm{H}), 2.33$ (s, 3H). ${ }^{13} \mathrm{C}$ NMR $\left(125 \mathrm{MHz}, \mathrm{CDCl}_{3}\right): \delta 168.7,150.9,139.2,136.4,136.0,122.5,118.6,113.8$, $109.7,55.5,20.3$.

$\mathrm{OCH}_{3}$ Compound 20: Obtained as white solid, ${ }^{1} \mathrm{H}$ NMR $\left(500 \mathrm{MHz}, \mathrm{CDCl}_{3}\right): \delta 7.96(\mathrm{~d}, \mathrm{~J}=6.0 \mathrm{~Hz}, 2 \mathrm{H}), 7.67-7.58(\mathrm{~m}$, 4H), $7.10(\mathrm{~s}, 1 \mathrm{H}), 7.07$ (s, 2H), 6.85 (d, J = $15.0 \mathrm{~Hz}, 1 \mathrm{H}) .3 .86(\mathrm{~s}, 3 \mathrm{H}), 2.33$ (s, 3H). ${ }^{13} \mathrm{C} \mathrm{NMR}\left(125 \mathrm{MHz}, \mathrm{CDCl}_{3}\right): \delta 168.5,151.5,142.1,141.8,140.6,133.4,131.2,129.3$ $\times 2,127.6 \times 2,127.4,123.4,121.7,111.7,55.9,20.5$.

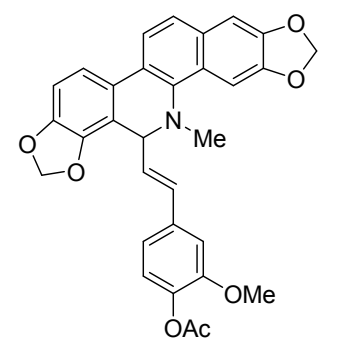

Compound 21. Obtained as white amorphous powder, ${ }^{1} \mathrm{H}$ NMR $\left(500 \mathrm{MHz}, \mathrm{CDCl}_{3}\right): \delta 7.80(\mathrm{~s}, 1 \mathrm{H}), 7.72(\mathrm{~d}, \mathrm{~J}=8.5 \mathrm{~Hz}, 1 \mathrm{H}), 7.49$ $(\mathrm{d}, \mathrm{J}=8.5 \mathrm{~Hz}, 1 \mathrm{H}), 7.40(\mathrm{~d}, \mathrm{~J}=8.0 \mathrm{~Hz}, 1 \mathrm{H}), 7.12(\mathrm{~s}, 1 \mathrm{H}), 6.94(\mathrm{~d}$, $\mathrm{J}=8.0 \mathrm{~Hz}, 1 \mathrm{H}), 6.83(\mathrm{~d}, \mathrm{~J}=8.0 \mathrm{~Hz}, 1 \mathrm{H}), 6.76(\mathrm{~s}, 1 \mathrm{H}), 6.73(\mathrm{~d}, \mathrm{~J}$ $=7.5 \mathrm{~Hz}, 1 \mathrm{H}), 6.32(\mathrm{~d}, \mathrm{~J}=15.5 \mathrm{~Hz}, 1 \mathrm{H}), 6.12-6.08(\mathrm{~m}, 5 \mathrm{H}), 5.04$ (br.s, 1H), $3.75(\mathrm{~s}, 3 \mathrm{H}), 2.77(\mathrm{~s}, 3 \mathrm{H}), 2.27(\mathrm{~s}, 3 \mathrm{H}) .{ }^{13} \mathrm{C} \mathrm{NMR}\left(125 \mathrm{MHz}, \mathrm{CDCl}_{3}\right): \delta$ $168.9,150.8,148.1,147.5,147.1,145.0,140.4,138.9,136.0,130.9,129.6,129.2$, 
$127.3,126.0,123.9,123.8,122.4,120.1,119.2,116.5,115.5,110.2,107.6,104.4$, 101.5, 101.0, 100.8, 59.1, 55.8, 42.8, 20.6. HRMS $\left(\mathrm{ESI}^{+}\right)$: calcd 524.1704 for $\mathrm{C}_{31} \mathrm{H}_{26} \mathrm{NO}_{7}^{+}[\mathrm{M}+\mathrm{H}]^{+}$; found, 524.1708. 
5. NMR spectra of target compounds

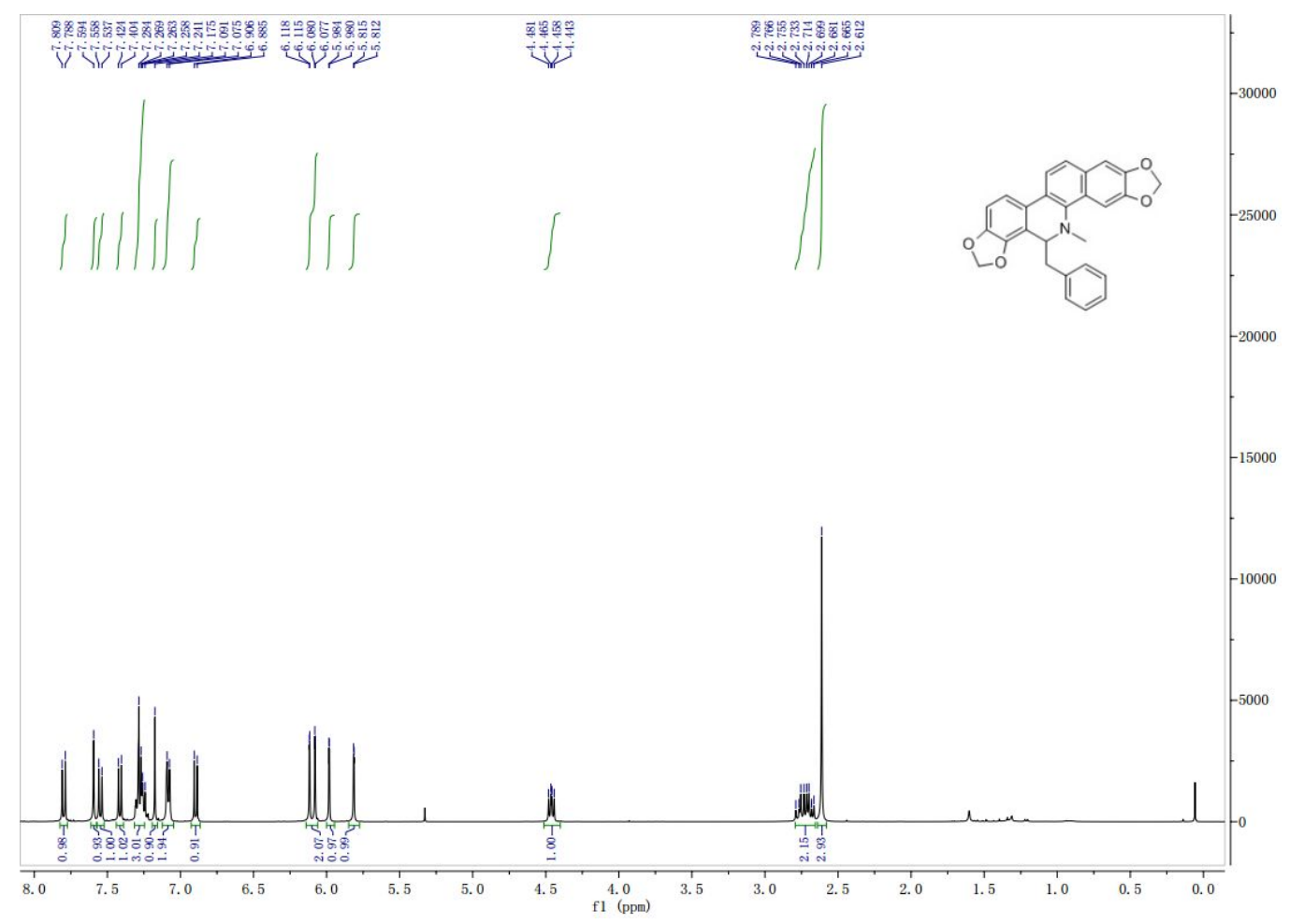

Figure S5. ${ }^{1} \mathrm{H}$ NMR spectrum $\left(400 \mathrm{MHz}, \mathrm{CDCl}_{3}\right)$ of $7 \mathbf{a}$

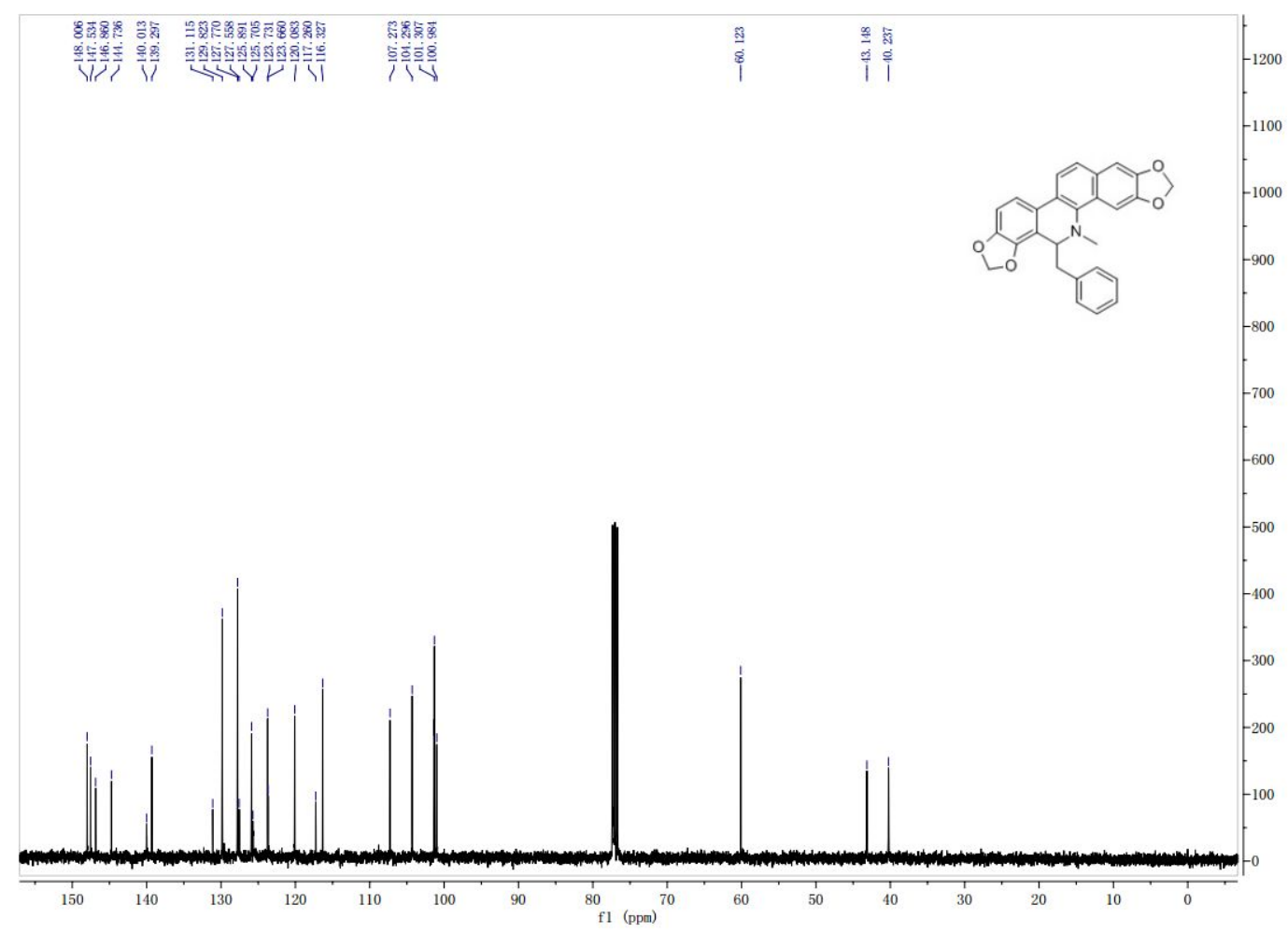

Figure S6. ${ }^{13} \mathrm{C} \mathrm{NMR}$ spectrum $\left(400 \mathrm{MHz}, \mathrm{CDCl}_{3}\right)$ of $7 \mathbf{a}$ 


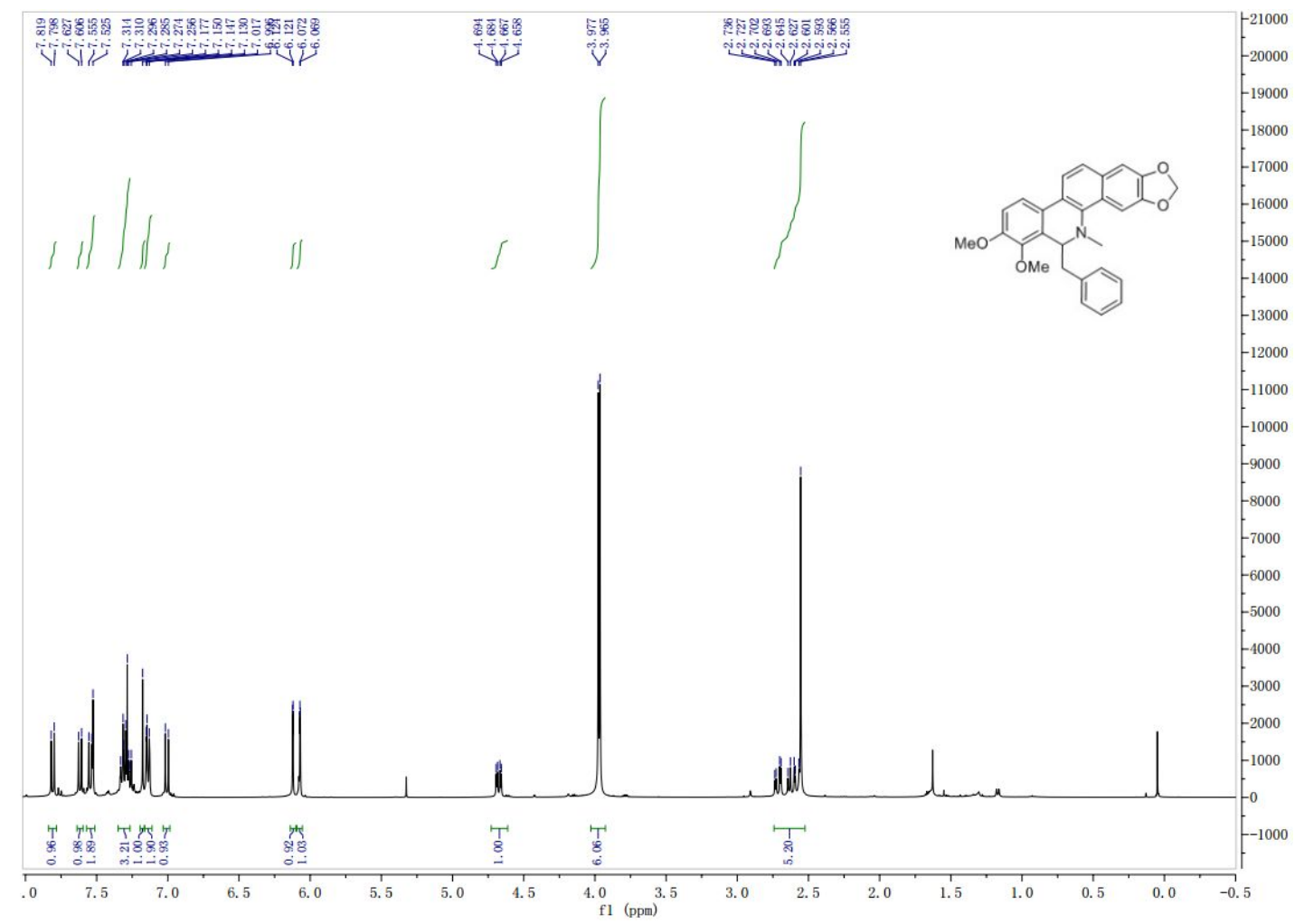

Figure S7. ${ }^{1} \mathrm{H}$ NMR spectrum $\left(400 \mathrm{MHz}, \mathrm{CDCl}_{3}\right)$ of $7 \mathbf{a a}$

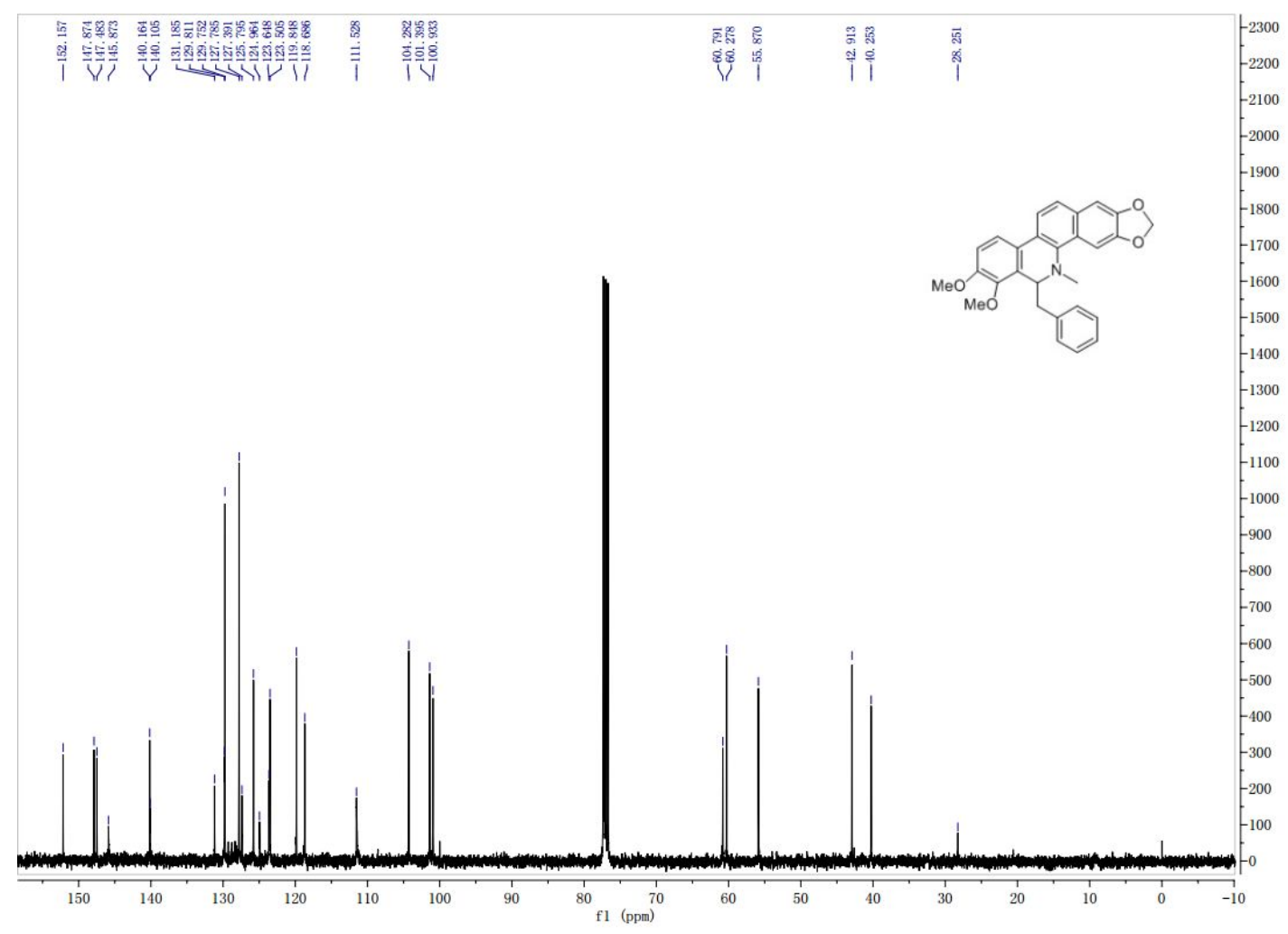

Figure S8. ${ }^{13} \mathrm{C}$ NMR spectrum $\left(400 \mathrm{MHz}, \mathrm{CDCl}_{3}\right)$ of 7aa 


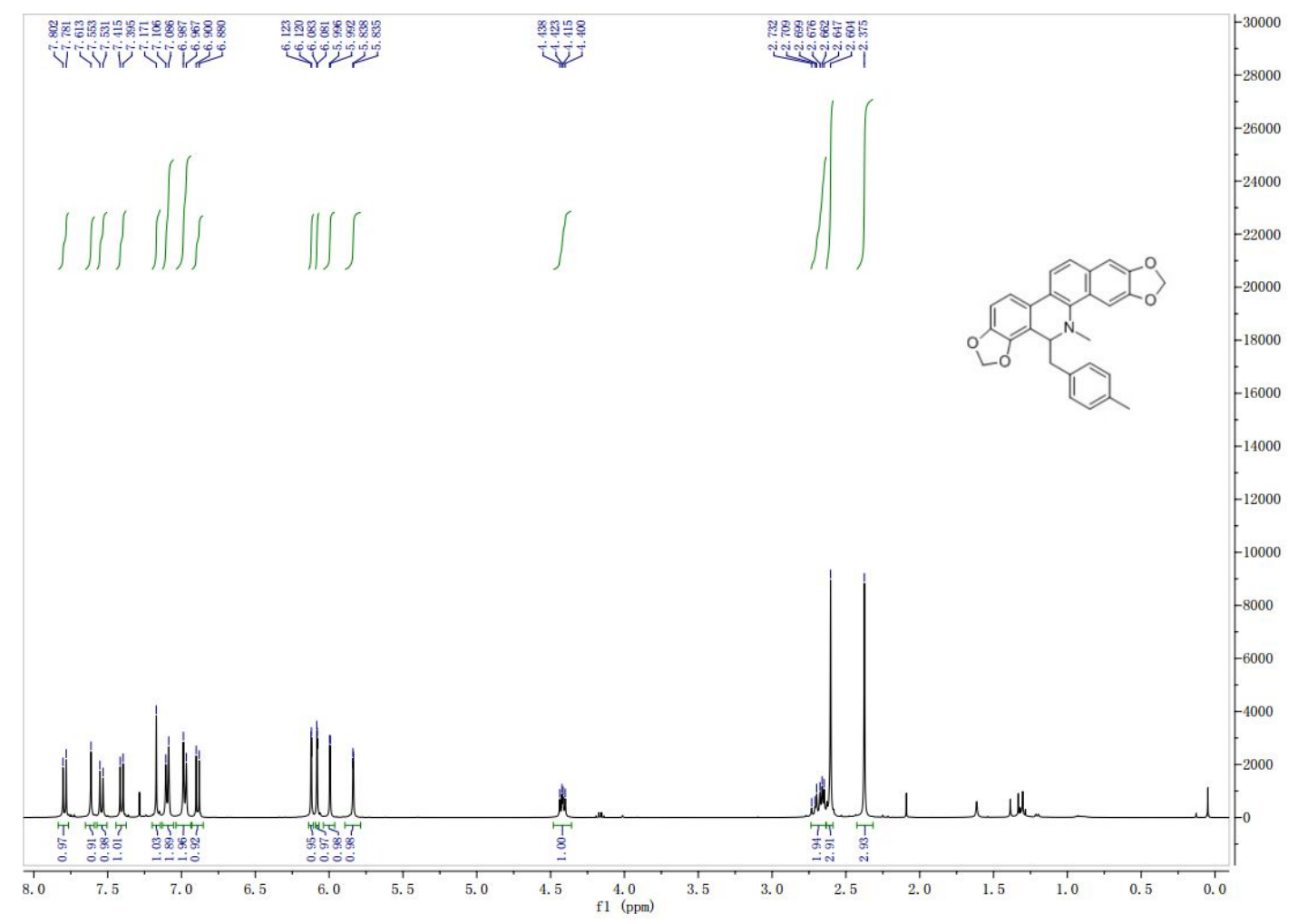

Figure S9. ${ }^{1} \mathrm{H} \mathrm{NMR}$ spectrum $\left(400 \mathrm{MHz}, \mathrm{CDCl}_{3}\right)$ of $\mathbf{7 b}$

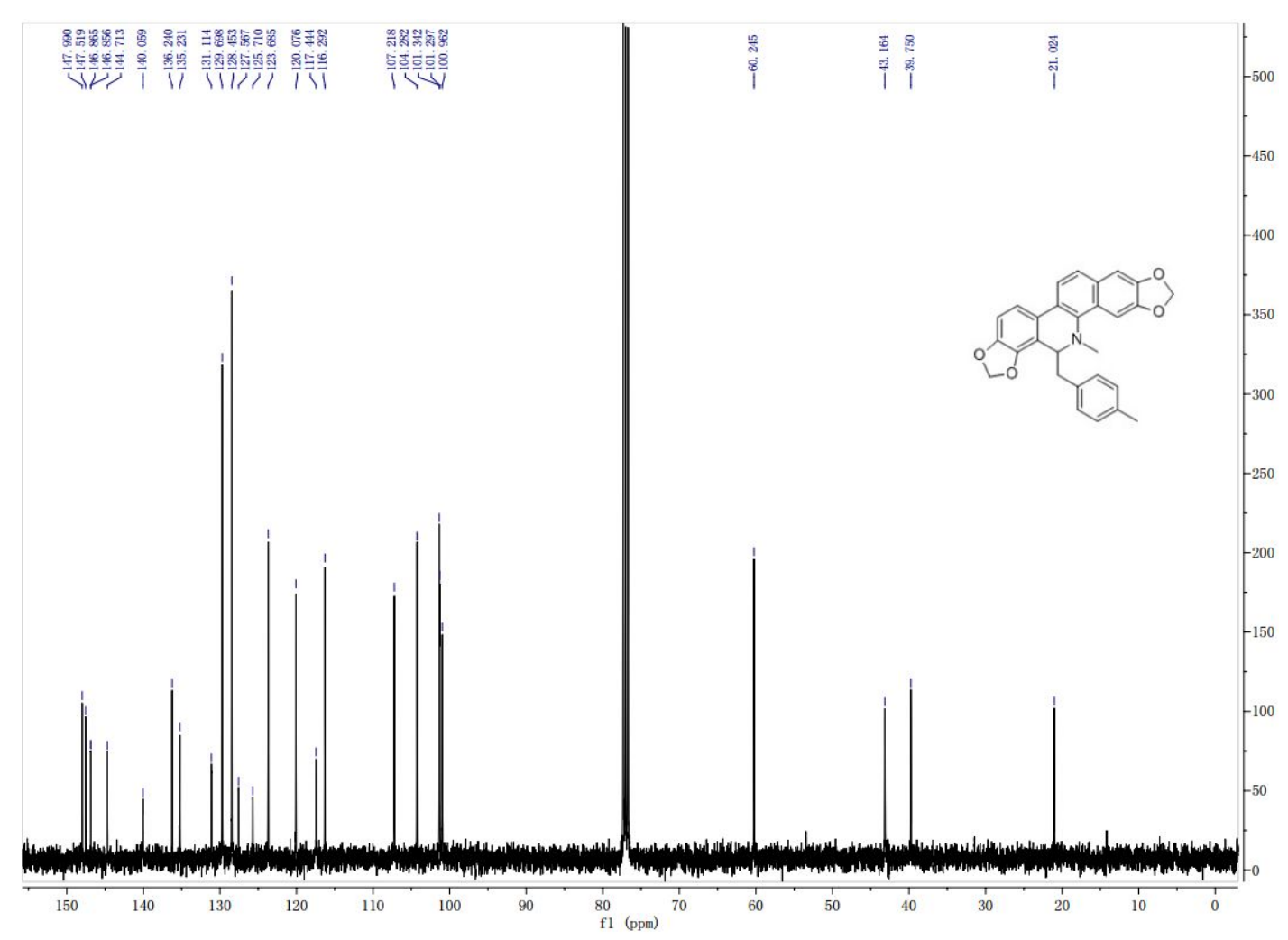

Figure S10. ${ }^{13} \mathrm{C}$ NMR spectrum $\left(400 \mathrm{MHz}, \mathrm{CDCl}_{3}\right)$ of $7 \mathbf{b}$ 


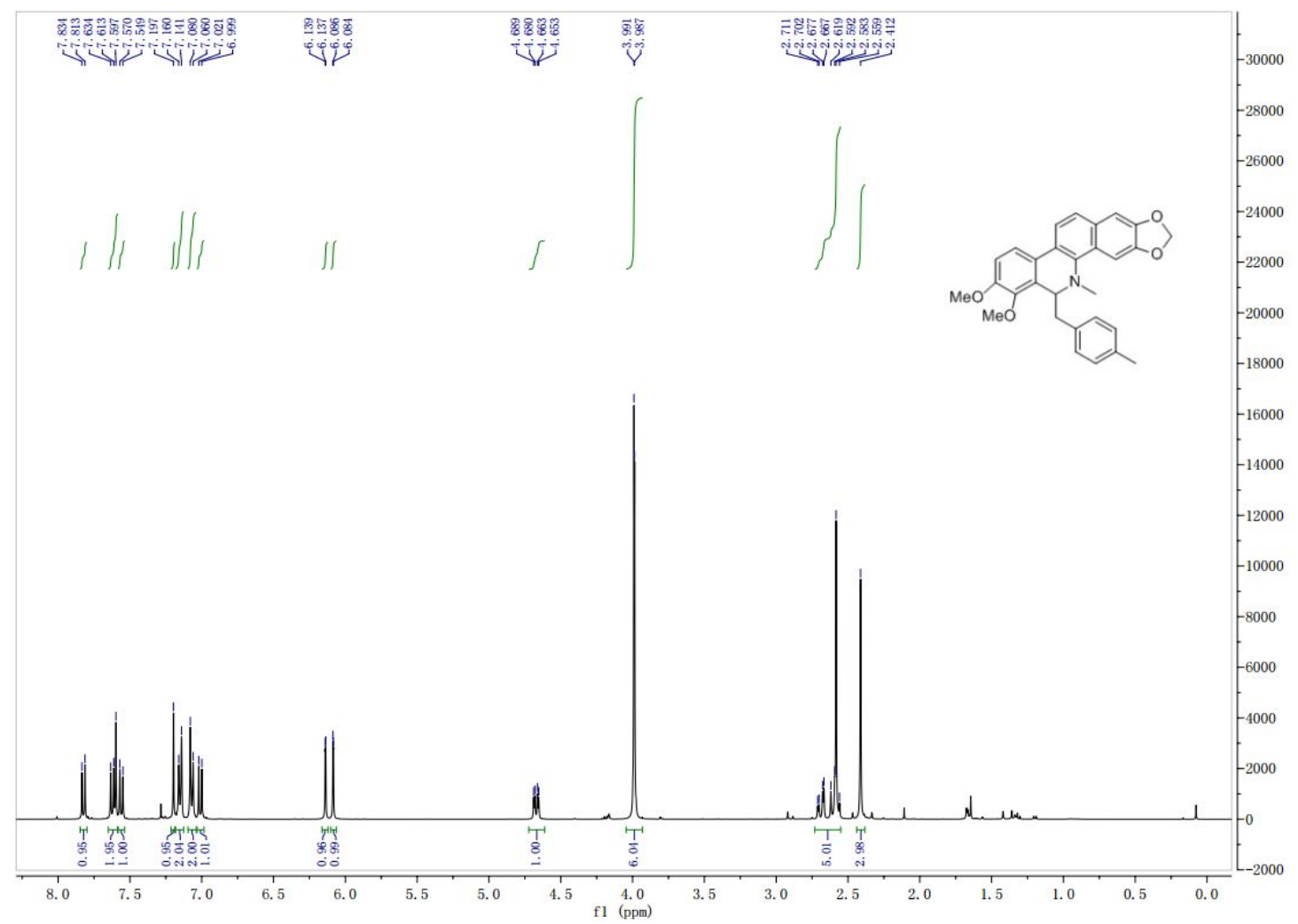

Figure S11. ${ }^{1} \mathrm{H}$ NMR spectrum $\left(400 \mathrm{MHz}, \mathrm{CDCl}_{3}\right)$ of $\mathbf{7 b b}$

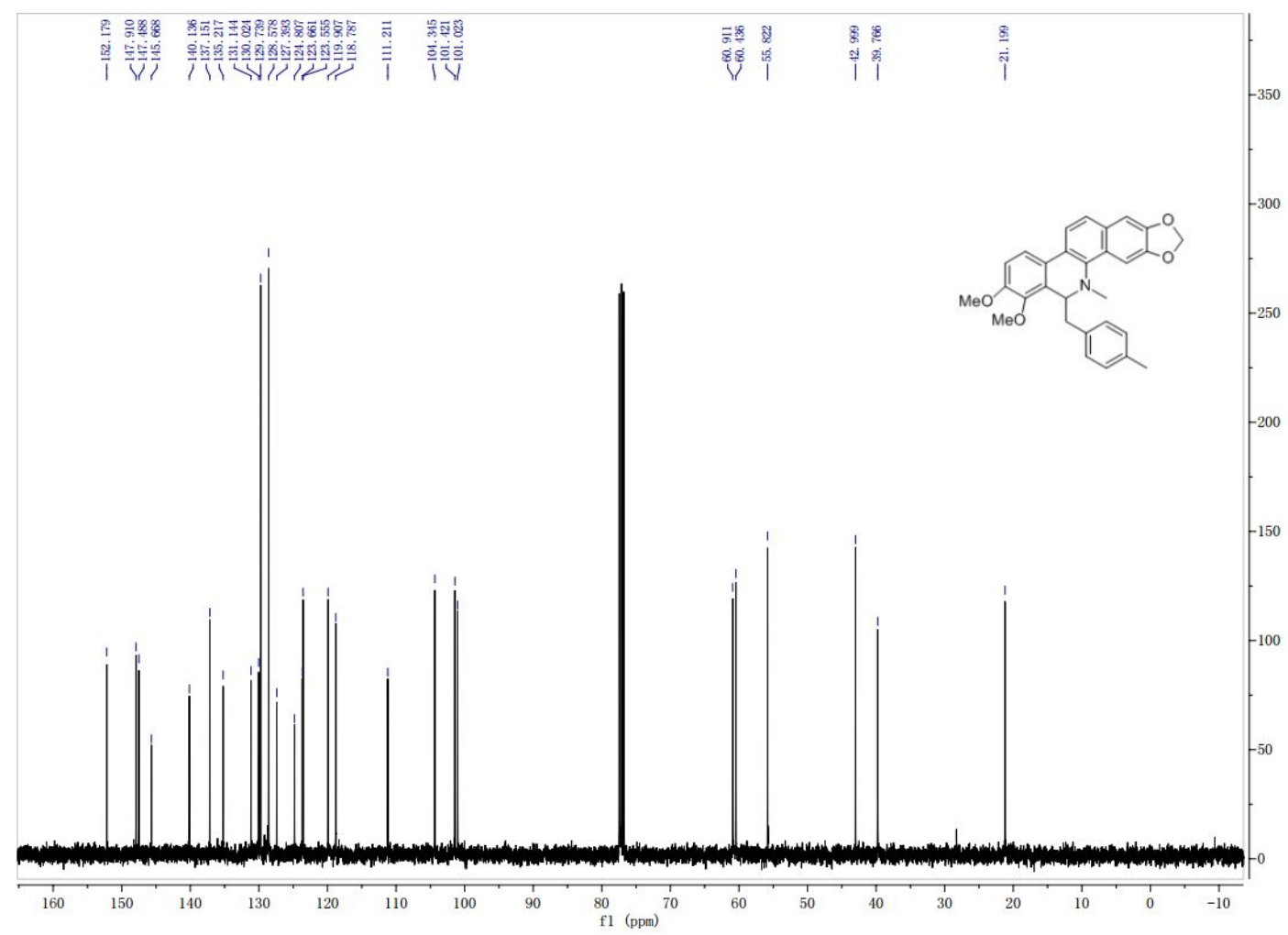

Figure S12. ${ }^{13} \mathrm{C}$ NMR spectrum $\left(400 \mathrm{MHz}, \mathrm{CDCl}_{3}\right)$ of $\mathbf{7 b b}$ 


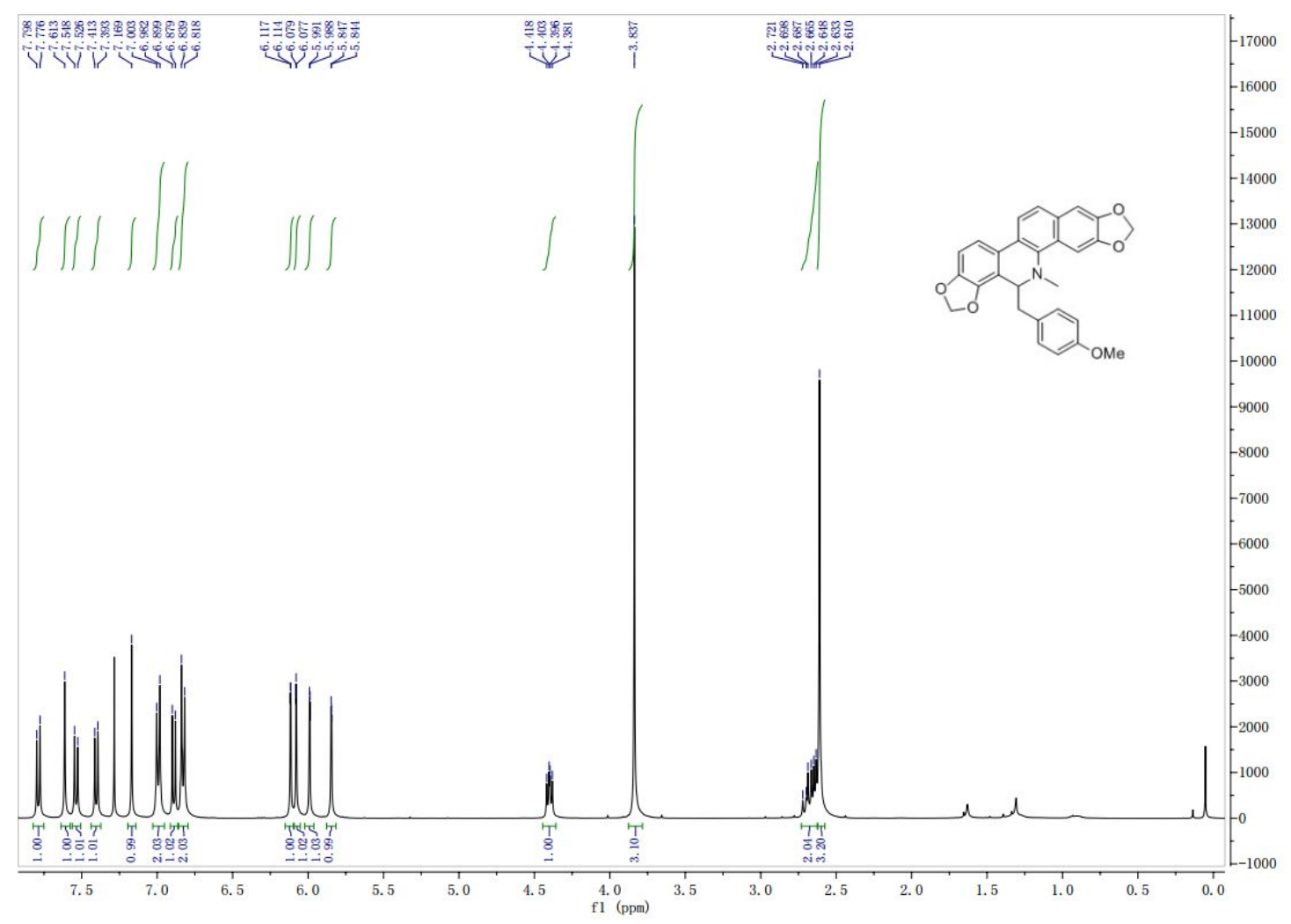

Figure S13. ${ }^{1} \mathrm{H}$ NMR spectrum $\left(400 \mathrm{MHz}, \mathrm{CDCl}_{3}\right)$ of $7 \mathbf{c}$

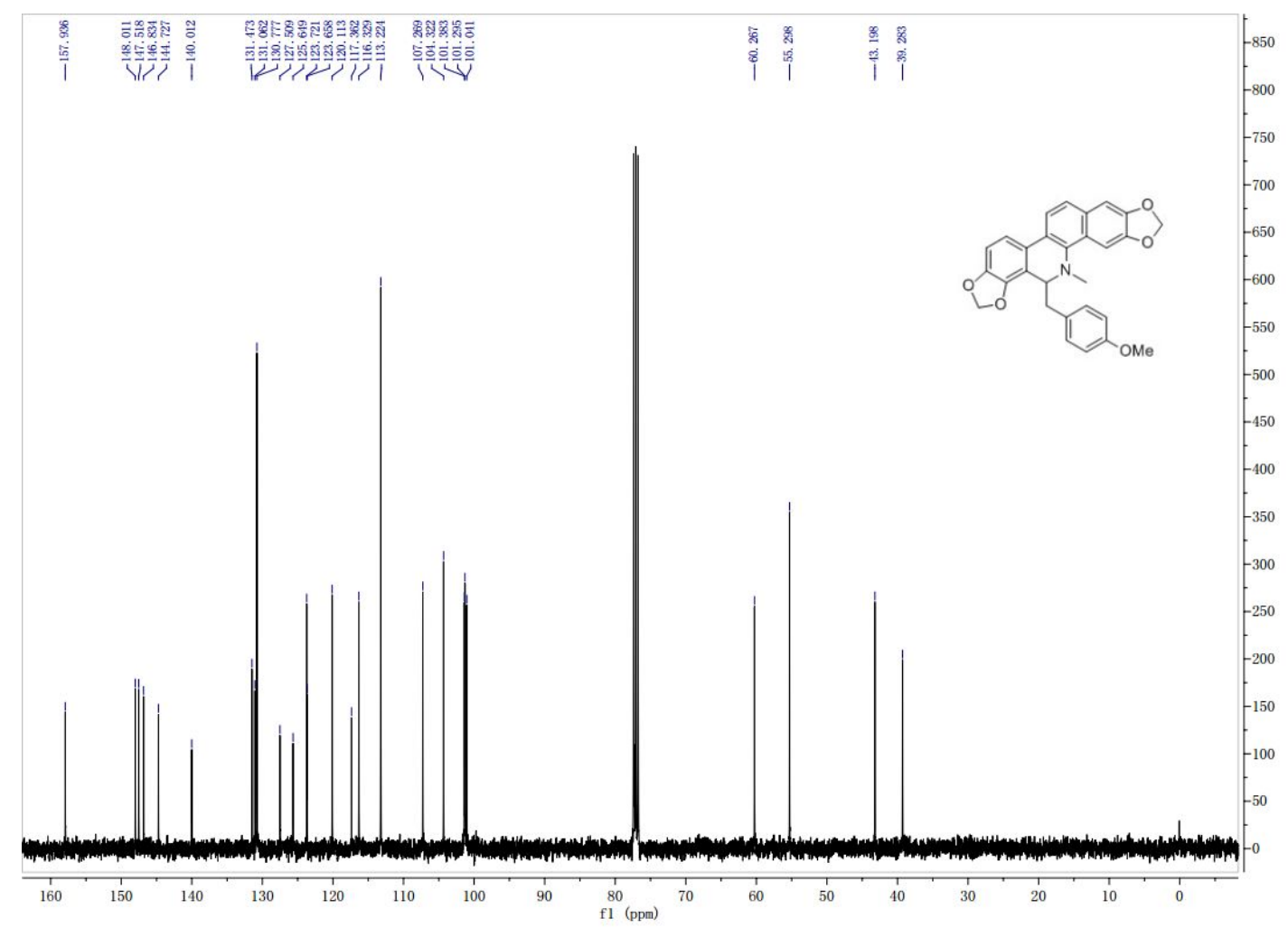

Figure $\mathbf{S 1 4} .{ }^{13} \mathrm{C}$ NMR spectrum $\left(400 \mathrm{MHz}, \mathrm{CDCl}_{3}\right)$ of $\mathbf{7 c}$ 


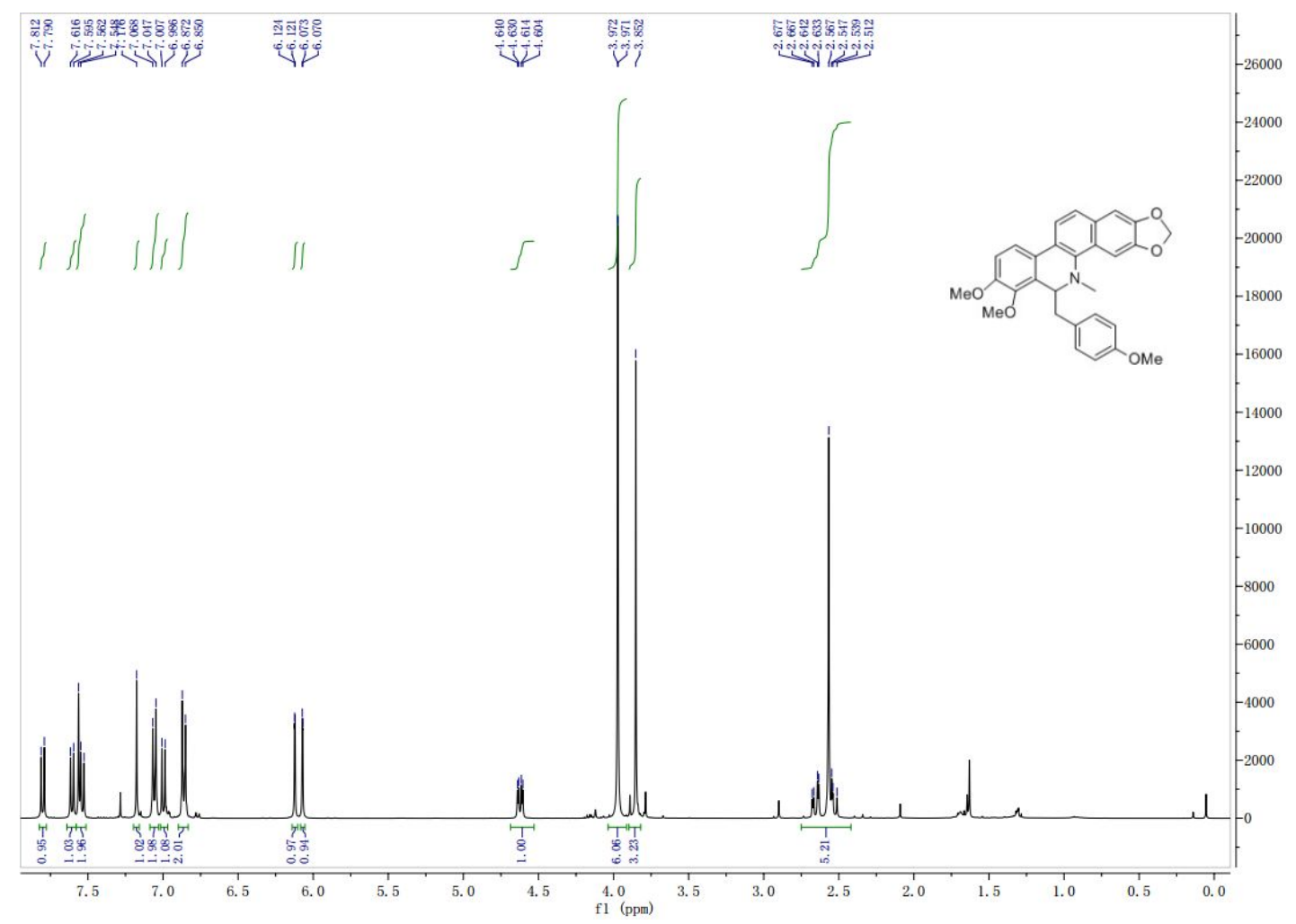

Figure S15. ${ }^{1} \mathrm{H}$ NMR spectrum $\left(400 \mathrm{MHz}, \mathrm{CDCl}_{3}\right)$ of $7 \mathbf{c c}$

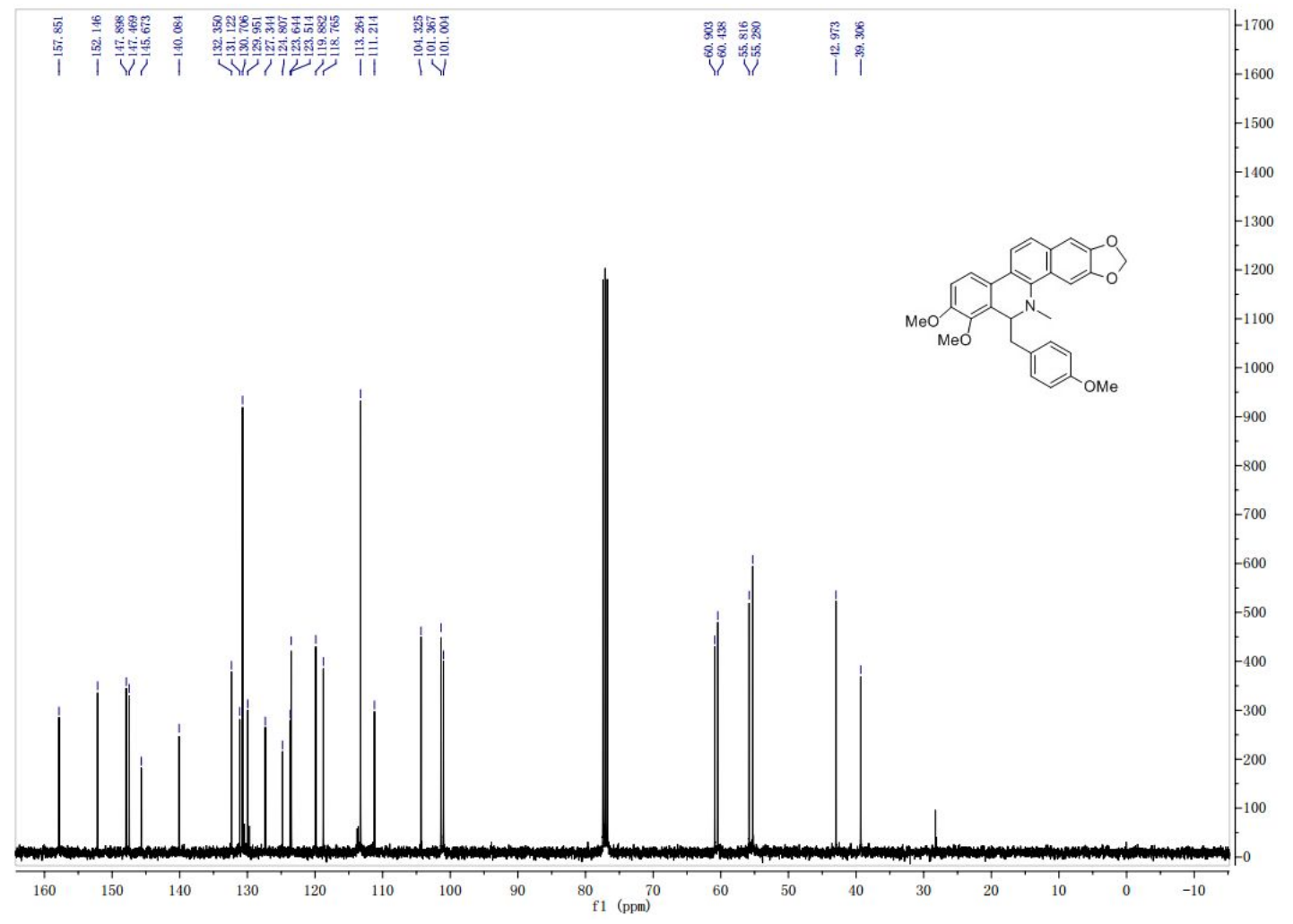

Figure S16. ${ }^{13} \mathrm{C} \mathrm{NMR}$ spectrum $\left(400 \mathrm{MHz}, \mathrm{CDCl}_{3}\right)$ of $7 \mathbf{c c}$ 


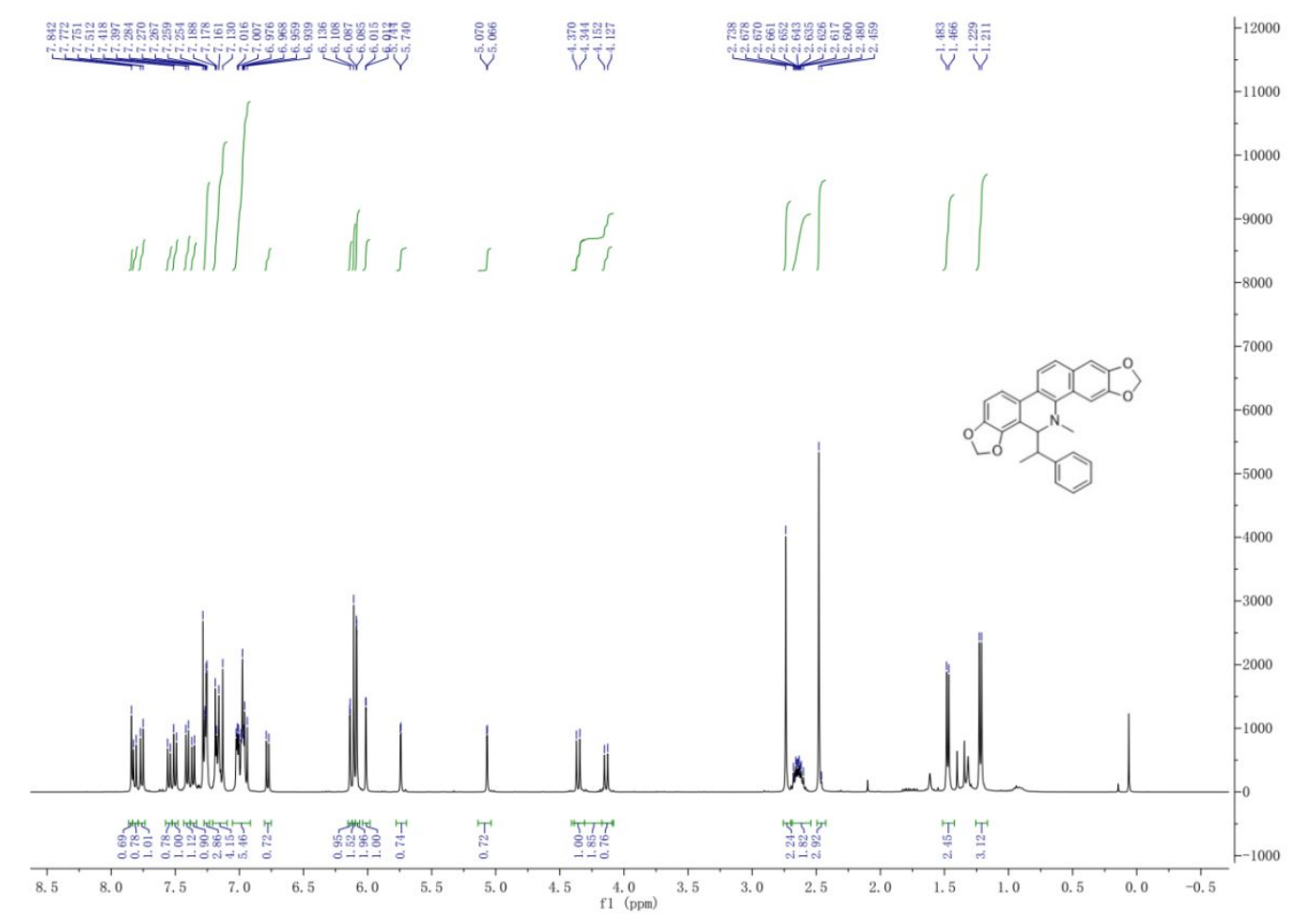

Figure S17. ${ }^{1} \mathrm{H}$ NMR spectrum $\left(400 \mathrm{MHz}, \mathrm{CDCl}_{3}\right)$ of $7 \mathbf{d}$

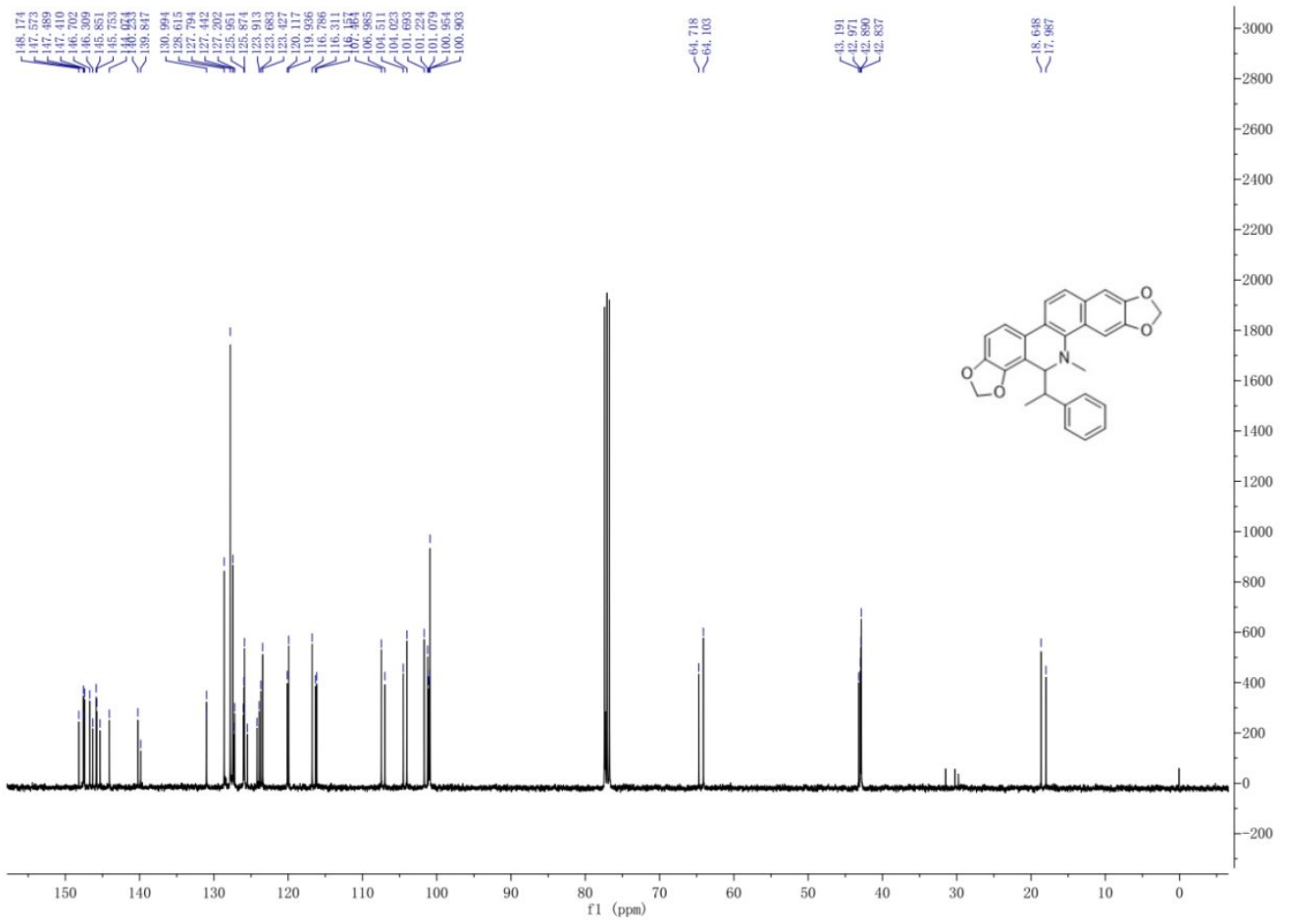

Figure S18. ${ }^{13} \mathrm{C}$ NMR spectrum $\left(400 \mathrm{MHz}, \mathrm{CDCl}_{3}\right)$ of $\mathbf{7 d}$ 


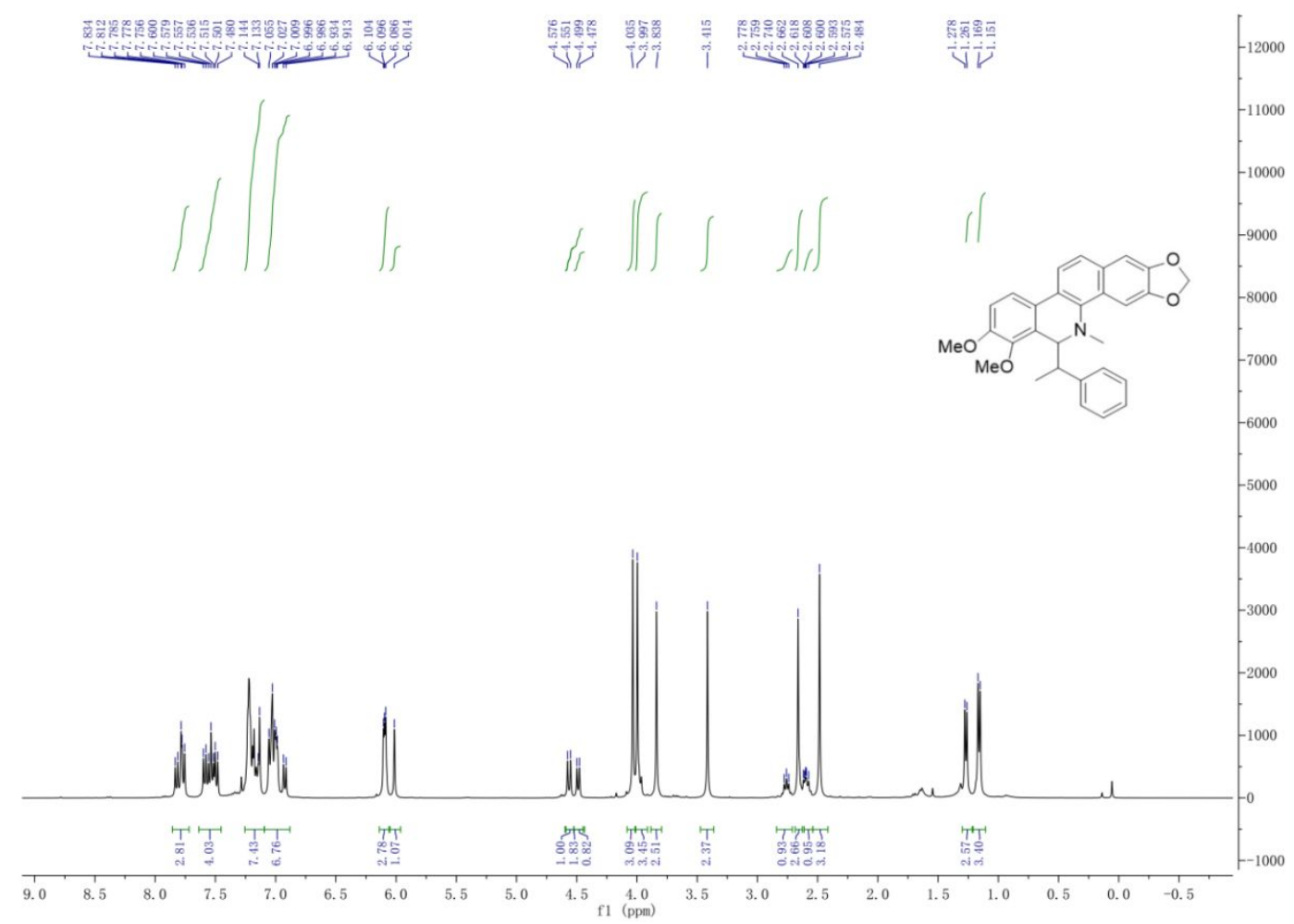

Figure S19. ${ }^{1} \mathrm{H}$ NMR spectrum $\left(400 \mathrm{MHz}, \mathrm{CDCl}_{3}\right)$ of $\mathbf{7 d d}$

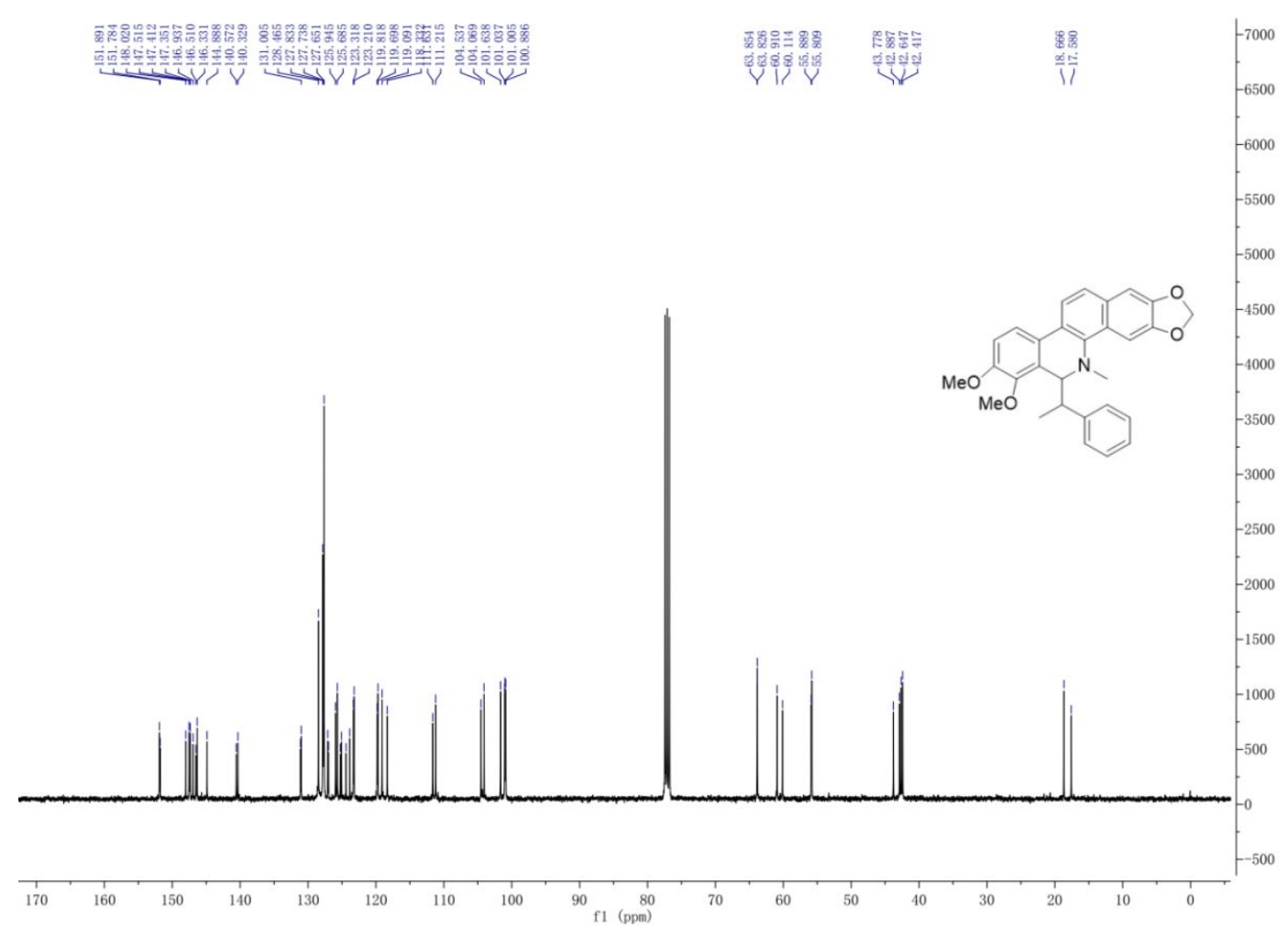

Figure S20. ${ }^{13} \mathrm{C}$ NMR spectrum $\left(400 \mathrm{MHz}, \mathrm{CDCl}_{3}\right)$ of 7dd 


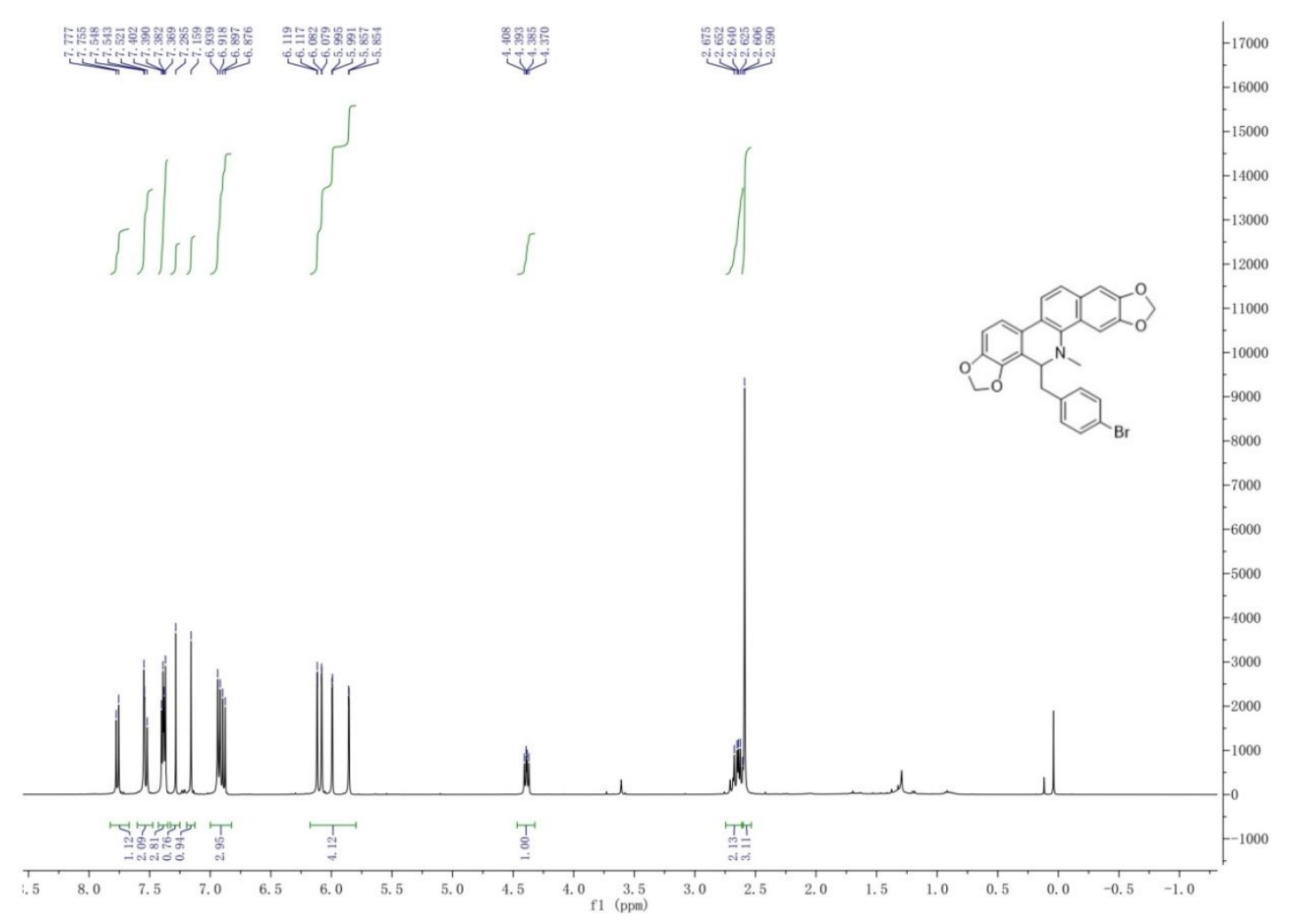

Figure S21. ${ }^{1} \mathrm{H}$ NMR spectrum $\left(400 \mathrm{MHz}, \mathrm{CDCl}_{3}\right)$ of $\mathbf{7 e}$

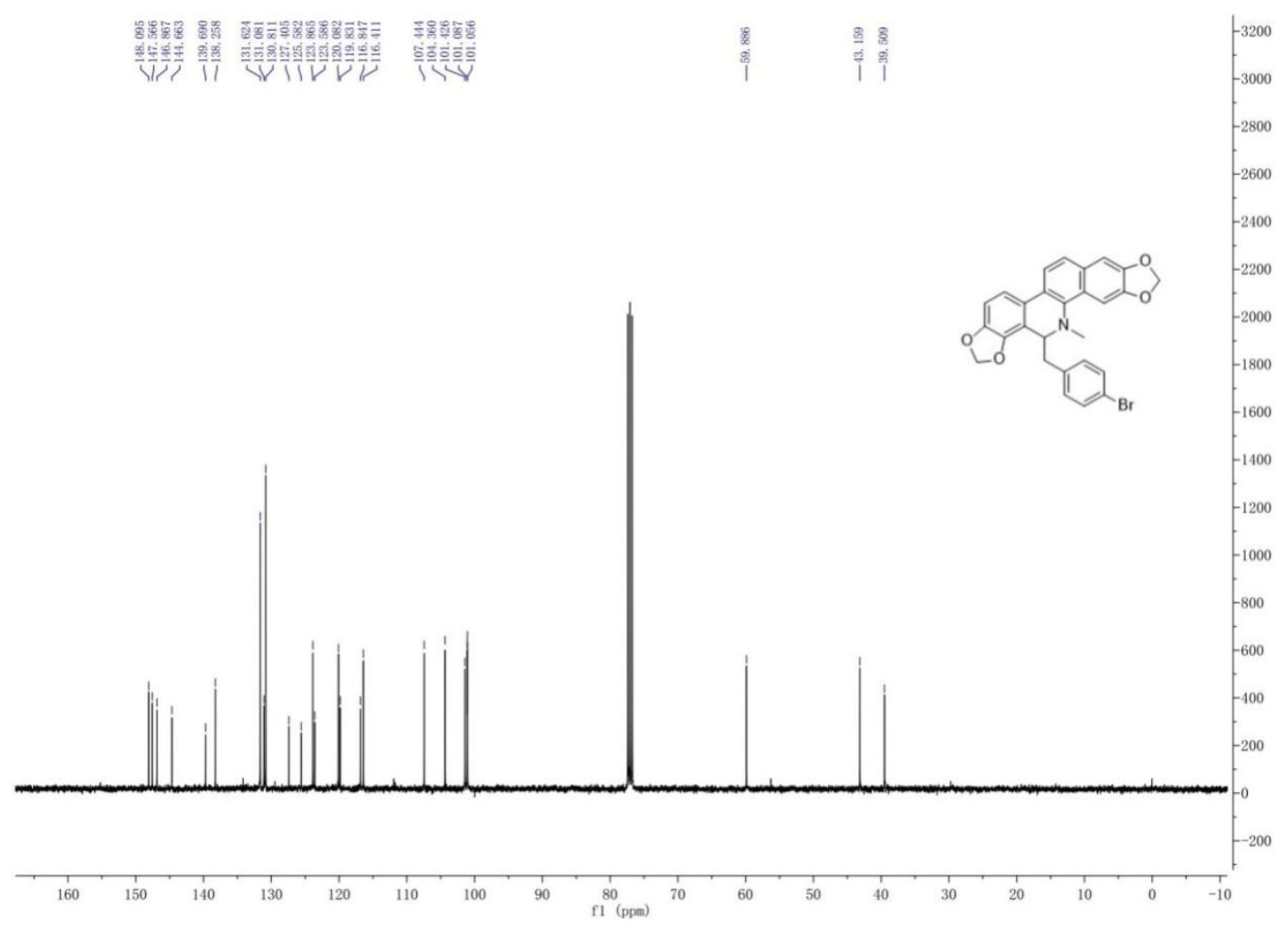

Figure S22. ${ }^{13} \mathrm{C}$ NMR spectrum $\left(400 \mathrm{MHz}, \mathrm{CDCl}_{3}\right)$ of $7 \mathbf{e}$ 


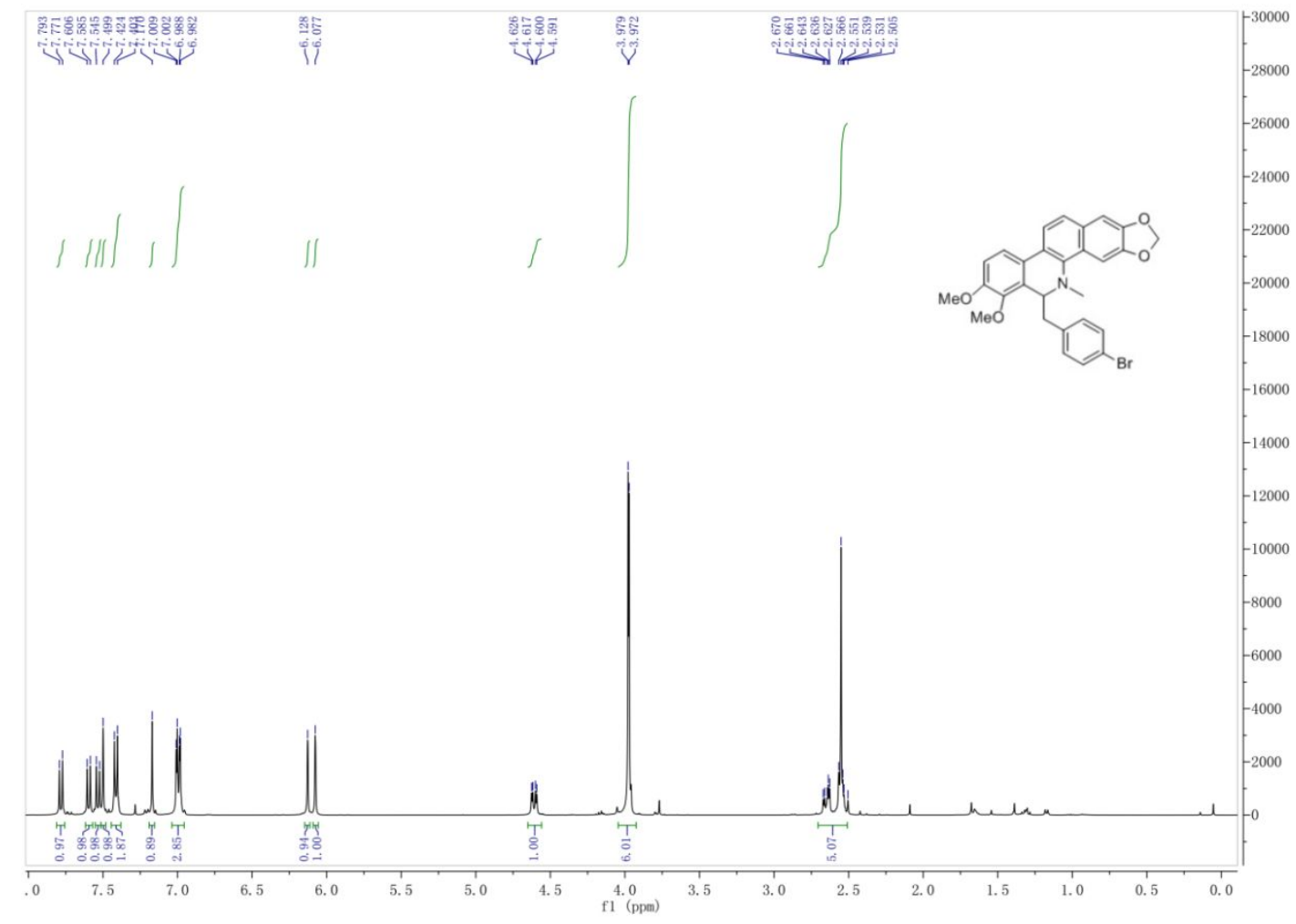

Figure S23. ${ }^{1} \mathrm{H}$ NMR spectrum $\left(400 \mathrm{MHz}, \mathrm{CDCl}_{3}\right)$ of 7ee

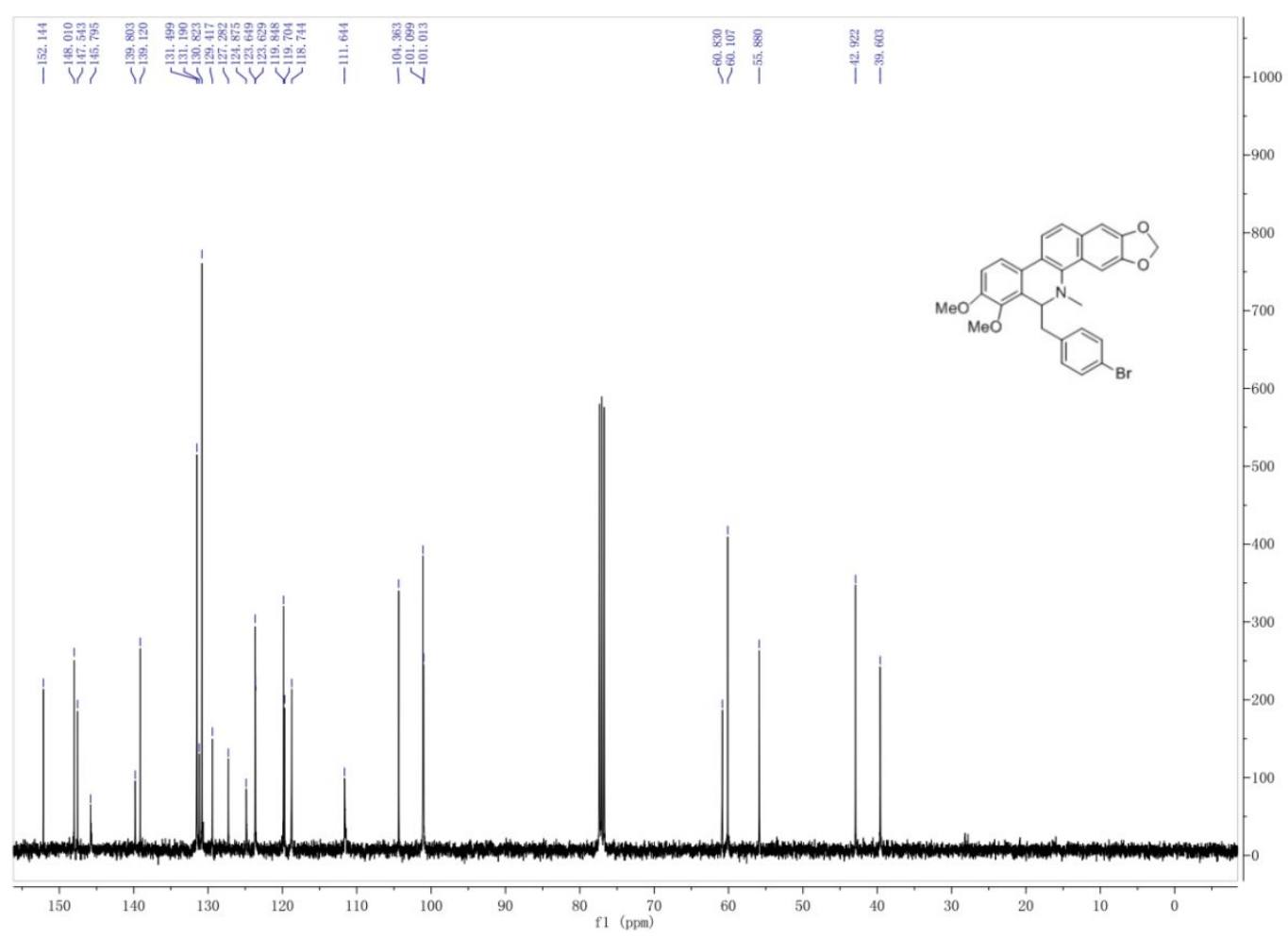

Figure S24. ${ }^{13} \mathrm{C}$ NMR spectrum $\left(400 \mathrm{MHz}, \mathrm{CDCl}_{3}\right)$ of 7ee 


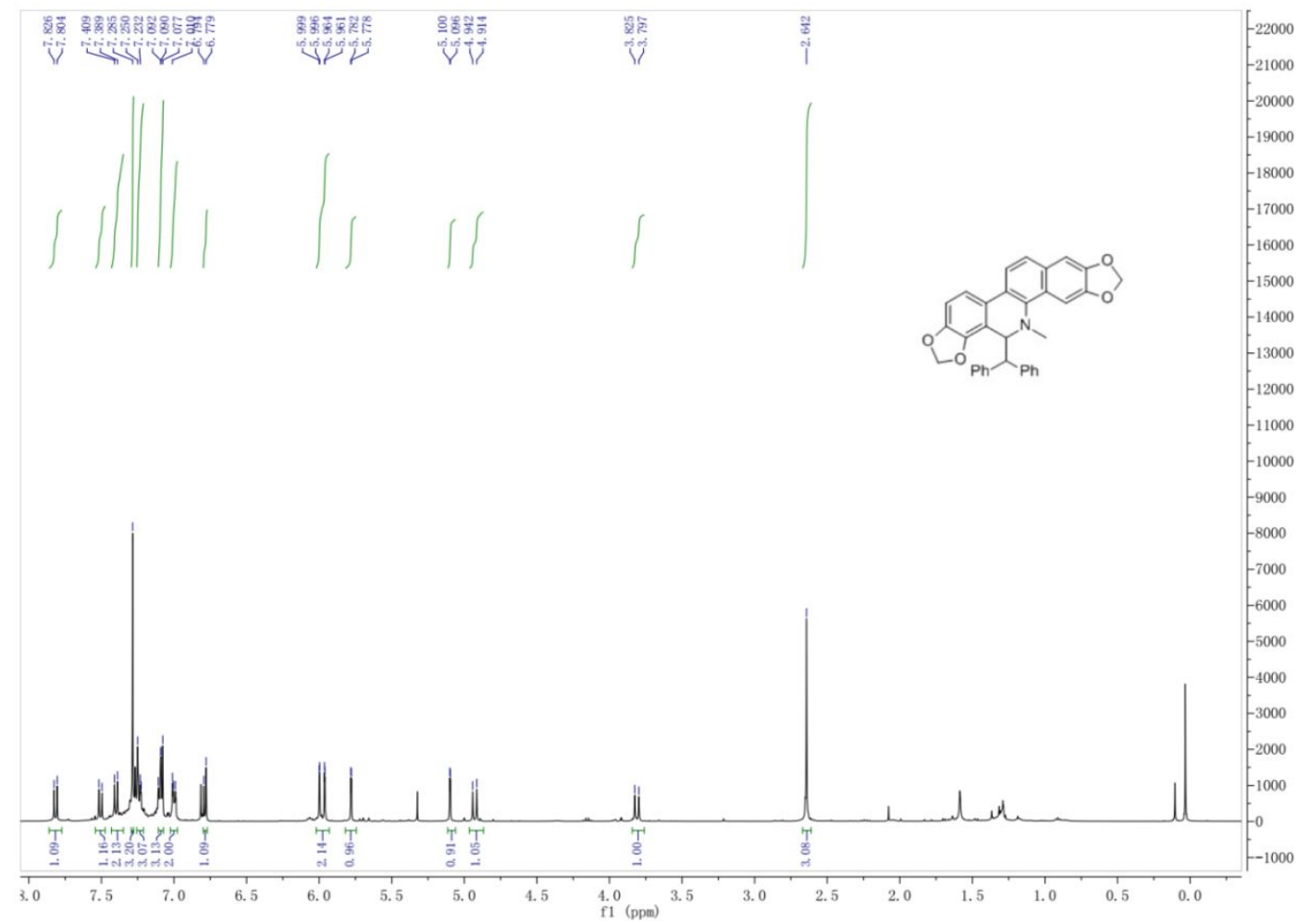

Figure S25. ${ }^{1} \mathrm{H}$ NMR spectrum $\left(400 \mathrm{MHz}, \mathrm{CDCl}_{3}\right)$ of $7 \mathbf{f}$

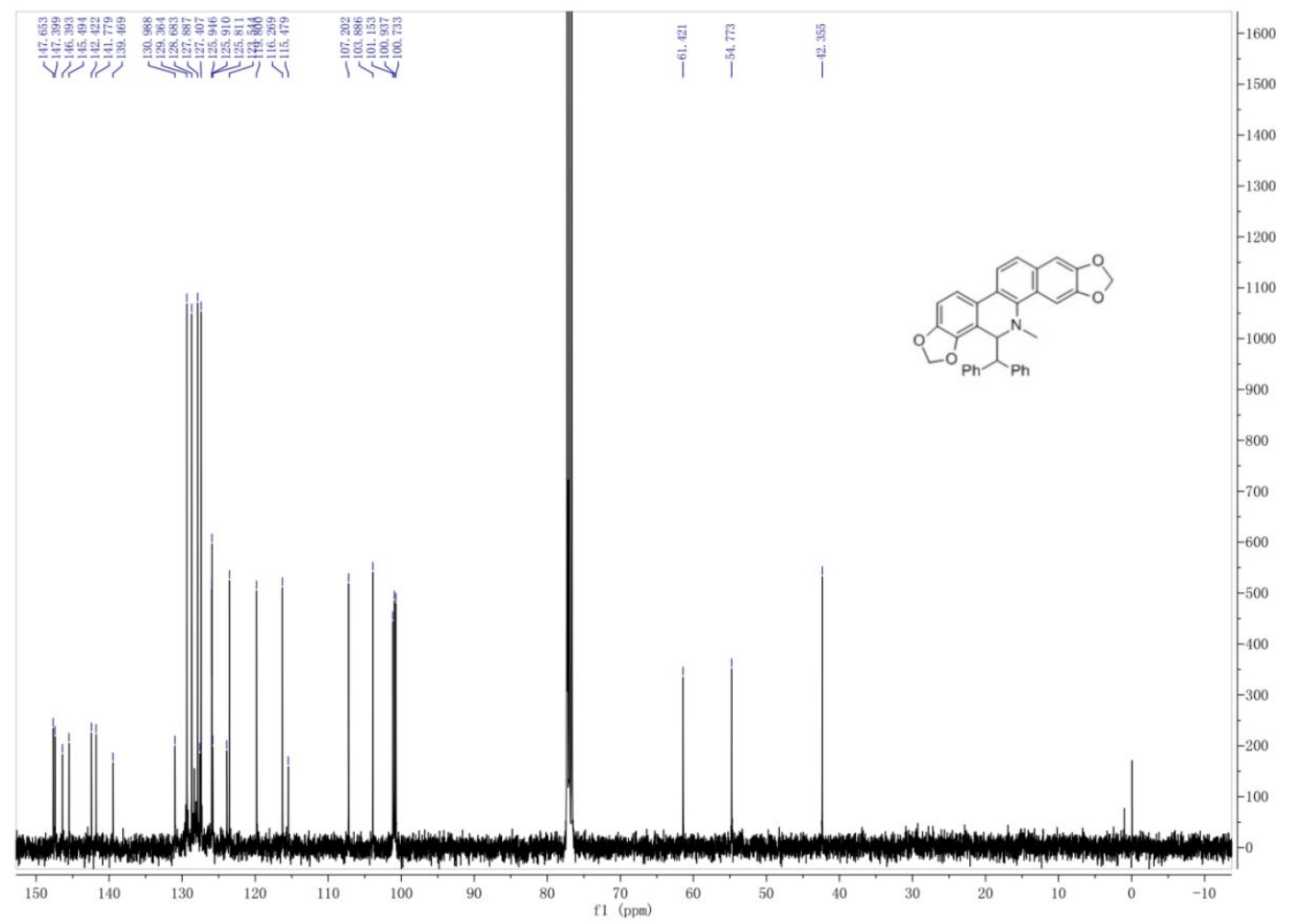

Figure S26. ${ }^{13} \mathrm{C}$ NMR spectrum $\left(400 \mathrm{MHz}, \mathrm{CDCl}_{3}\right)$ of $\mathbf{7 f}$ 


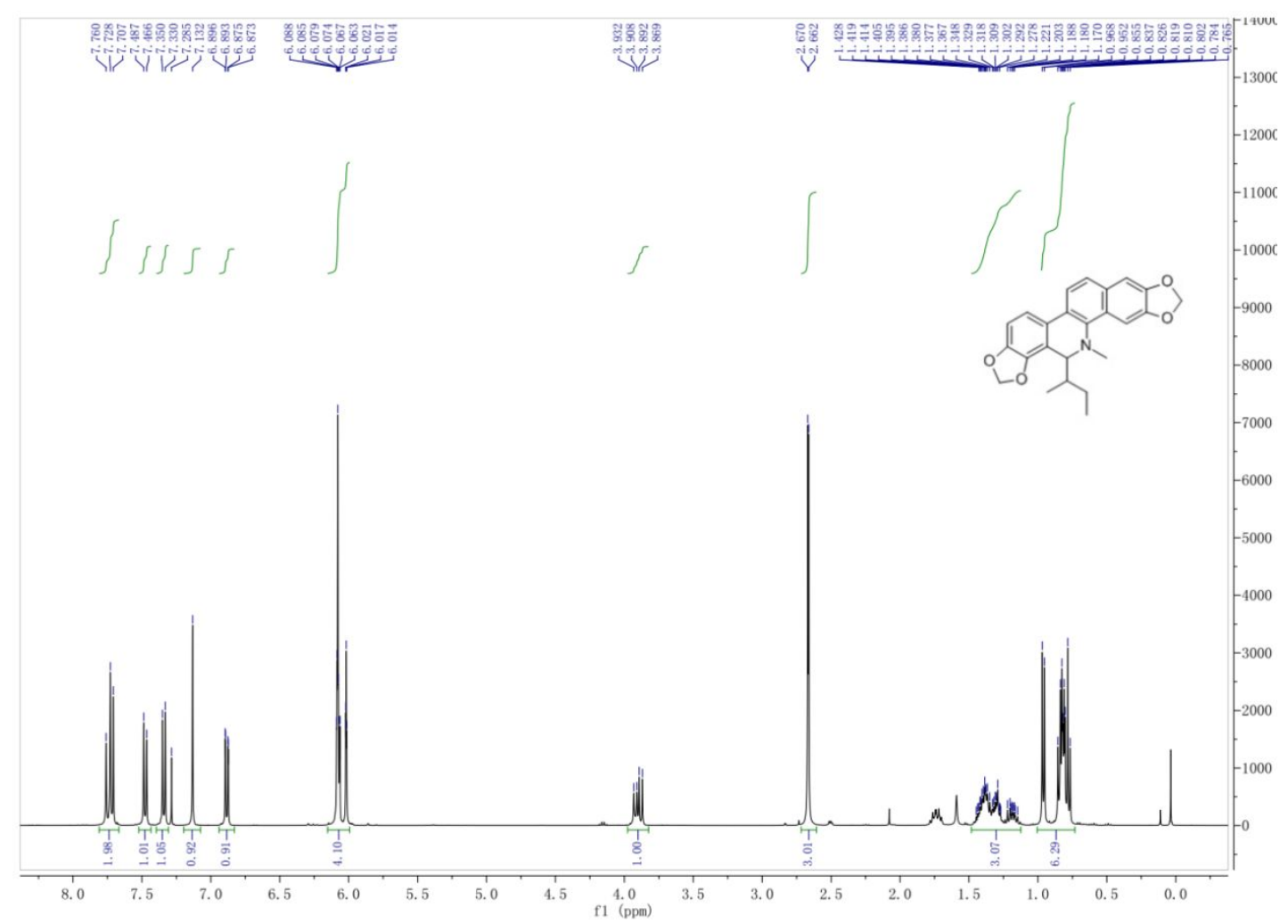

Figure S27. ${ }^{1} \mathrm{H}$ NMR spectrum $\left(400 \mathrm{MHz}, \mathrm{CDCl}_{3}\right)$ of $\mathbf{7 g}$

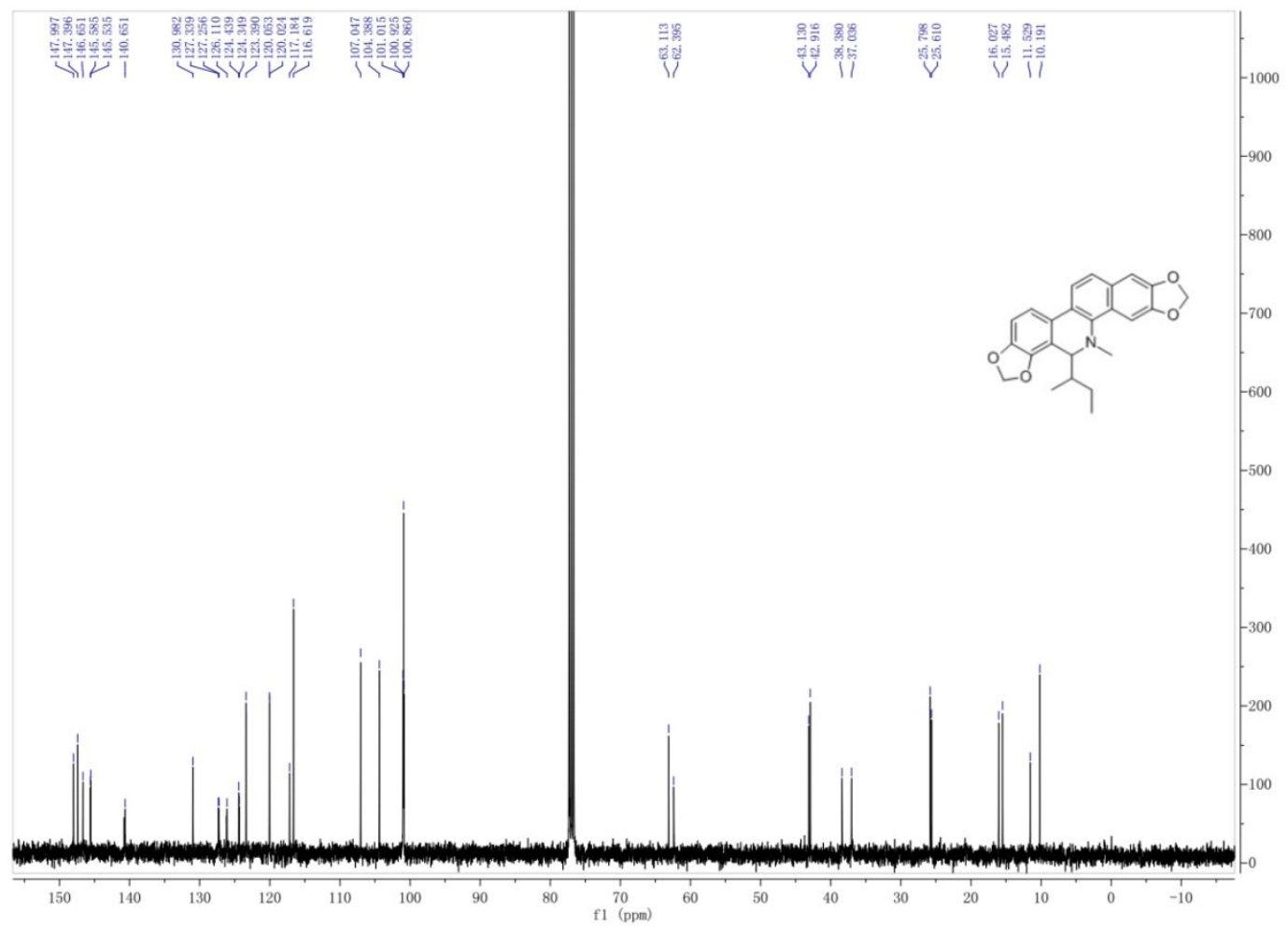

Figure S28. ${ }^{13} \mathrm{C}$ NMR spectrum $\left(400 \mathrm{MHz}, \mathrm{CDCl}_{3}\right)$ of $\mathbf{7 g}$ 


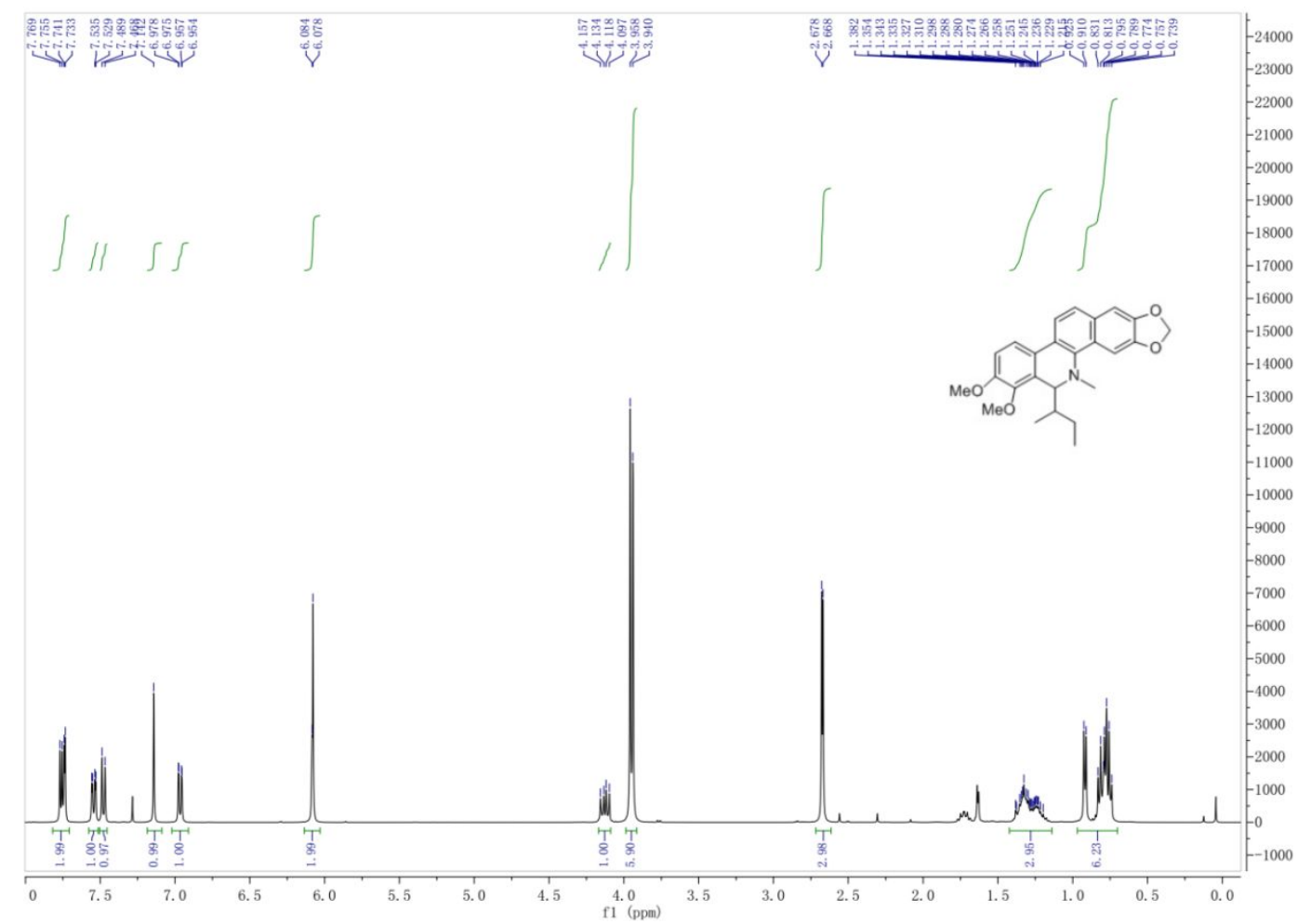

Figure S29. ${ }^{1} \mathrm{H}$ NMR spectrum $\left(400 \mathrm{MHz}, \mathrm{CDCl}_{3}\right)$ of $7 \mathbf{g g}$

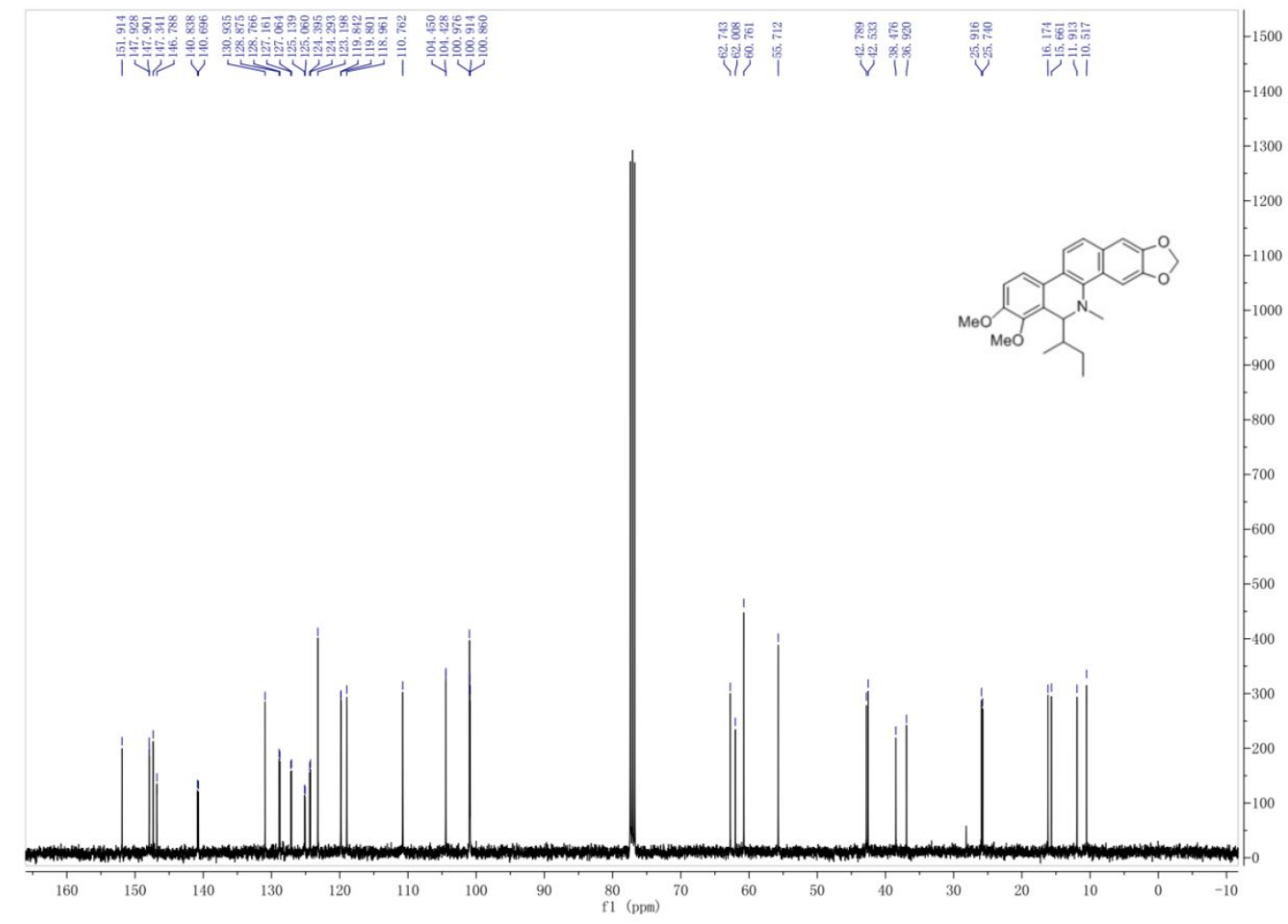

Figure S30. ${ }^{13} \mathrm{C}$ NMR spectrum $\left(400 \mathrm{MHz}, \mathrm{CDCl}_{3}\right)$ of $\mathbf{7 g g}$ 


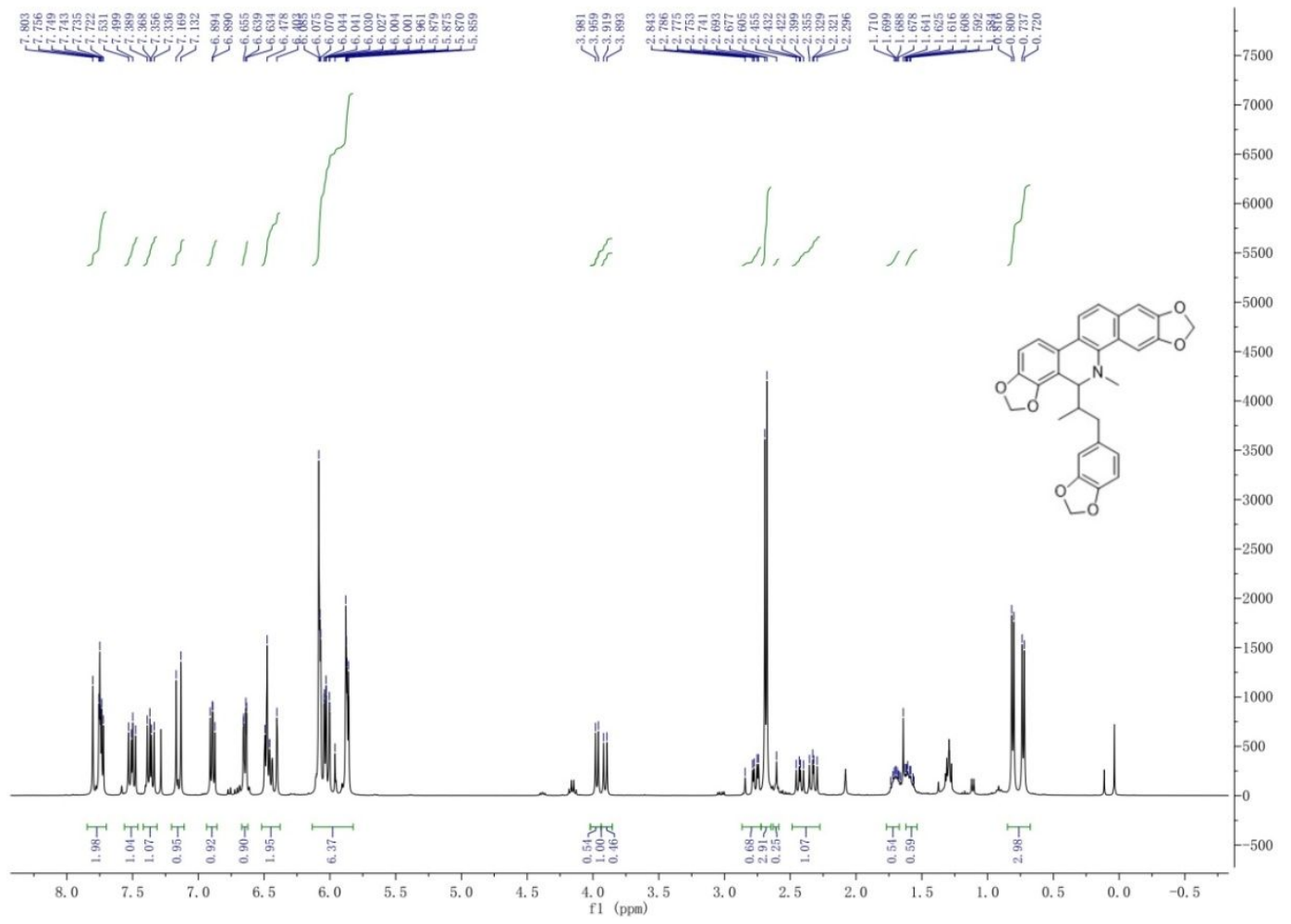

Figure S31. ${ }^{1} \mathrm{H}$ NMR spectrum $\left(400 \mathrm{MHz}, \mathrm{CDCl}_{3}\right)$ of $\mathbf{7 h}$

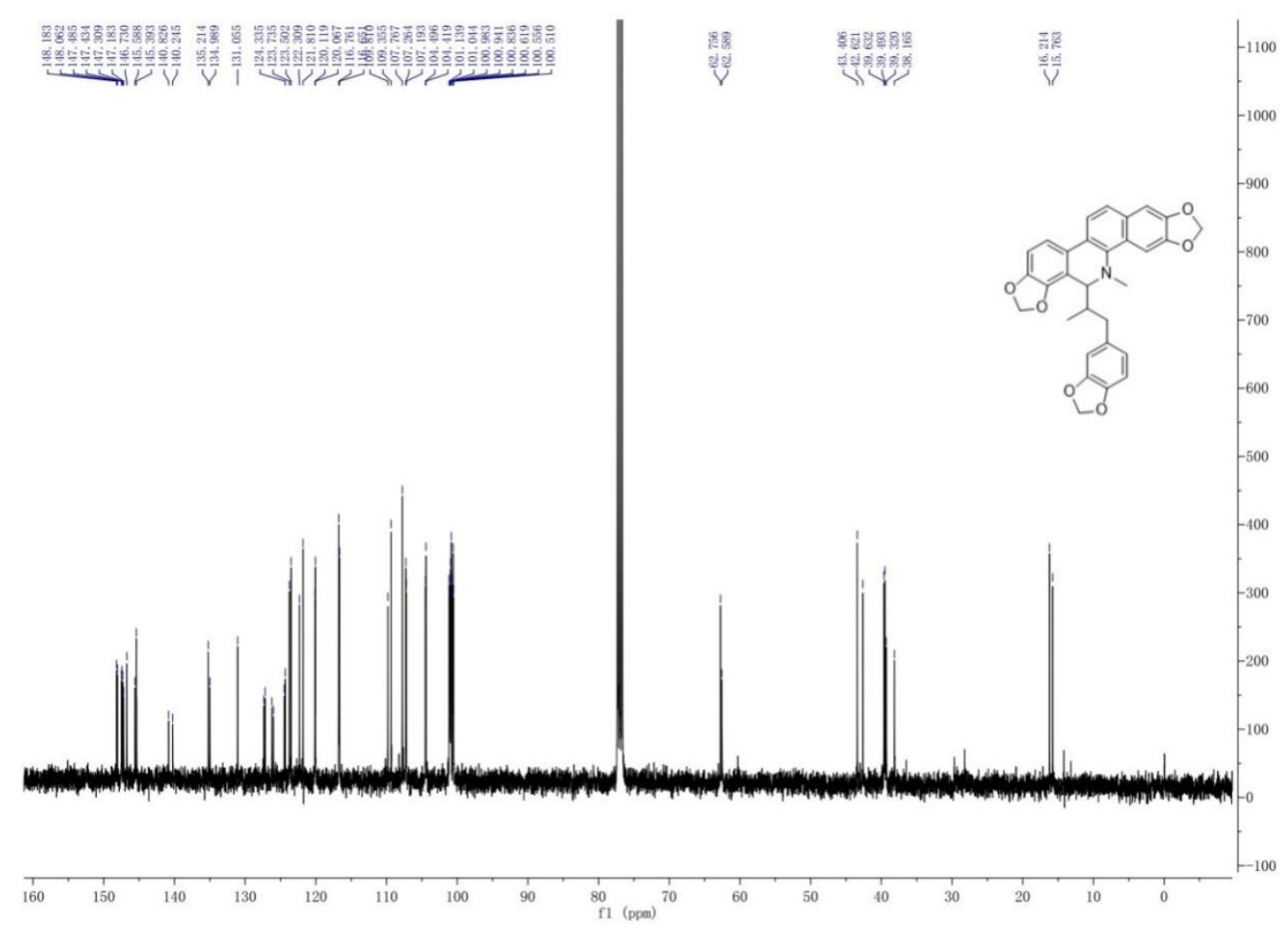

Figure S32. ${ }^{13} \mathrm{C}$ NMR spectrum $\left(400 \mathrm{MHz}, \mathrm{CDCl}_{3}\right)$ of $\mathbf{7 h}$ 


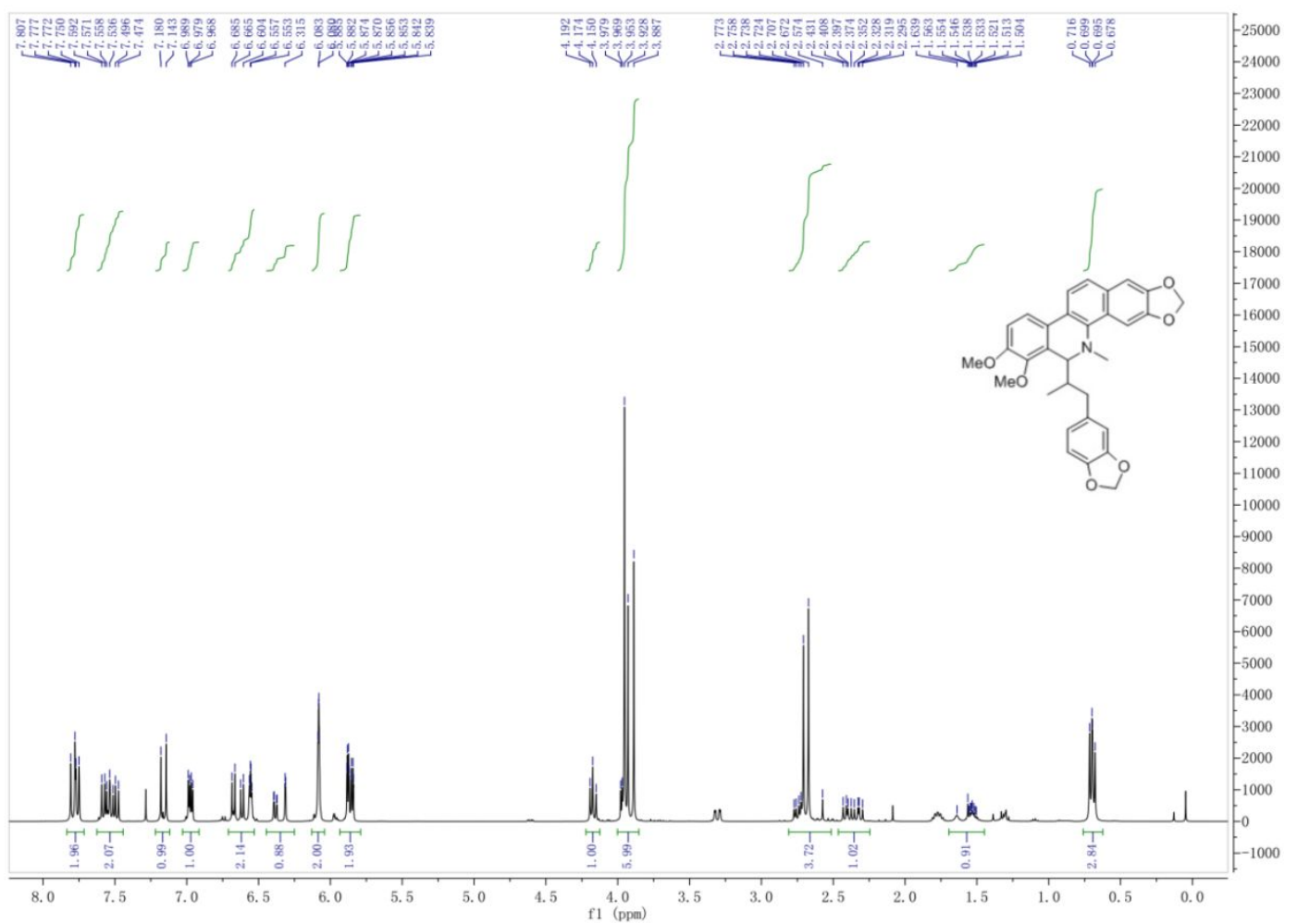

Figure S33. ${ }^{1} \mathrm{H}$ NMR spectrum $\left(400 \mathrm{MHz}, \mathrm{CDCl}_{3}\right)$ of $\mathbf{7 h h}$

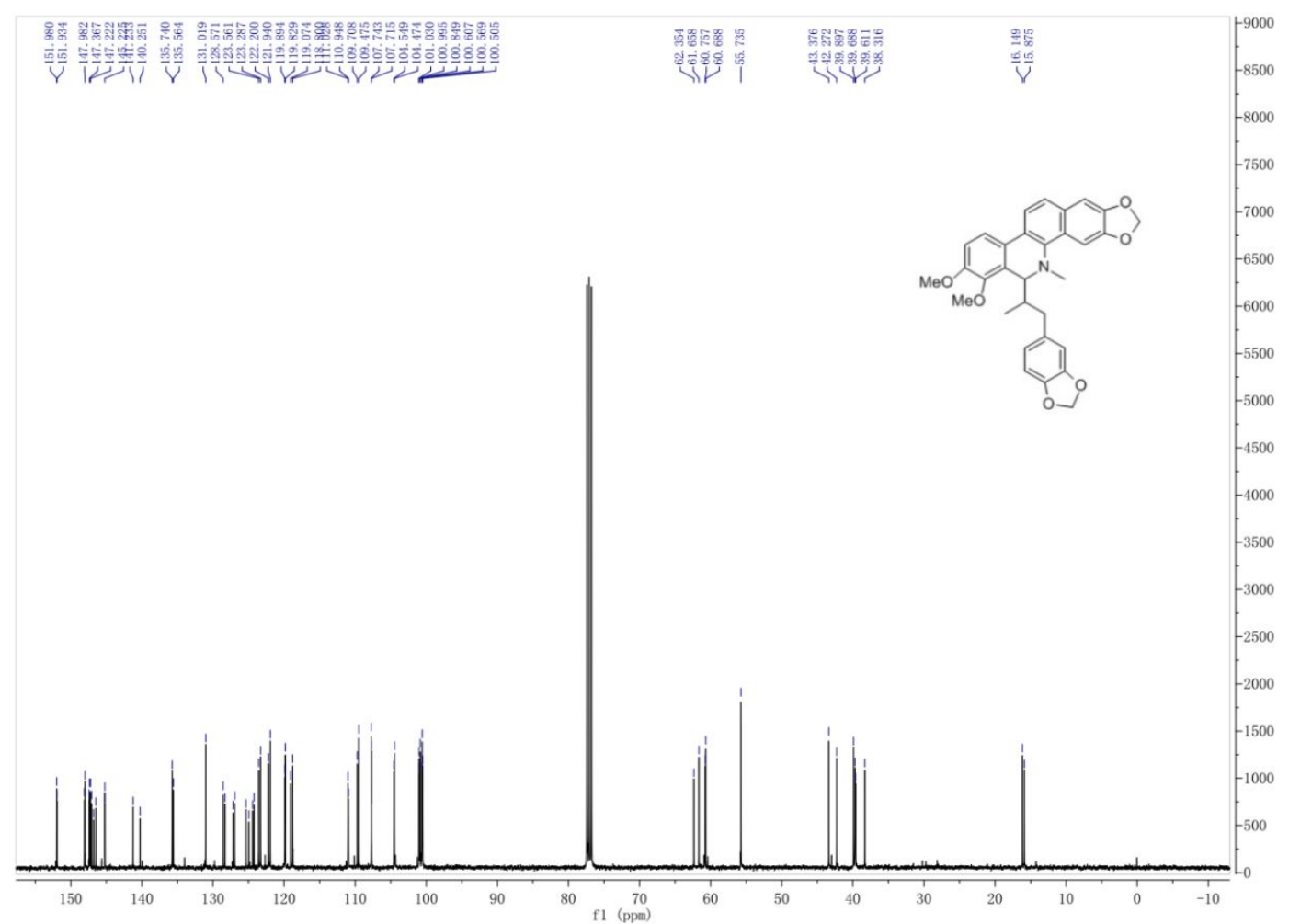

Figure S34. ${ }^{13} \mathrm{C}$ NMR spectrum $\left(400 \mathrm{MHz}, \mathrm{CDCl}_{3}\right)$ of $\mathbf{7 h h}$ 


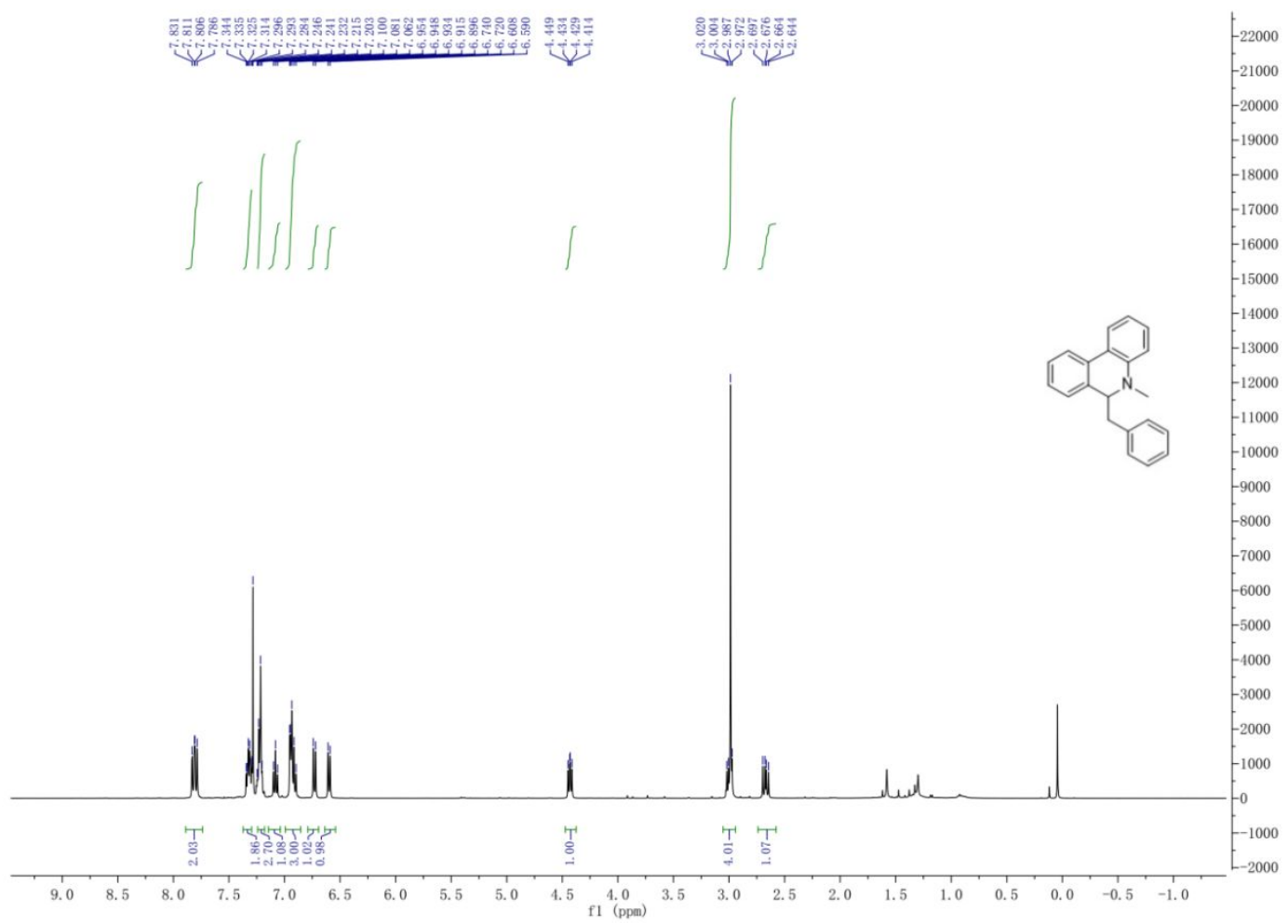

Figure S35. ${ }^{1} \mathrm{H}$ NMR spectrum $\left(400 \mathrm{MHz}, \mathrm{CDCl}_{3}\right)$ of $\mathbf{1 2}$

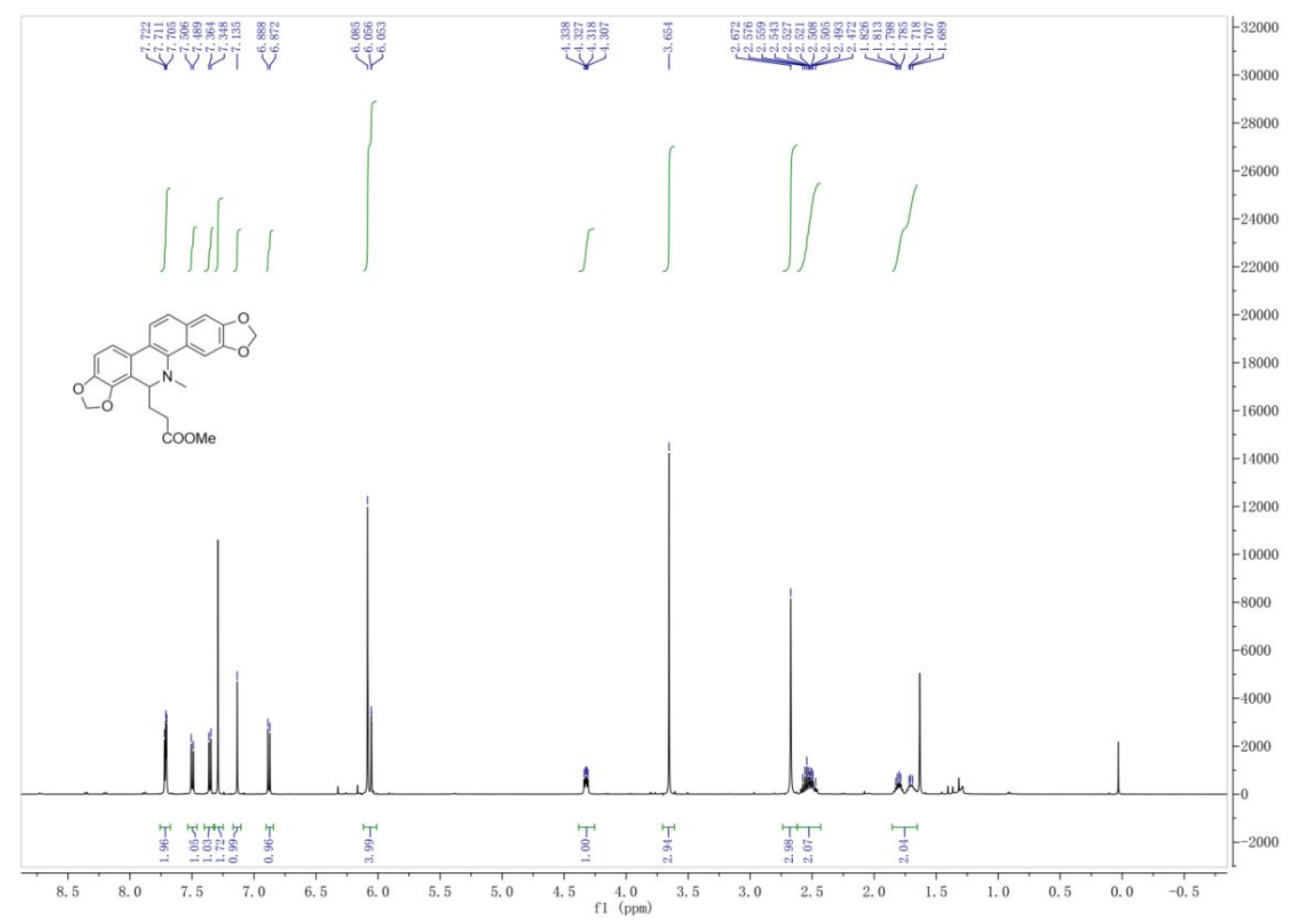

Figure S36. ${ }^{1} \mathrm{H}$ NMR spectrum $\left(400 \mathrm{MHz}, \mathrm{CDCl}_{3}\right)$ of $\mathbf{1 6 a}$ 


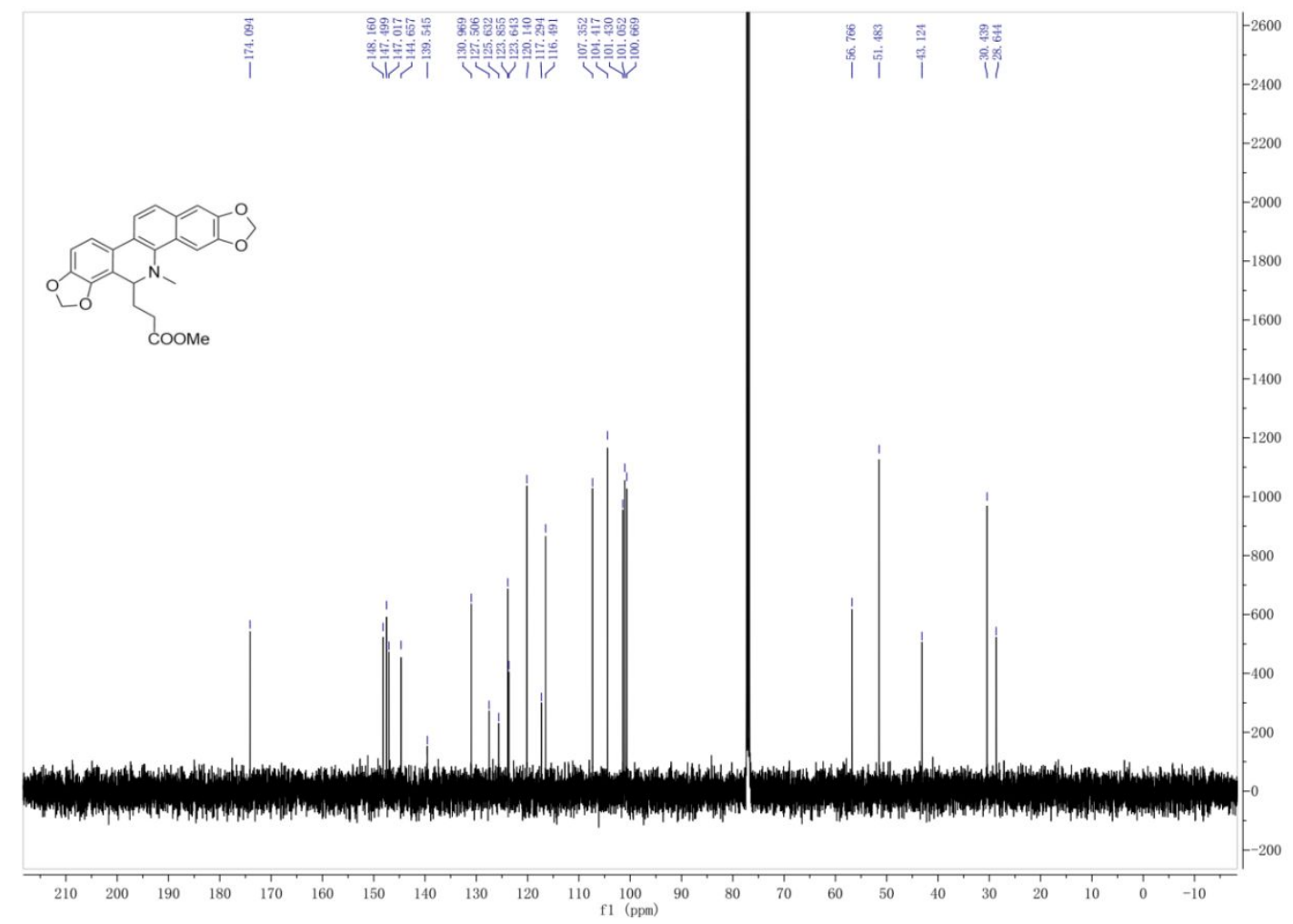

Figure S37. ${ }^{13} \mathrm{C}$ NMR spectrum $\left(400 \mathrm{MHz}, \mathrm{CDCl}_{3}\right)$ of $\mathbf{1 6 a}$

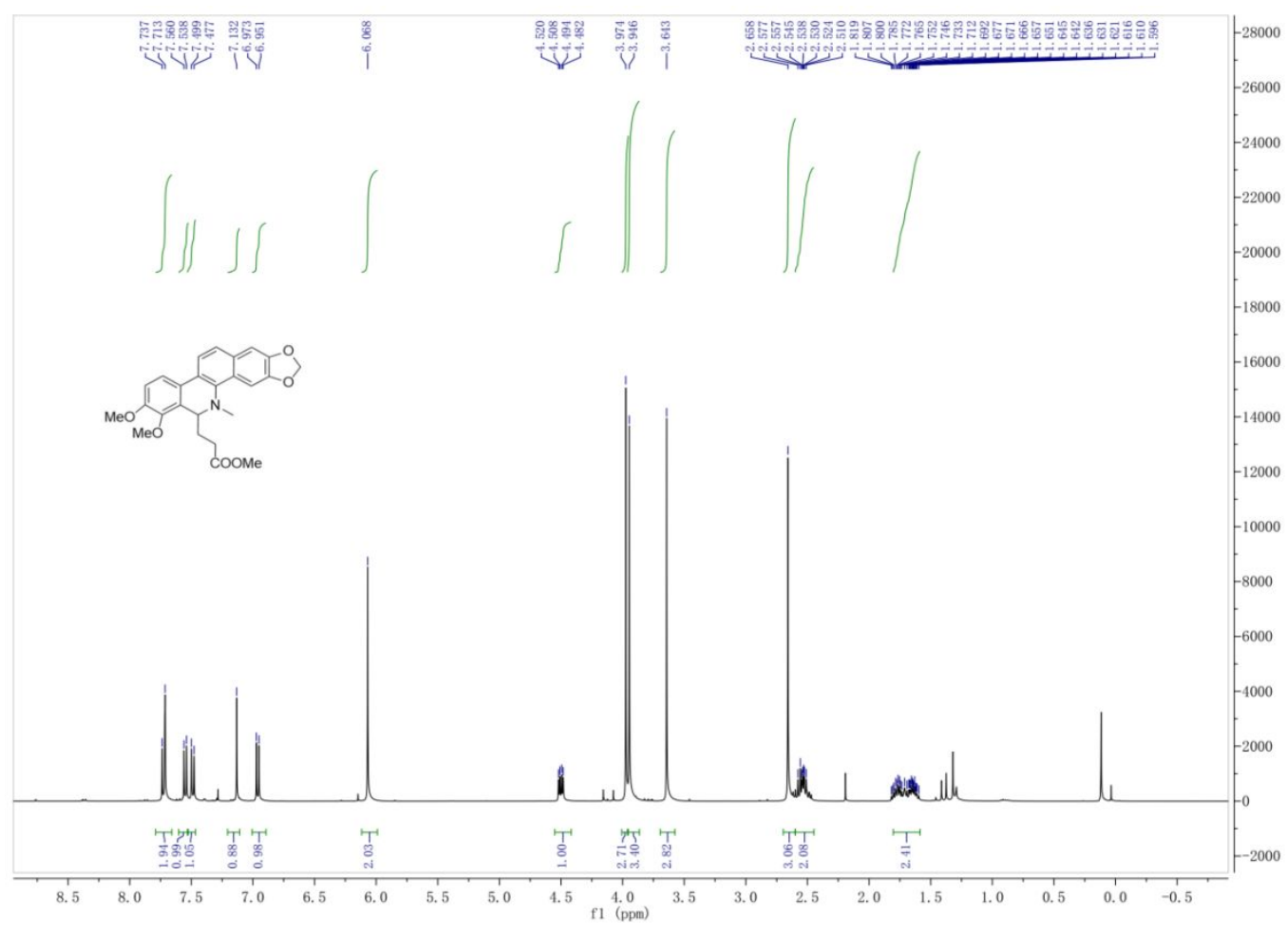

Figure S38. ${ }^{1} \mathrm{H} \mathrm{NMR}$ spectrum $\left(400 \mathrm{MHz}, \mathrm{CDCl}_{3}\right)$ of 16aa 


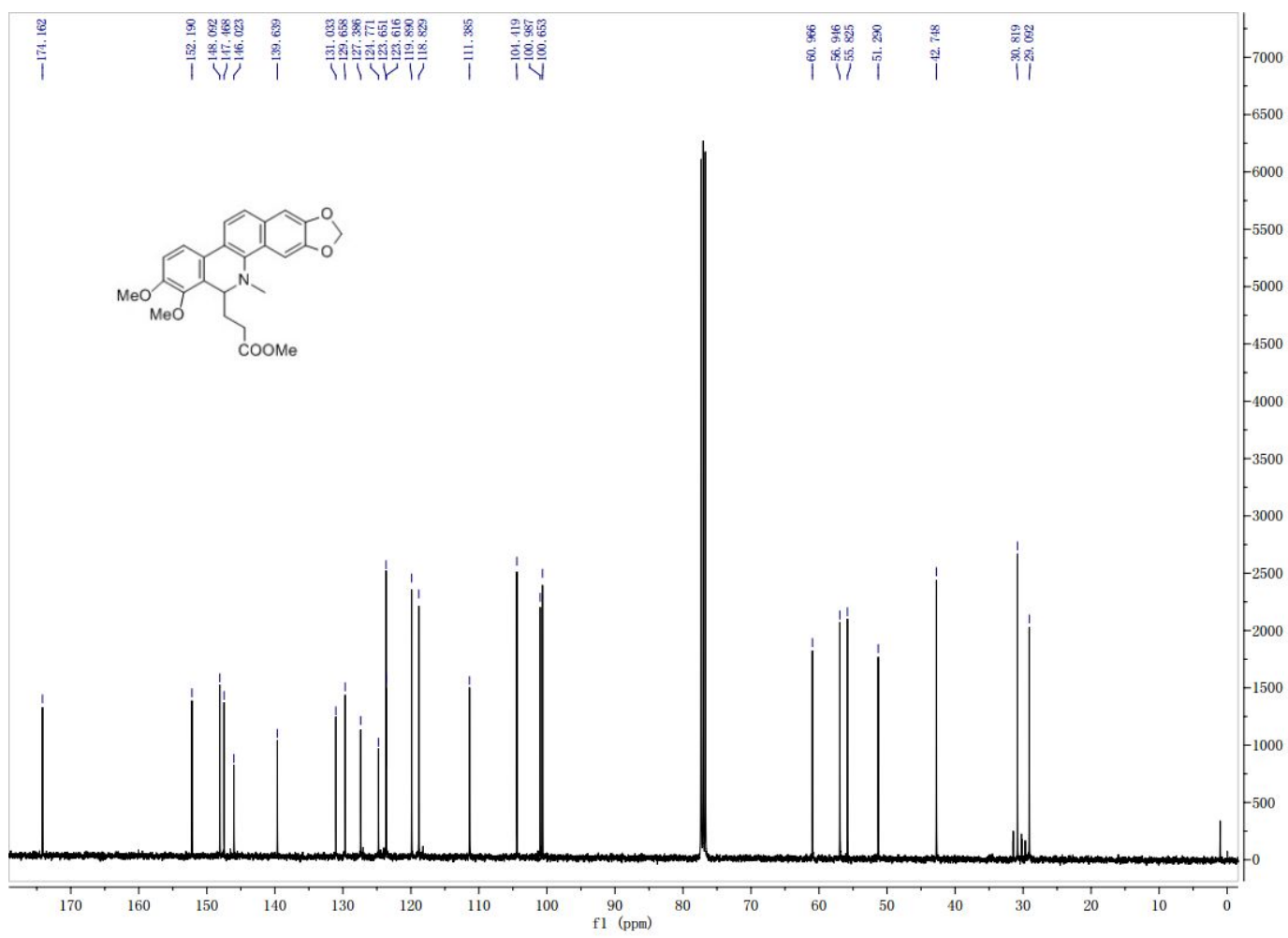

Figure S39. ${ }^{13} \mathrm{C}$ NMR spectrum $\left(400 \mathrm{MHz}, \mathrm{CDCl}_{3}\right)$ of 16aa

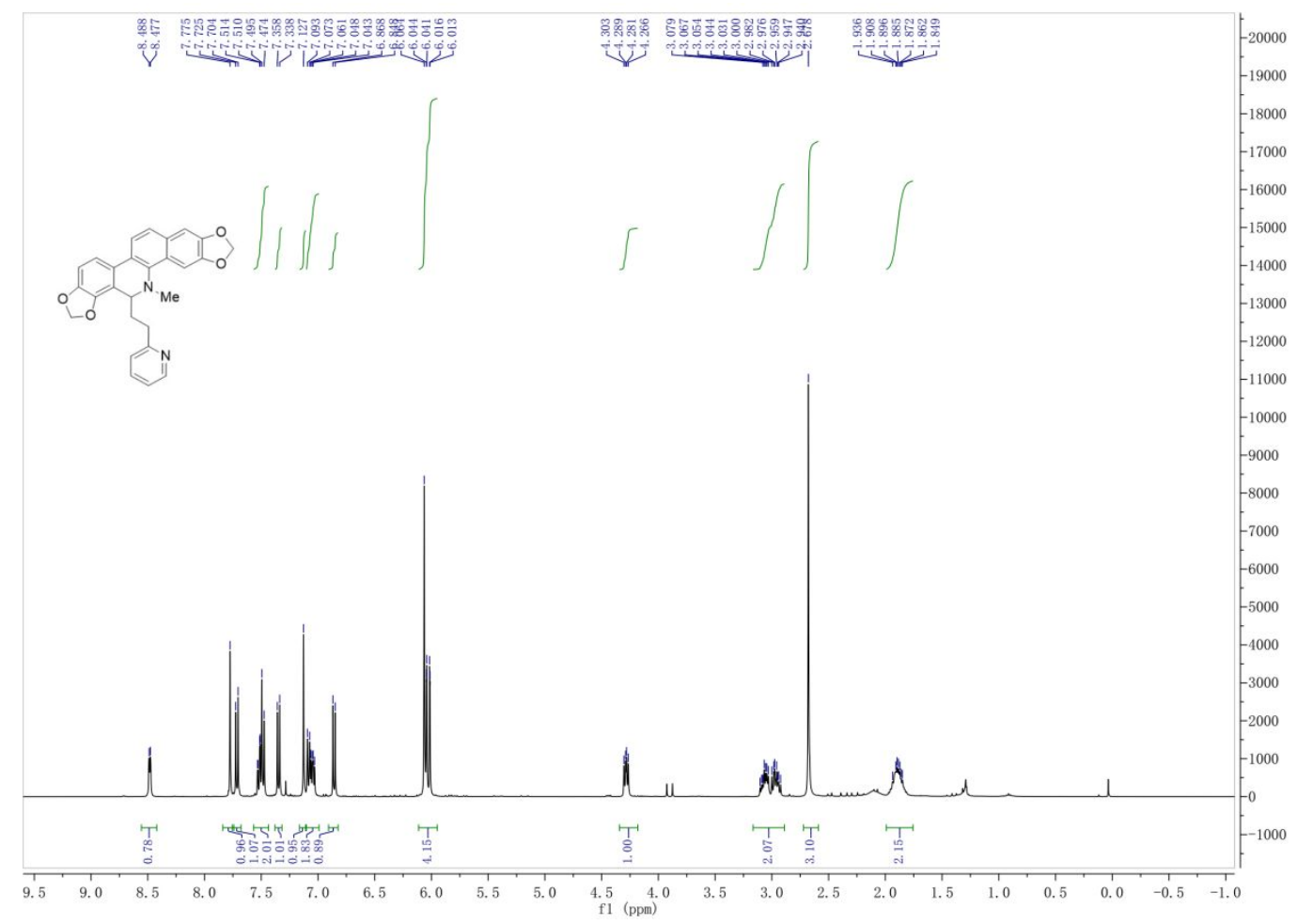

Figure S40. ${ }^{1} \mathrm{H}$ NMR spectrum $\left(400 \mathrm{MHz}, \mathrm{CDCl}_{3}\right)$ of $\mathbf{1 6 b}$ 


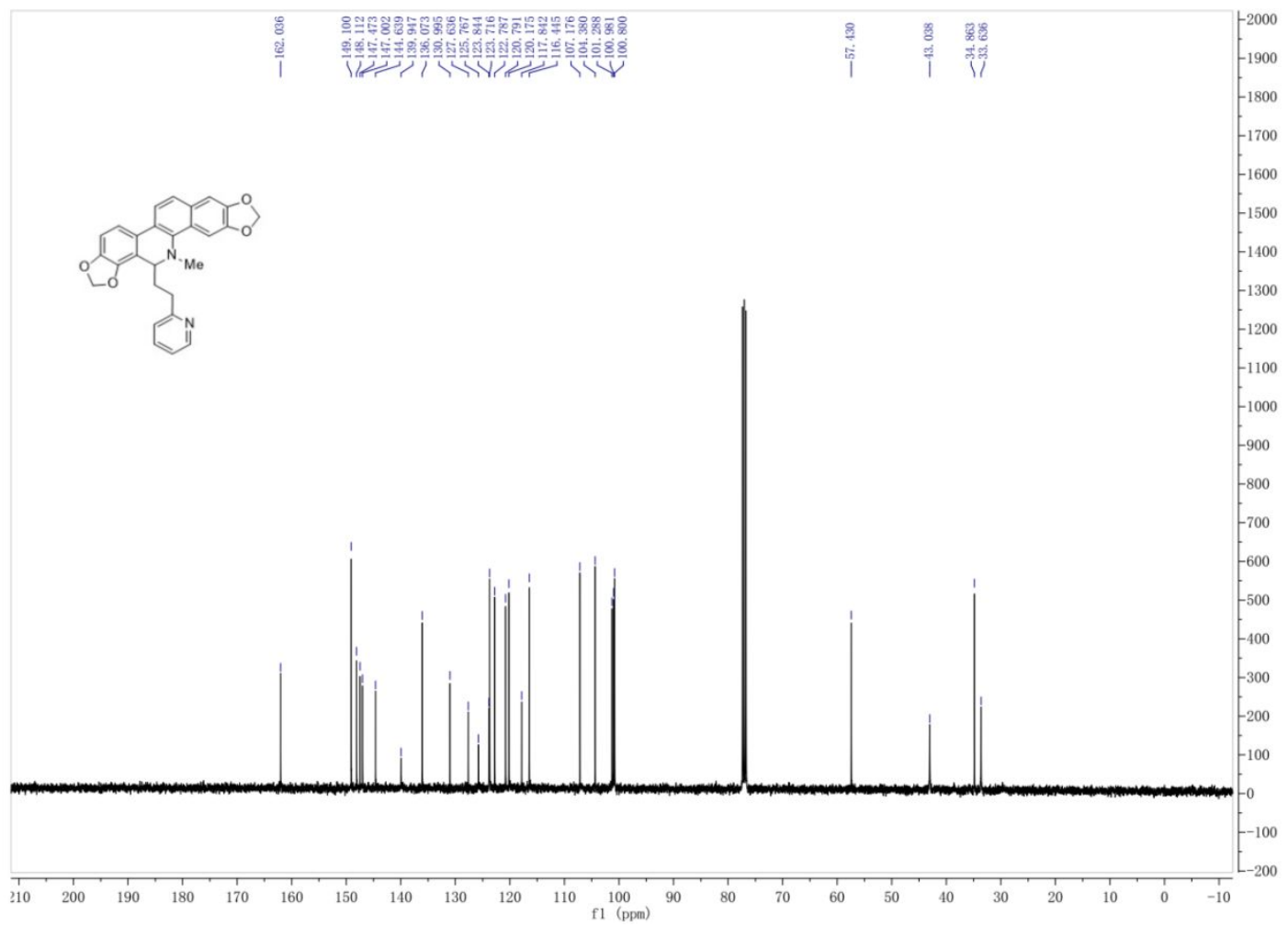

Figure S41. ${ }^{13} \mathrm{C}$ NMR spectrum $\left(400 \mathrm{MHz}, \mathrm{CDCl}_{3}\right)$ of $\mathbf{1 6 b}$

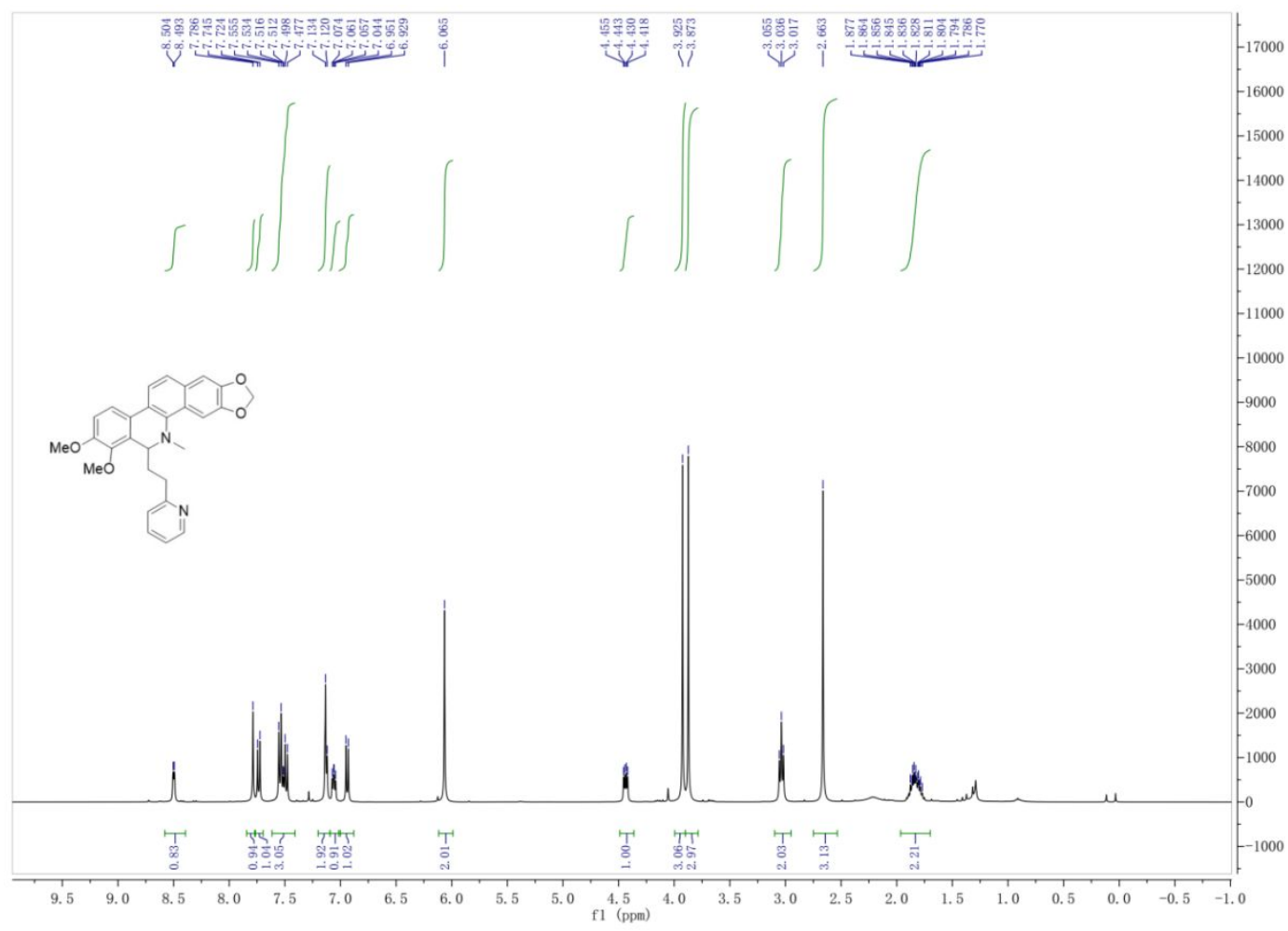

Figure S42. ${ }^{1} \mathrm{H}$ NMR spectrum $\left(400 \mathrm{MHz}, \mathrm{CDCl}_{3}\right)$ of $\mathbf{1 6 b b}$ 


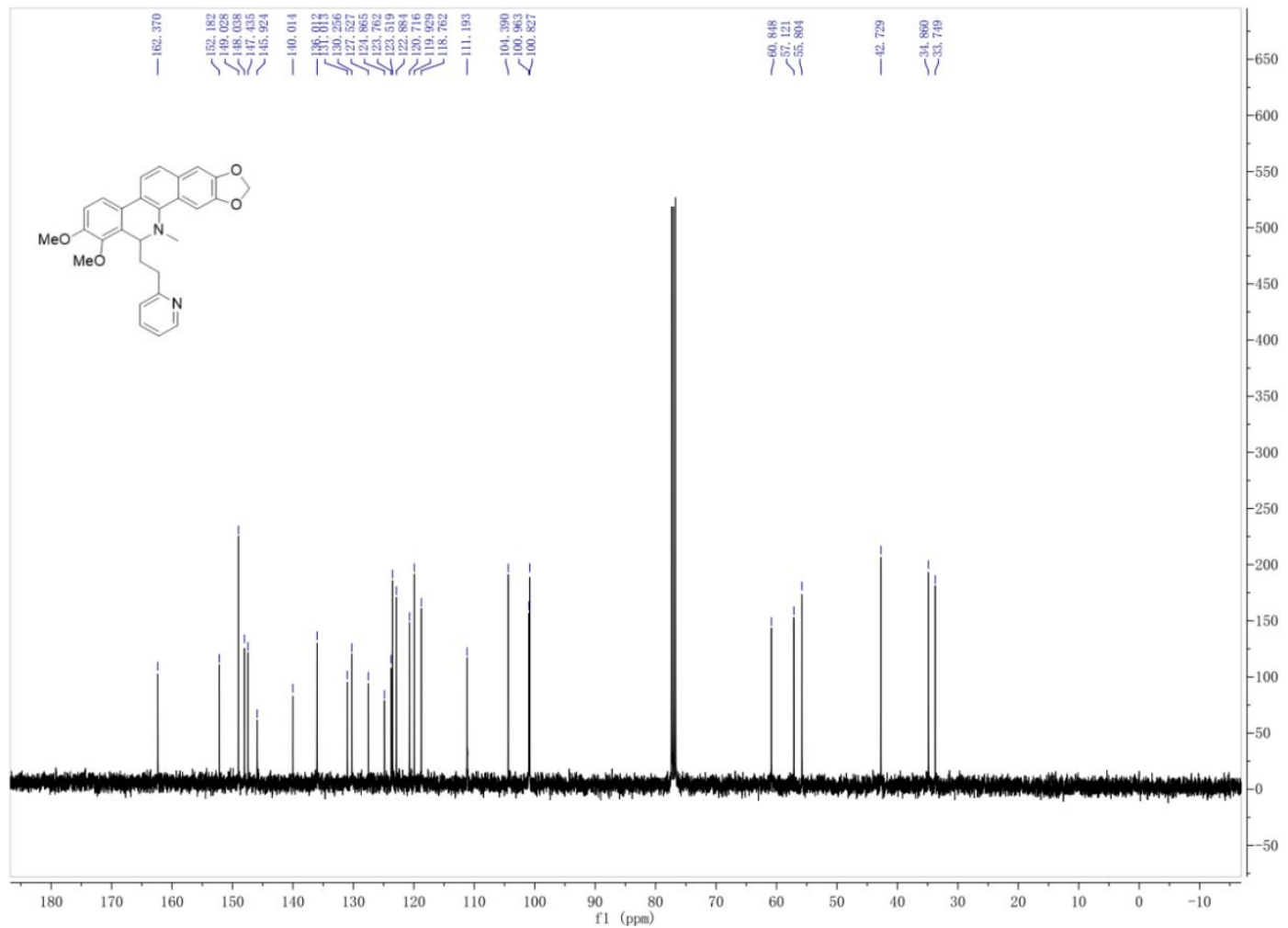

Figure $\mathbf{5 4 3} .{ }^{13} \mathrm{C}$ NMR spectrum $\left(400 \mathrm{MHz}, \mathrm{CDCl}_{3}\right)$ of $\mathbf{1 6 b b}$

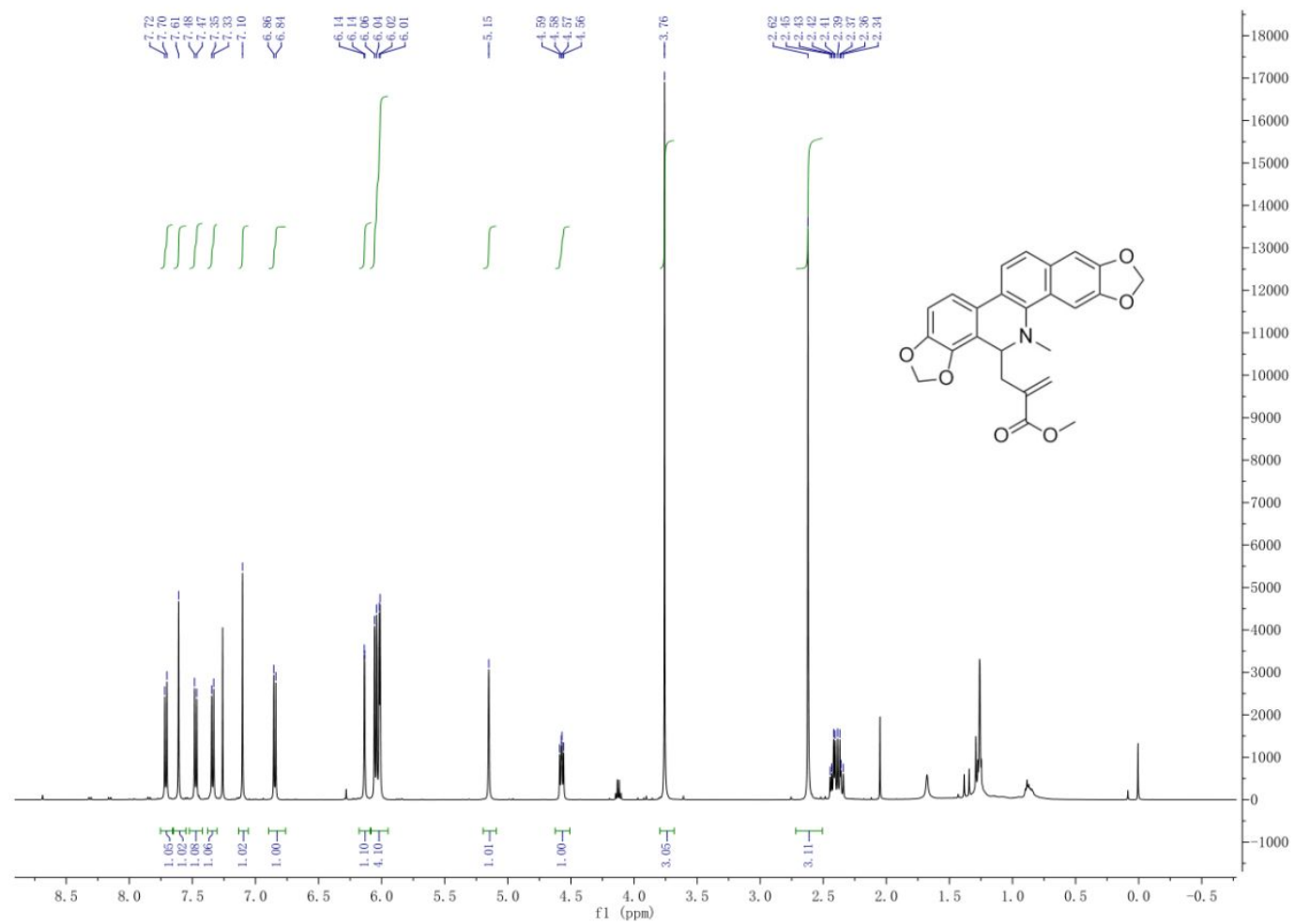

Figure S44. ${ }^{1} \mathrm{H}$ NMR spectrum $\left(400 \mathrm{MHz}, \mathrm{CDCl}_{3}\right)$ of $\mathbf{1 6 c}$ 


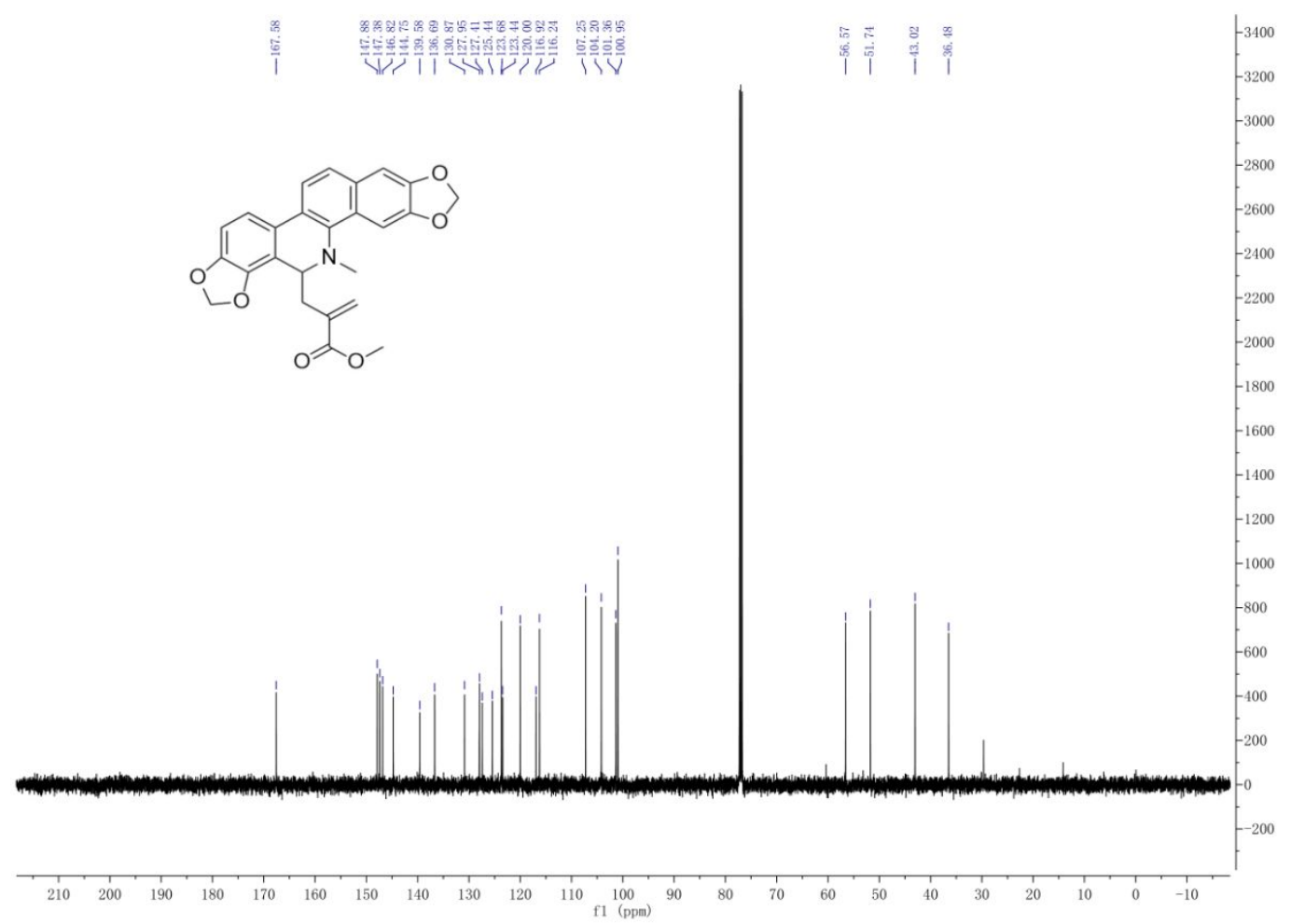

Figure S45. ${ }^{13} \mathrm{C}$ NMR spectrum $\left(400 \mathrm{MHz}, \mathrm{CDCl}_{3}\right)$ of $\mathbf{1 6 c}$

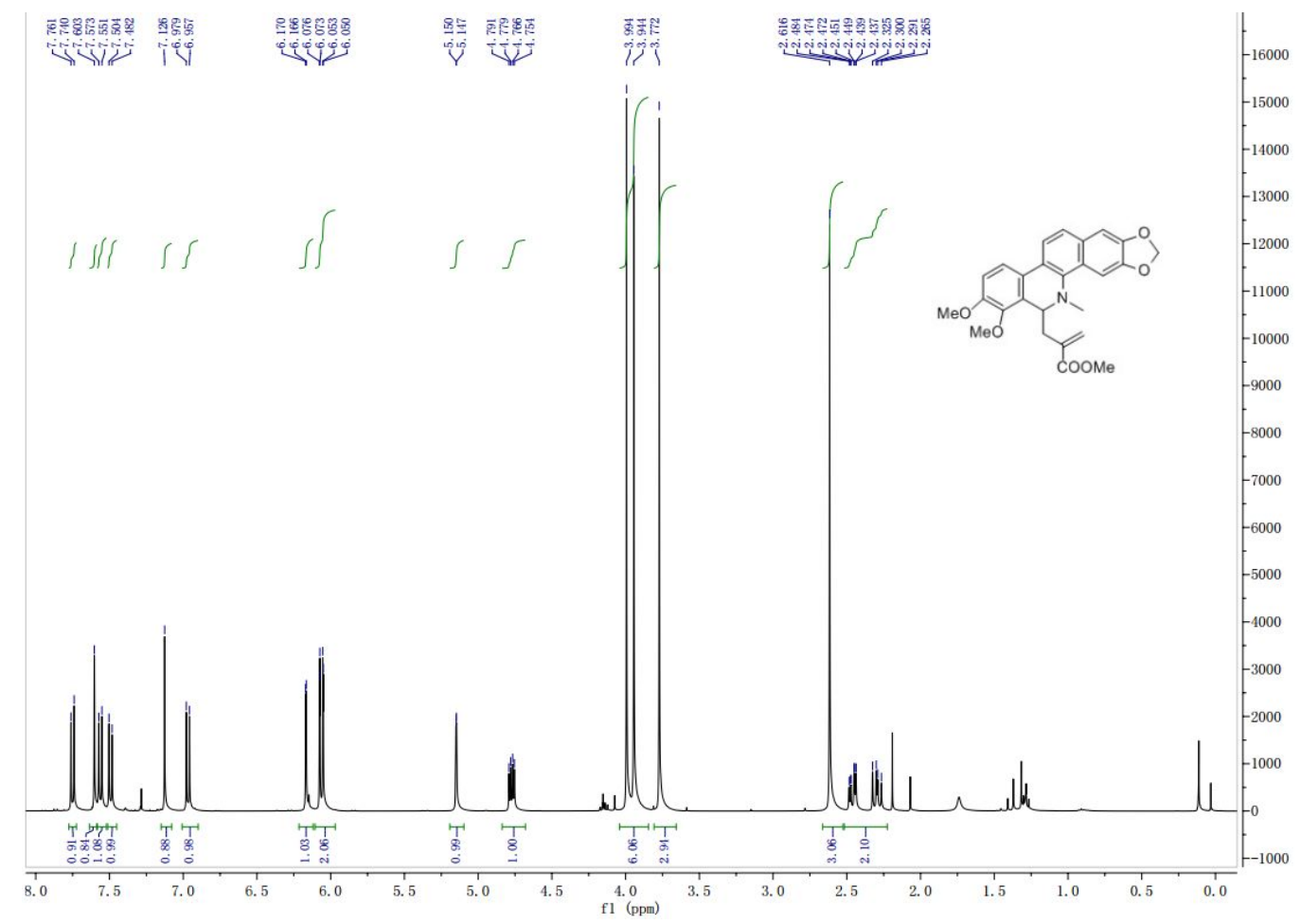

Figure S46. ${ }^{1} \mathrm{H}$ NMR spectrum $\left(400 \mathrm{MHz}, \mathrm{CDCl}_{3}\right)$ of $16 \mathrm{cc}$ 


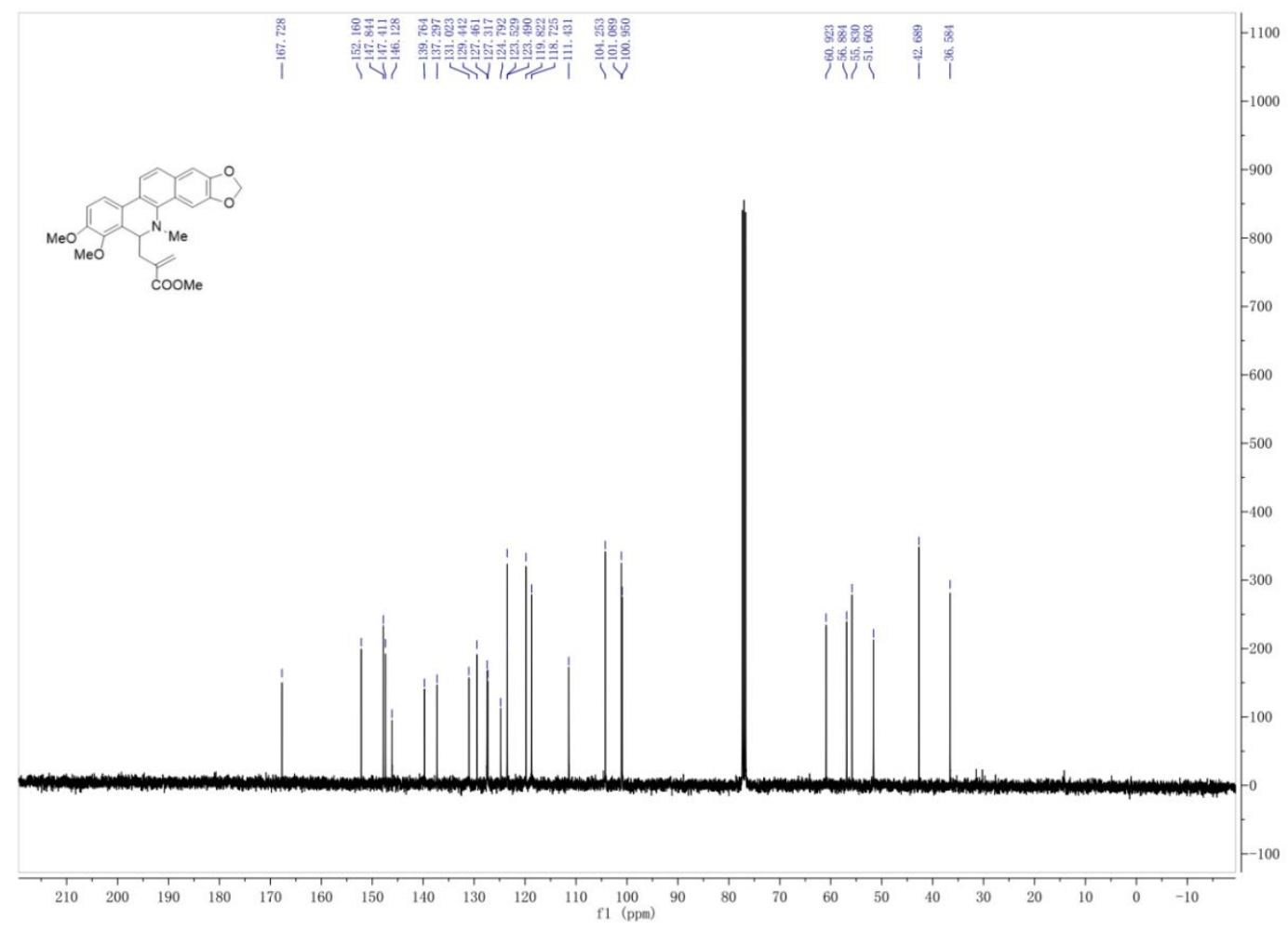

Figure $\mathbf{S 4 7} \cdot{ }^{13} \mathrm{C}$ NMR spectrum $\left(400 \mathrm{MHz}, \mathrm{CDCl}_{3}\right)$ of $\mathbf{1 6 c c}$

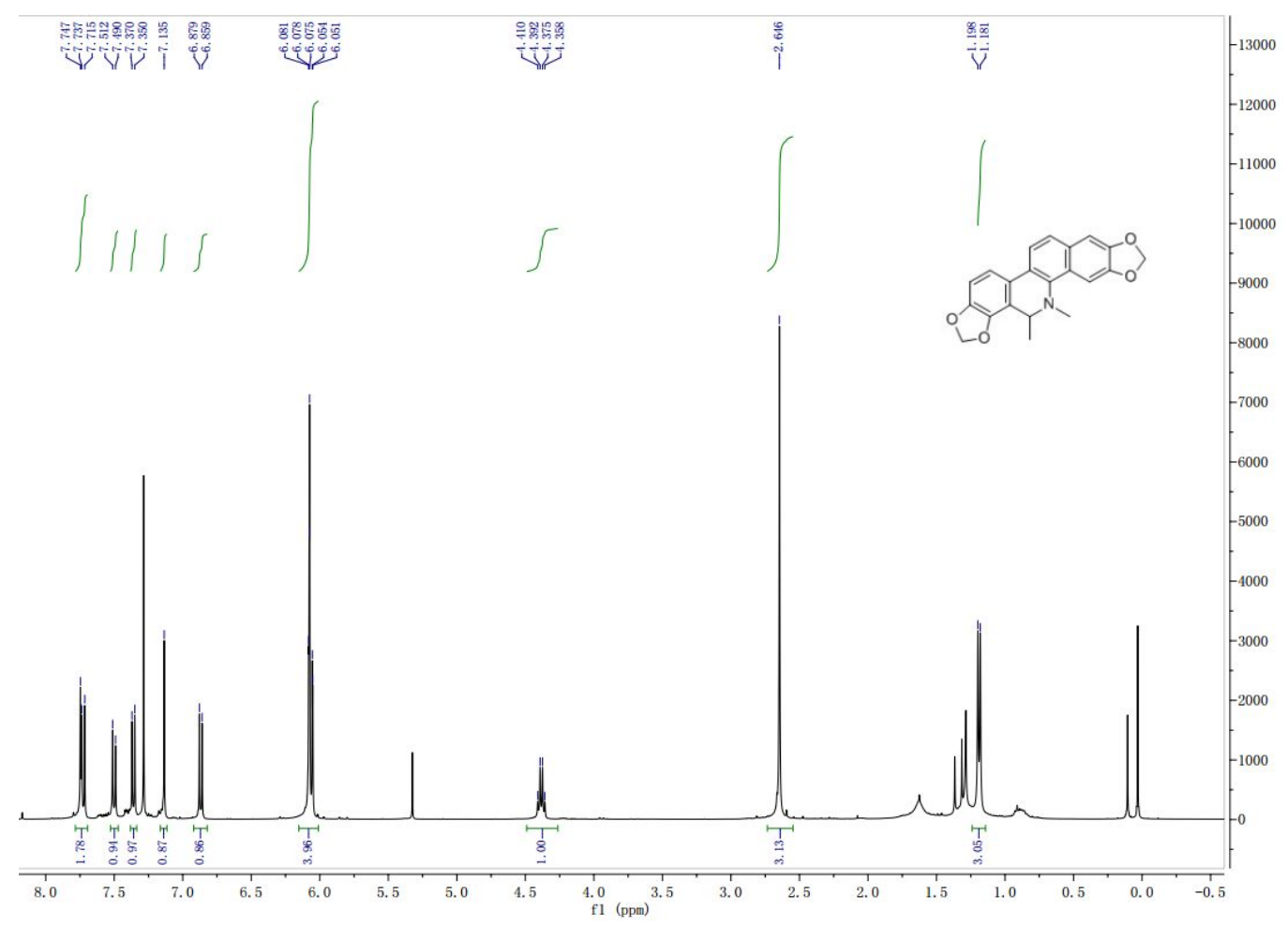

Figure S48. ${ }^{1} \mathrm{H}$ NMR spectrum $\left(400 \mathrm{MHz}, \mathrm{CDCl}_{3}\right)$ of 6-Me dihydrosauguinarine 


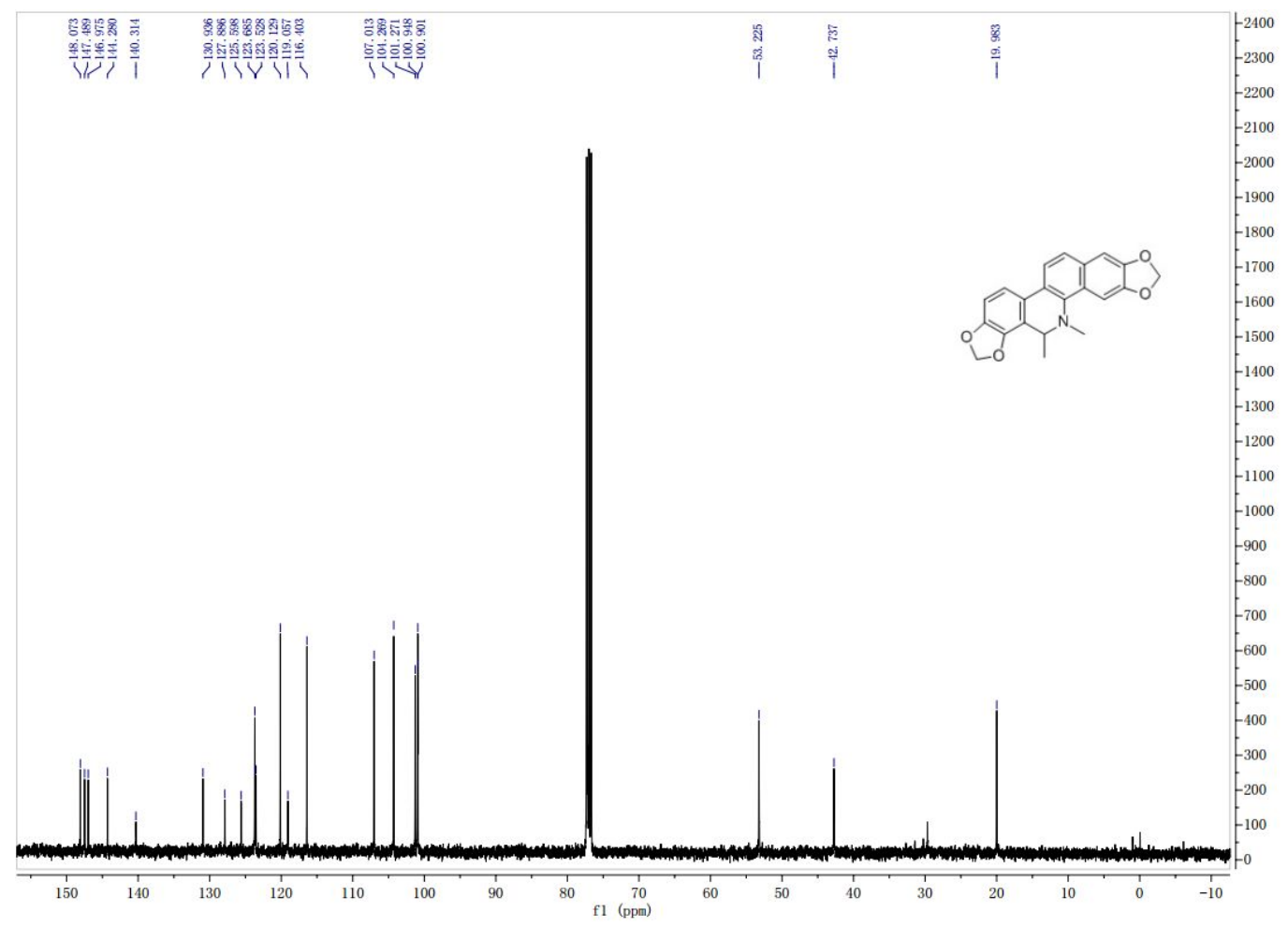

Figure S49. ${ }^{13} \mathrm{C}$ NMR spectrum $\left(400 \mathrm{MHz}, \mathrm{CDCl}_{3}\right)$ of 6-Me dihydrosauguinarine

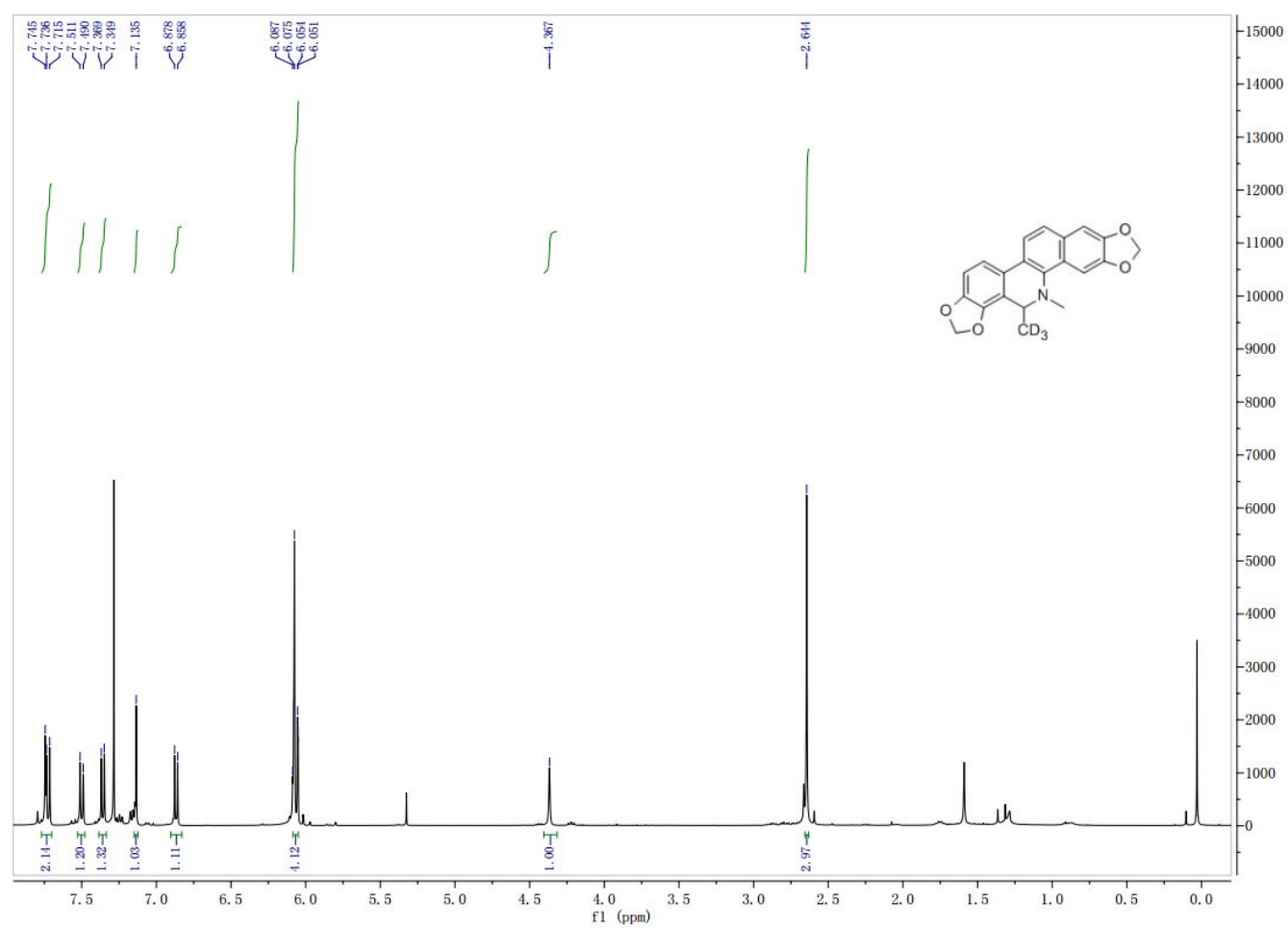

Figure S50. ${ }^{1} \mathrm{H}$ NMR spectrum $\left(400 \mathrm{MHz}, \mathrm{CDCl}_{3}\right.$ ) of $6-\mathrm{CD}_{3}$ dihydrosauguinarine 


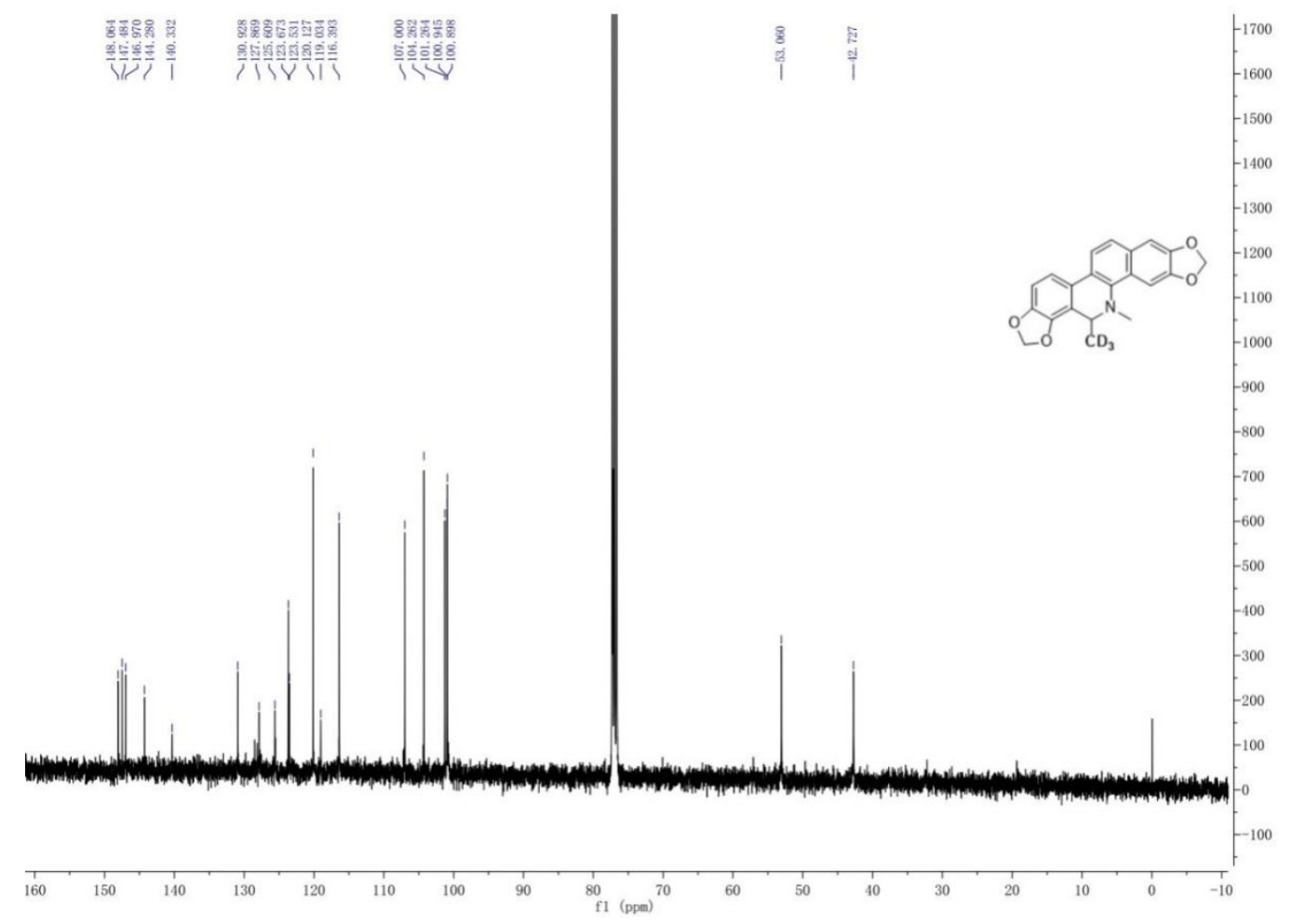

Figure S51. ${ }^{13} \mathrm{C}$ NMR spectrum $\left(400 \mathrm{MHz}, \mathrm{CDCl}_{3}\right)$ of $6-\mathrm{CD}_{3}$ dihydrosauguinarine

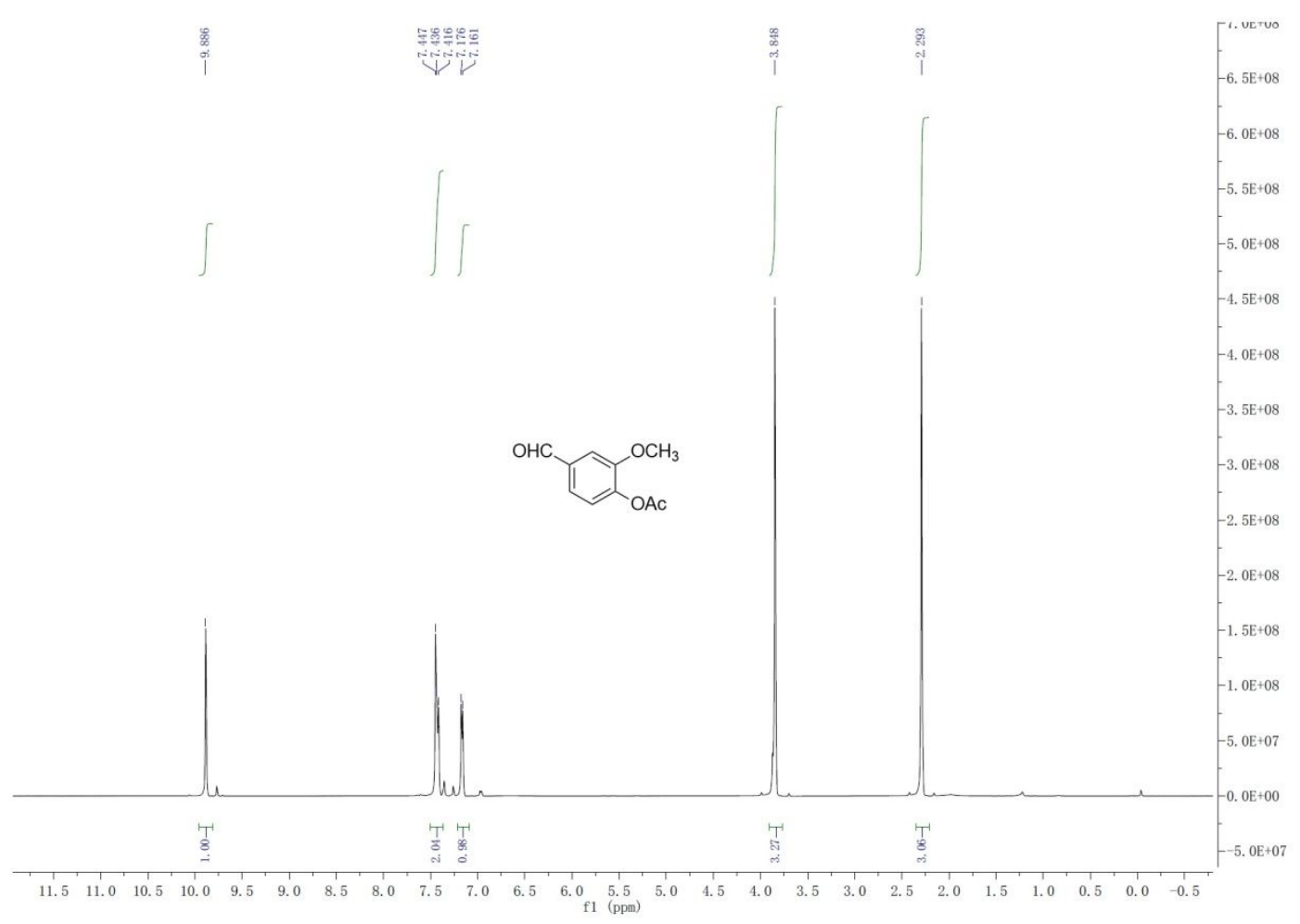

Figure S52. ${ }^{1} \mathrm{H}$ NMR spectrum $\left(400 \mathrm{MHz}, \mathrm{CDCl}_{3}\right)$ of 18 


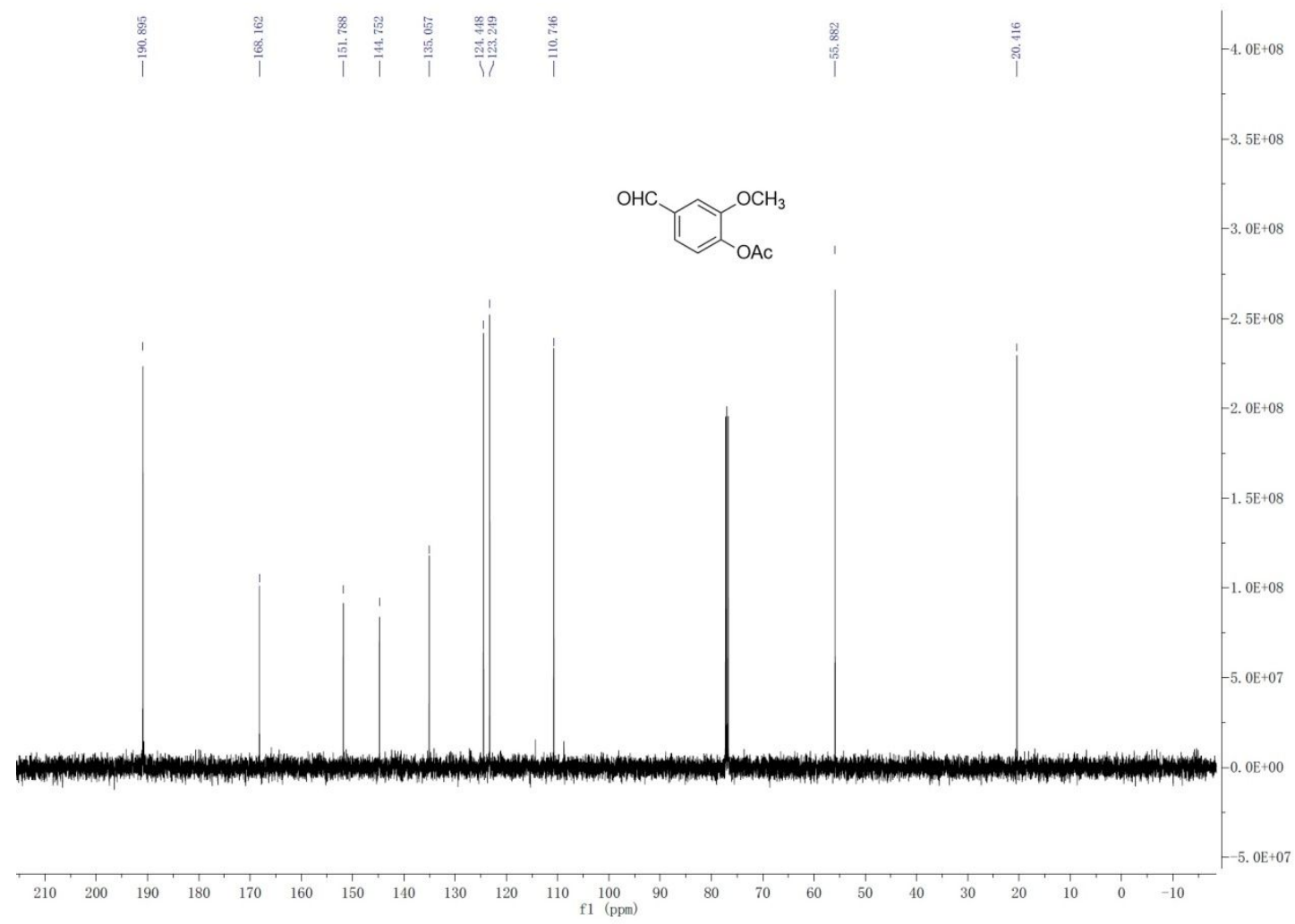

Figure S53. ${ }^{13} \mathrm{C}$ NMR spectrum $\left(400 \mathrm{MHz}, \mathrm{CDCl}_{3}\right)$ of $\mathbf{1 8}$

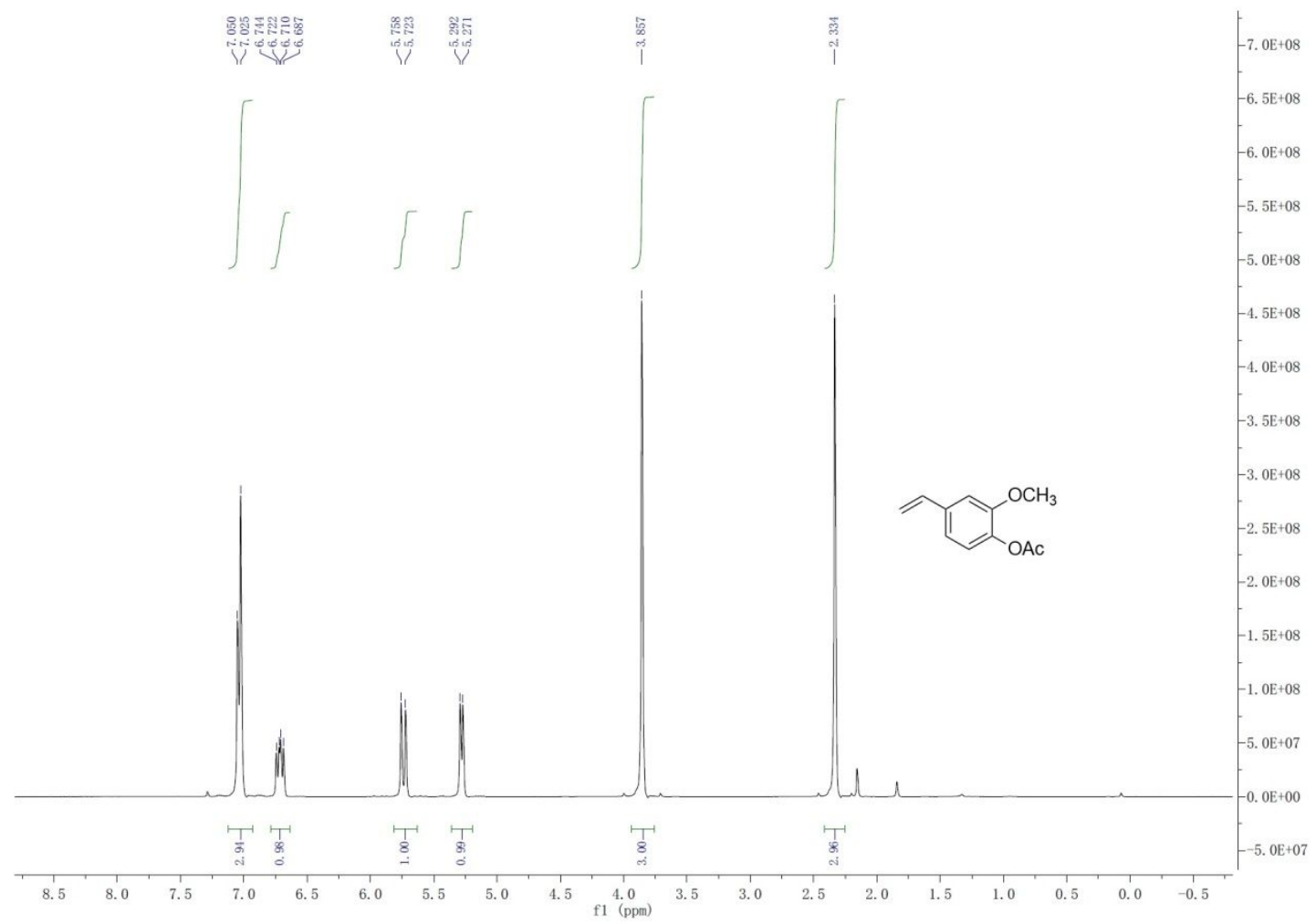


Figure S54. ${ }^{1} \mathrm{H}$ NMR spectrum $\left(400 \mathrm{MHz}, \mathrm{CDCl}_{3}\right)$ of 19

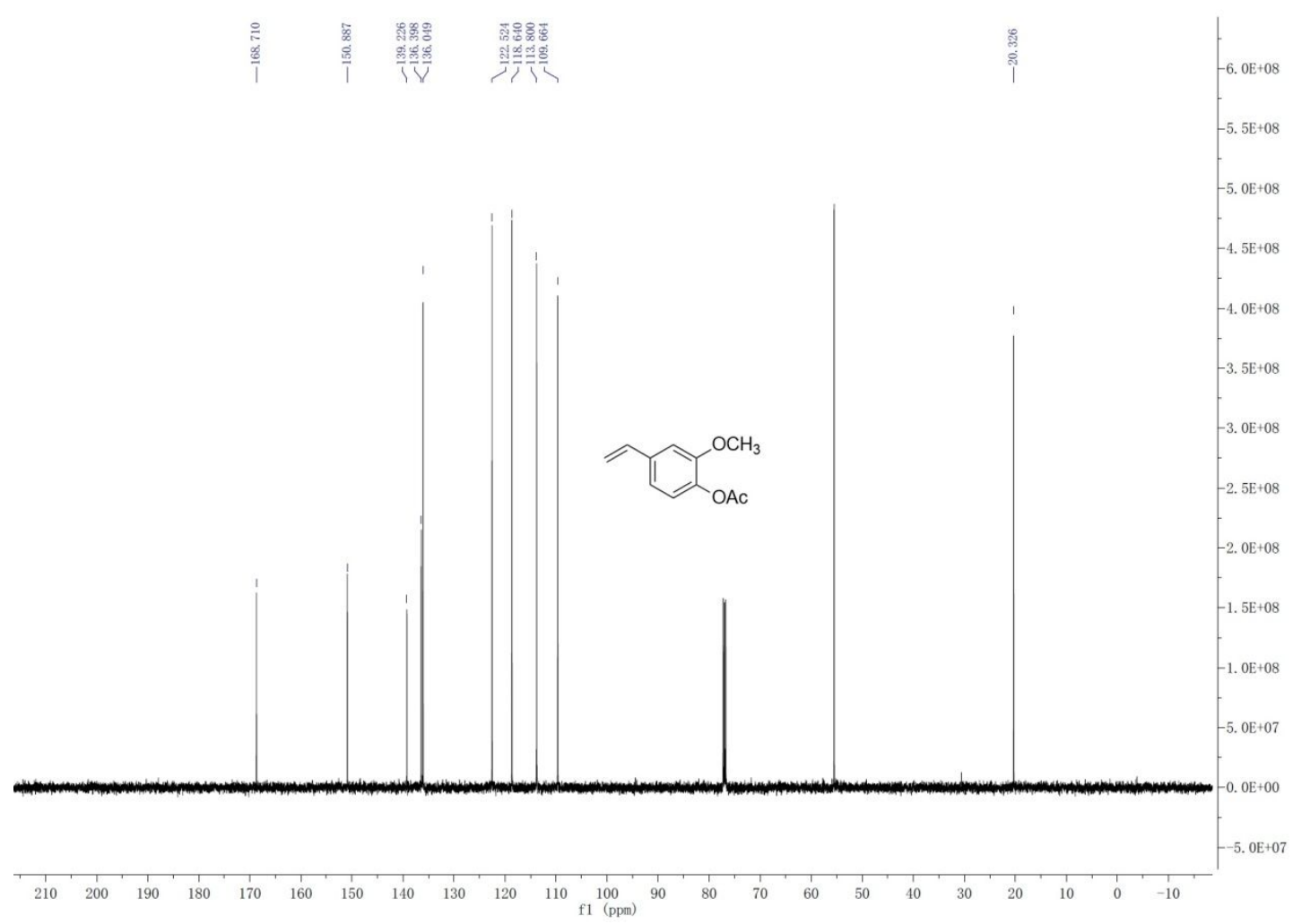

Figure S55. ${ }^{13} \mathrm{C}$ NMR spectrum $\left(400 \mathrm{MHz}, \mathrm{CDCl}_{3}\right)$ of 19

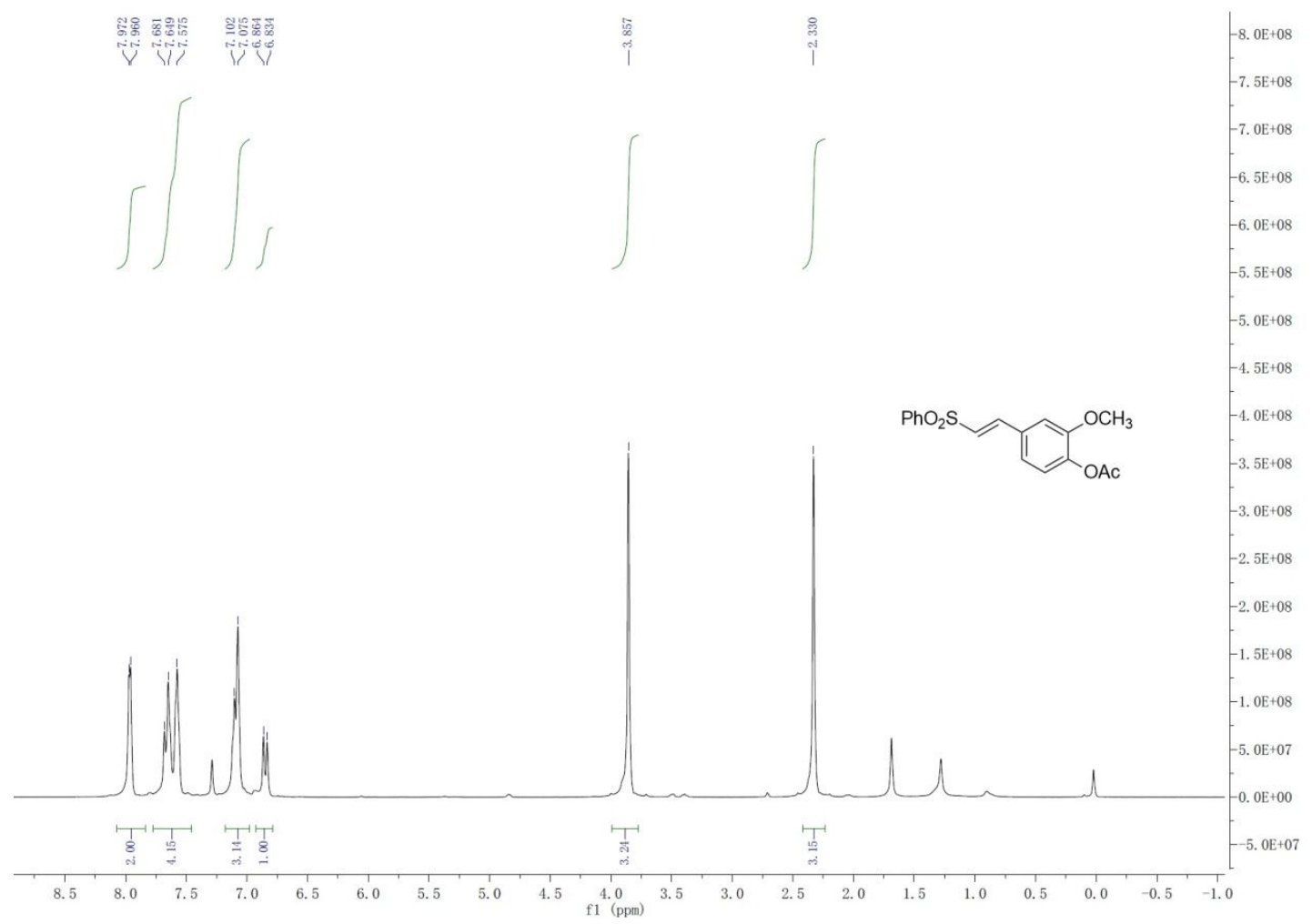

Figure S56. ${ }^{1} \mathrm{H}$ NMR spectrum $\left(400 \mathrm{MHz}, \mathrm{CDCl}_{3}\right)$ of 20 


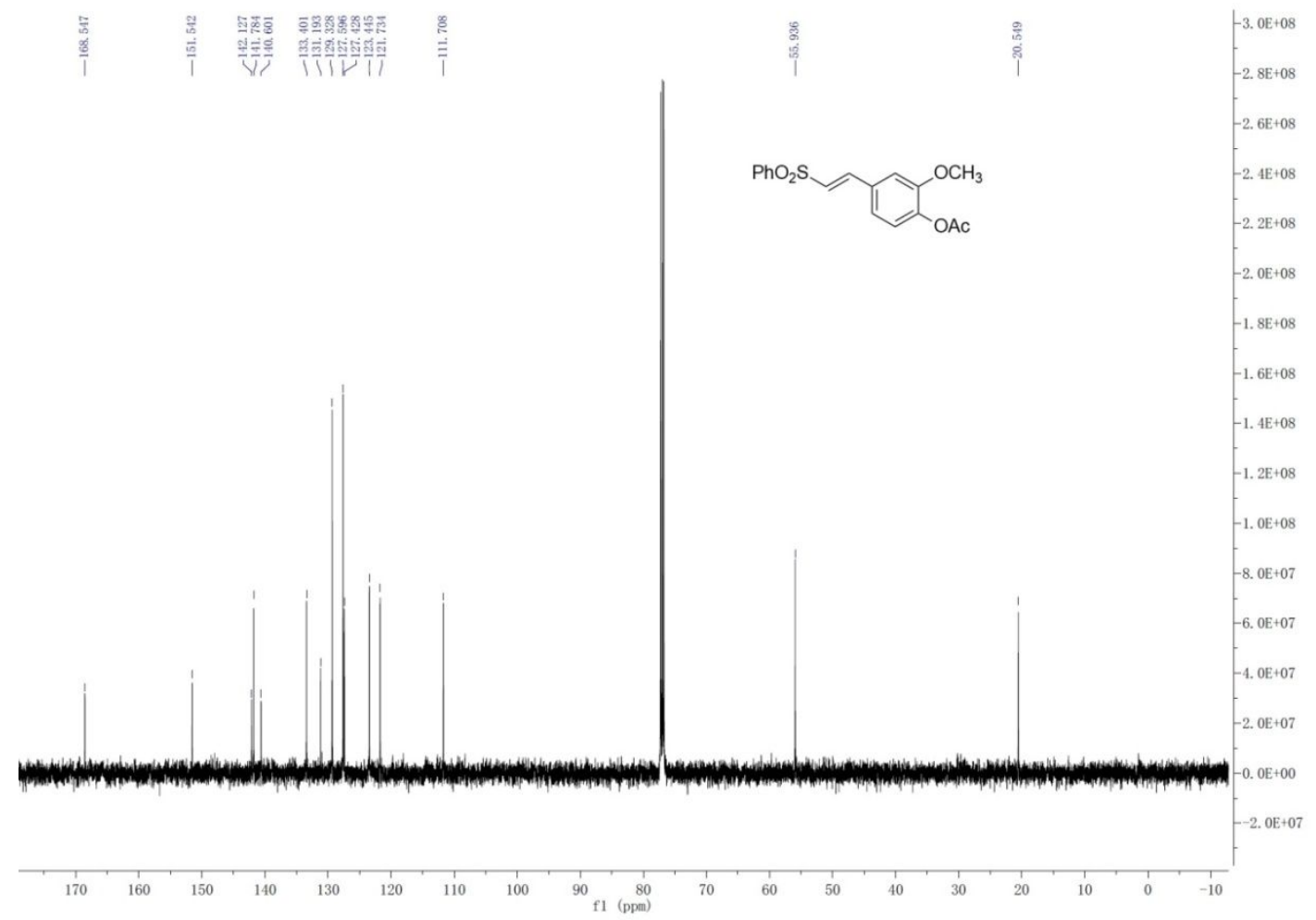

Figure S57. ${ }^{13} \mathrm{C}$ NMR spectrum $\left(400 \mathrm{MHz}, \mathrm{CDCl}_{3}\right)$ of 20

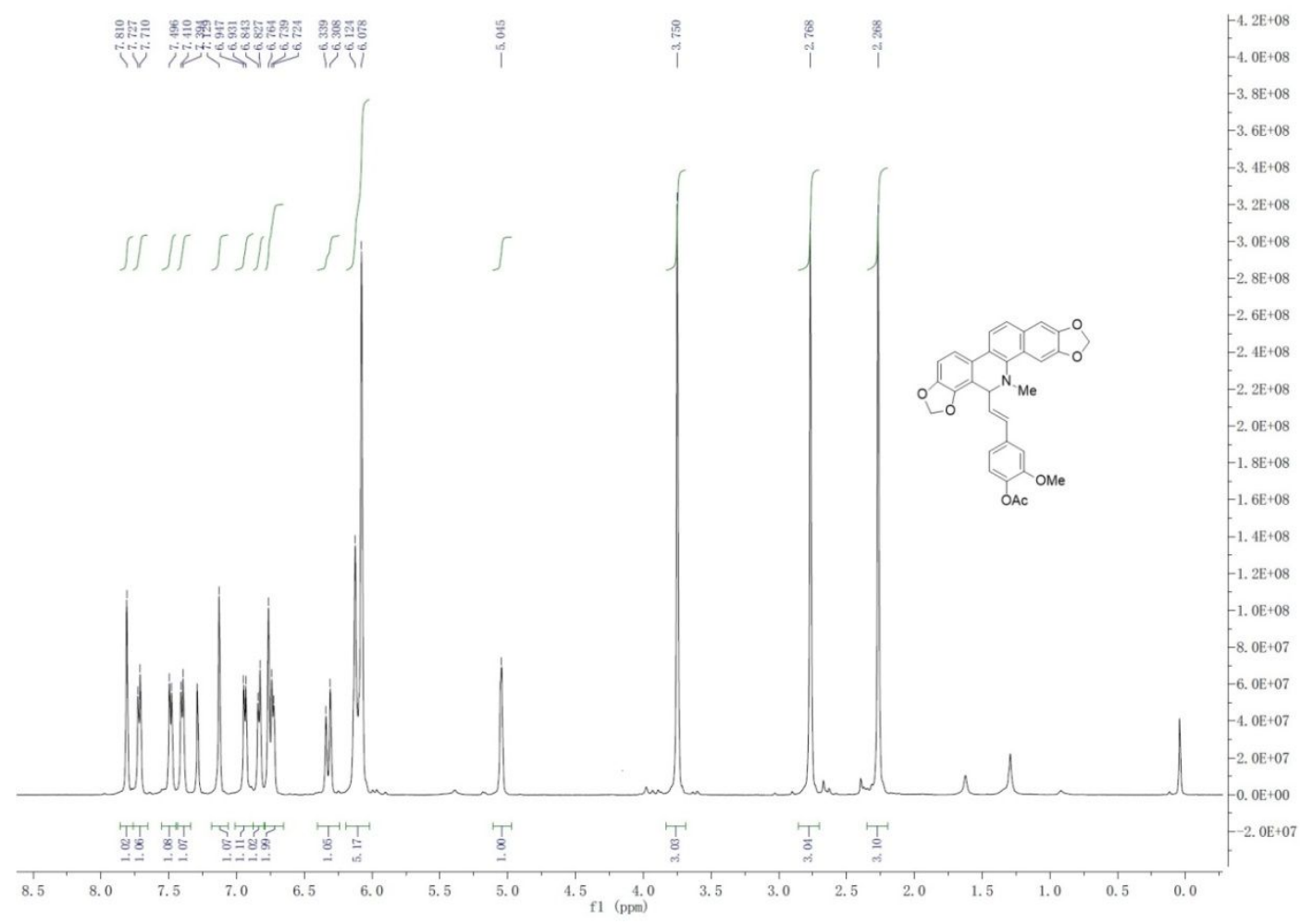

Figure S58. ${ }^{1} \mathrm{H}$ NMR spectrum $\left(400 \mathrm{MHz}, \mathrm{CDCl}_{3}\right)$ of 21 


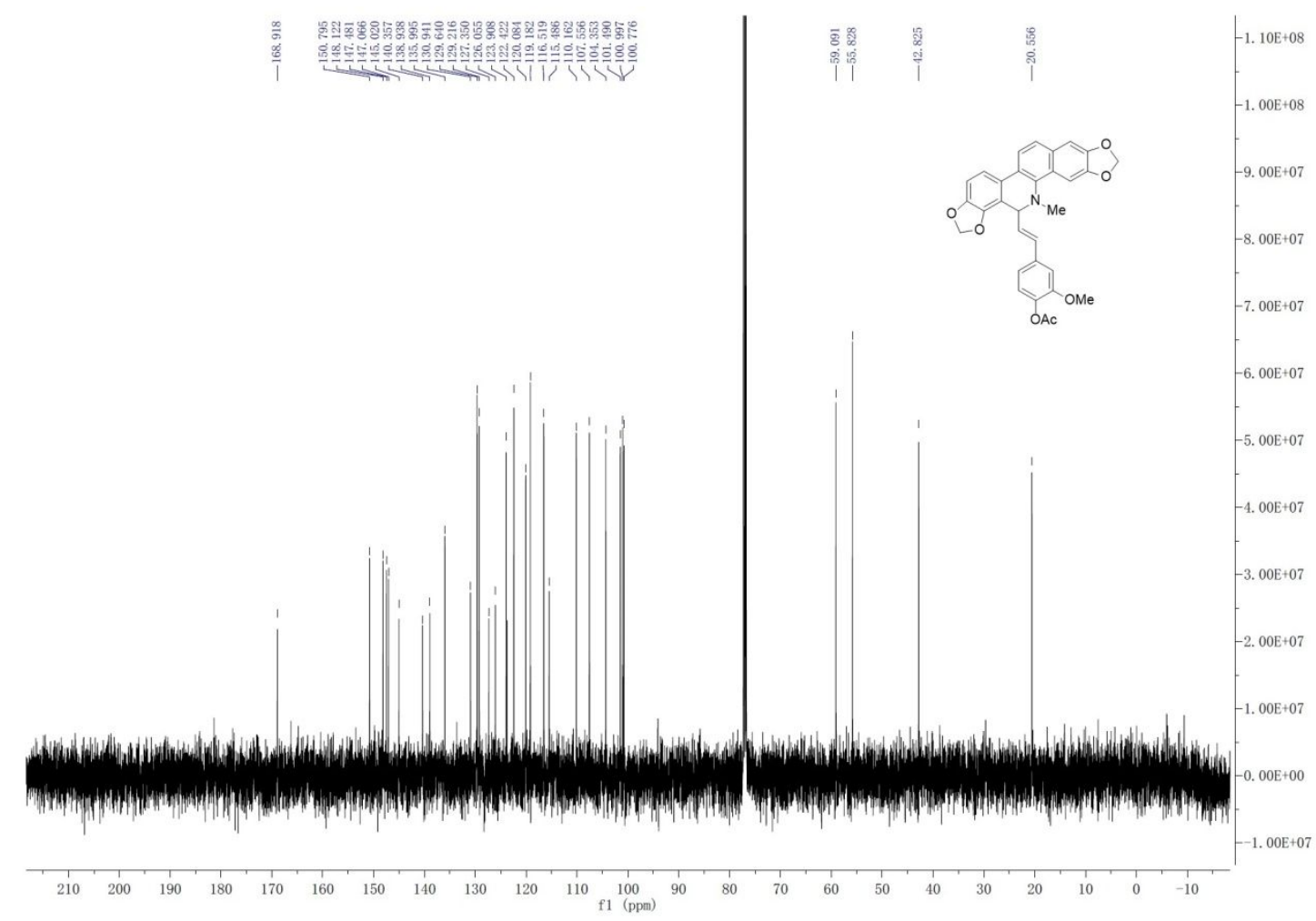

Figure S59. ${ }^{13} \mathrm{C}$ NMR spectrum $\left(400 \mathrm{MHz}, \mathrm{CDCl}_{3}\right)$ of 21 